XXV._Critical Experiments on the Chloroplatinate Method for the Determination of Potassium, Rubidium, and Ammonium; and a Redetermination of the Atomic Weight of Platinum. By W. Dittmar and John M'Arthur.

(Read 18th July 1887.)

The analytical methods referred to in our heading are infected with numerous sources of error, amongst which until lately the uncertainty of our knowledge of the combining constant " $\mathrm{Pt}$ " played a prominent part. This uncertainty, it is true, has been removed to a great extent by Seubert's investigation "Ueber das Atomgewicht des Platins," Liebig's Annalen (for 1881), vol. ccvii. p. 1 et seqq. The value $194.8 *$ for Pt, which he ultimately adopted, falls in well with his analyses of the chloroplatinates of potassium and ammonium, and there can be no doubt that his chloroplatinates were close approximations to the ideal substances. Hence his atomic weight 194.8 must be admitted to be nearer the truth than the value 198 of ANDREws, which, until lately, was so generally employed by chemists in the calculation of their analyses. But it does not follow that in, for instance, the determination of chloride of potassium "as metallic platinum," the factor $2 \mathrm{KCl}: \mathrm{Pt}=0 \cdot 7657$, calculated from SEUBERT's $\mathrm{Pt}$, gives a more correct result than even the factor 075252, which follows from $\mathrm{K}=39: \mathrm{Cl}=35.5$, and $\mathrm{Pt}=198$. The experience of practical analysts might almost be said to point the other way. A forcible illustration is afforded by Finkener's test-analyses in support of his own form of the chloroplatinate method for the determination of potassium. Finkener separates out the potassium as chloroplatinate (in admixture with the sulphates of the foreign metals present), and weighs it as platinum. In reducing his results, he used Andrews' value, $\mathrm{Pt}=198$ (or one not far removed from it), and obtained close approximations to his syntheses. Had he calculated with SeuberT's number, 194.8, his results, if reduced to $\mathrm{K}_{2} \mathrm{O}$, would have been too high by nearly 2 per cent. All this, of course, goes no hair's-breadth towards invalidating SEUBERT's result; it only shows that those analytical factors which are, by theory, equal to $\mathrm{K}_{2}: \mathrm{Pt}$; $\mathrm{K}_{2}: \mathrm{PtCl}_{6} \mathrm{~K}_{2}$, \&c., must be determined directly by standard experiments; and separately for the several methods. This is what we have attempted (in a limited sense) to do. But a purely empirical determination of these factors by even the most exact experiments would have been of little use. If, for

* Let us state at once that in this memoir all atomic weights are referred to $O=16$. For the atomic weights of potassium, ammonium, and chlorine we adopt STAs' numbers, as calculated by LotUaR Meyer and Seubert; $\mathrm{K}=39 \cdot 136 ; \mathrm{NH}_{4}=18 \cdot 056 ; \mathrm{Cl}=35 \cdot 455$.

VOL. XXXIII. PART II. 
instance, the analyst's factor for $\mathrm{K}_{2}$ : Pt differs from the ratio $2 \times 39 \cdot 136: 194 \cdot 8$, the causes of this difference must be ascertained, and this necessarily involves an inquiry into the true value for " $\mathrm{Pt}$ "; STAs' numbers for $\mathrm{KCl}$, \&c., can, of course, be taken for granted. We accordingly did take up this inquiry, and in this sense our investigation joins on to SEuberT's, which, of course, was welcome to us, as affording a most valuable basis for this branch of our work.

Before proceeding to detail our experiments, let us state that, unlike our predecessors, we did not merely analyse our chloroplatinates, but in their productions used exactly known quantities of standard solutions of chloroplatinic acid and (for instance) chloride of potassium; so that the weights of platinum and alkaline chloride contained in the precipitated chloroplatinate could be ascertained by determining the small quantities of these which passed into the mother-liquor, and deducting them from the weights of platinum and chloride of potassium employed in the precipitation. In the preparation and application of these standard solutions (and of standard solutions generally) we used a system which was introduced some years ago by one of us in connection with a series of analyses of ocean water,* and which consists in this, that the solutions are standardised both by volume and by weight. The volume-titre serves only for calculating the number of cubic centimetres of solution containing a predetermined quantity of reagent: the exact weight of the latter is calculated from the weight of the measured off quantum and the weight-titre.

The necessary special reagents were prepared in the following manner :-

\section{Chloroplatinic Acid.}

For the preparation of this reagent, we utilised two supplies of what had been sold to us as "pure platinum." One of these came from the St Petersburg Works, through the kind agency of a St Petersburg gentleman, who happened to be in Glasgow at the time; the other, from Messis Johnson, Matthey, \& Co. of London. The St Petersburg metal, when examined, turned out to be contaminated with iridium so largely that we presume a mistake must have been made in the transmission or execution of our order. To purify the metal we dissolved it in aqua regia, as far as possible, $t^{+}$and subjected the crude chloroplatinic acid thus produced to the process of SchneIder, which is so fully described by SeuberT in his memoir. The pure chloroplatinate of ammonium obtained was recluced to metal by ignition, the platinum washed with hydrochloric acid, then with water, dissolved in aqua

* "Challenger Reports," Physics and Chemistry, vol. i., published by order of Her Majesty's Stationery Office, \&c. Adam and Charles Black (and others).

$\dagger \mathrm{A}$ good deal of black iridium remained. 
regia, and eliminated from the solution by means of pure hydrogen in the wet way. The metallic platinum was then filtered off, was washed successively with hot water, hot hydrochloric acid, and again with water, dried, and ignited in a porcelain crucible. When 23.338 grms. of this metal were dissolved in dilute aqua regia, with the view of preparing a standard solution, a black, sooty-looking residue remained, which was removed by filtration, ignited, and weighed. It amounted to $25 \cdot 7 \mathrm{mgs}$. The solution was freed from nitric acid by repeated evaporation on a water-bath with hydrochloric acid, the ultimate residue dissolved in water, diluted to 471 c.c. in a tared bottle, and the solution weighed. It weighed 509.969 grammes, and, taking the part soluble in aqua regia as pure platinum, contained 23.3164 grms. of this metal. For roughly quantitative work, it was assumed to contain $49.5 \mathrm{mgs}$. of platinum $=\frac{1}{4} \times 198$ mgs. per c.c.

Strictly speaking, we had no right to assume that all the metal contained in this solution was pure platinum; but as the solution exhibited a normal colour, and gave light yellow precipitates with chlorides of potassium and ammonium, we thought it might safely be used as pure chloroplatinic acid of the above titre, the more so as the atomic weight of iridium is only a little less than that of platinum, and a small admixture of the former metal in a chloroplatinate could not appreciably affect the percentage of metal in it. We accordingly did use this solution in some of our experiments.

Matthey's Platinum.- To examine this metal for impurities, 10 grms. of it were dissolved in hydrochloric acid, with the aid of the least sufficiency of nitric. The solution was very light in colour, and no visible residue remained. The solution was transferred to a large platinum basin, mixed with a large excess of caustic soda,* boiled for 20 minutes, the hypochlorite destroyed by alcohol, and the resulting liquid acidified strongly with hydrochloric acid. No permanent precipitate was produced. The solution was precipitated with sal-ammoniac, the chloroplatinate (which was light yellow) filtered off, and the filtrate concentrated to a small volume, and allowed to stand to recover a second instalment of chloroplatinate. The filtrate from this was evaporated to dryness, the sal-ammoniac burned off, the residue washed with water, ignited, and weighed. It weighed $123 \mathrm{mgs}$. On treatment with aqua regia, a white residue (silica ?) remained, which weighed $3 \cdot \cdot 6 \mathrm{mgs}$. after ignition. Hence metal in solution $=90.4 \mathrm{mgs}$. This solution was mixed with a weighed quantity of a standard solution of chloride of potassium, containing $64.79 \mathrm{mgs}$. of $\mathrm{KCl}$ (corresponding to about 0.95 time $90.4 \mathrm{mgs}$. of platinum), the mixture evaporated to a very small volume, and mixed, after cooling, with absolute alcohol, to precipitate the "chloroplatinate" produced. The precipitate, after

* Specially made from pure (Trommsdorff's) carbonate-crystals, in a nickel basin. 
drying at $110^{\circ} \mathrm{C}$., weighed $217.9 \mathrm{mgs}$.; $193.5 \mathrm{mgs}$. of it, when reduced in hydrogen (dry-way), yielded $77.8 \mathrm{mgs}$. of metal, or $87.61 \mathrm{mgs}$. per $217.9 \mathrm{mgs}$. of precipitate. The filtrate from the "chloroplatinate" contained $2.4 \mathrm{mgs}$. of "platinum," and $0.49 \mathrm{mg}$. of chloride of potassium (determined and weighed as $\mathrm{PtCl}_{6} \mathrm{~K}_{2}$ ). Hence chloride of potassium in the precipitate $=64.79$ (weight of $\mathrm{KCl}$ used) $-0.49=64.3 \mathrm{mgs}$. Platinum (1) by synthesis, $=88 \cdot 0$; (2) by analysis, $=87.61$; hence platinum per $2 \mathrm{KCl}$ by $(1)=2042 ;$ by $(2)=203.3$. These numbers are certainly higher than the true atomic weight of platinum, but, considering the small scale on which the analysis was made, and that all the impurities of the $10 \mathrm{grms}$. of original metal were concentrated in that small remnant of $90.4 \mathrm{mgs}$. of metal, the original metal, we thought, could be accepted as sufficiently pure. To prepare a standard solution, 49.5327 grms. of Matthey's metal (sponge) were ignited in a porcelain crucible, which brought down the weight to 49.5302 grms. These were dissolved in a Berlin basin, under a funnel, in aqua regia, made from specially prepared acids, the nitric acid expelled by repeated evaporation with hydrochloric acid, the ultimate residue dissolved in water, diluted to 1 litre, and weighed. A small quantity of an insoluble residue separated out on dissolving the chloroplatinic acid crystals in water ; it was filtered off, ignited, and weighed, and its weight, $3.4 \mathrm{mgs}$., deducted from the original weight of platinum taken, as a correction. The solution was preserved as containing very nearly $49 . \tilde{\mathrm{mgs}} \mathrm{m}$. of metal per c.c., and exactly $0.4 \tilde{0} 930 \mathrm{grm}$. per 10 grammes of solution.

The vast majority of our quantative syntheses and analyses were made with this or some other standard solution derived from Matthey's metal. Wherever St Petersburg metal was used, this is specially stated.

Chloroplatinic acid, as prepared by means of the aqua regia process, is liable to be contaminated with the nitroso-compound $\mathrm{PtCl}_{6}(\mathrm{NO})_{2}$. After having made a considerable number of syntheses and analyses of alkaline chloroplatinates, we found that one of our solutions at least was thus contaminated. It is difficult to see how a small admixture of the nitroso-body could affect a synthesis of potassium or rubidium chloroplatinate, yet its presence is at best no improvement; hence, after that discovery, we tried to make platinum solution by means of hydrochloric acid and chlorine gas, i.e., the method which Seubert used once (for his Darstellung III., p. 16 of his memoir), to return to the old process. We soon saw how it came that SEubert tried the process only once; the solution of the metal takes place with an exasperating degree of sluggishness.

In the following form, however, we found the method to work satisfactorily. The platinum, preferably in the spongy form, is introduced into a light-coloured glass-stoppered bottle of, say, 2 litres' capacity; a sufficiency of fuming hydrochloric acid is poured on it, and the bottle filled with chlorine 
CRITICAL EXPERIMENTS FOR DETERMINATION OF POTASSIUM, ETC. 565

gas (purified by washing with water, and filtration through asbestos), the stopper put on, and the whole allowed to stand.

After about twelve hours the chlorine is mostly absorbed. The bottle is then refilled with chlorine, again allowed to stand, \&c., until the metal is all, or mostly, dissolved. The solution is decanted off, again chlorinated to make sure of the absence of platinous salt, evaporated on a water-bath to expel the surplus chlorine and hydrochloric acid, and diluted to the proper volume. Of course, if it is meant to be used as an exact standard solution, the remnant of undissolved metal is collected, washed, ignited, weighed, and allowed for. With a "chlorine Kipp" (see DitTmar's Exercises in Quantitative Analysis, p. 137) at hand, the method is not so tedious as it appears at first sight. We have long come to adopt it even for ordinary laboratory purposes. When used in connection with work here reported on, it is referred to as the "chlorine process."

\section{Chloride of Potassium.}

A few of our earlier experiments were made with a chloride obtained from recrystallised chlorate, but in the majority of cases we prepared our chloride of potassium from recrystallised perchlorate. The perchlorate is heated in a platinum basin until the bulk of the oxygen is removed. The rest is then expelled by fusing the residue in a platinum crucible, and keeping it at a dull-red heat until every trace of gas-bells has disappeared. The fused salt is poured into a platinum basin, allowed to cool, broken up, and used in this condition. The neutrality of the fused salt was made sure of by dissolving 2 grms. in water, and titrating the "alkali" with very dilute standard solutions of hydrochloric acid and caustic potash, in the presence of aurine as an indicator. The "alkali" was found equivalent to $\frac{1}{220} \times \frac{1}{2} \mathrm{~K}_{2} \mathrm{O}$ mgs. $=0.2 \mathrm{mg}$, or rather $n i l$.

Pure Sal-Ammoniac.

See the section on chloroplatinate of ammonium, page 627 .

\section{Rubidium Chloride.}

See the section on its chloroplatinate, page 618 .

Standard Solution of Nitrate of Silver.

As a rule, we prepared this reagent from pure nitrate, in which the water had been determined immediately before use. A quantity, containing $n \times 17$ grms. of dry nitrate, was dissolved to $n$ litres. For the standardisation of the solution a standard solution of chloride of potassium was made synthetically by dissolving I $^{\frac{1}{0}} \mathrm{KCl}$ grms. in water, diluting to 1 litre, and weighing the 
solution produced before the last mixing. 50 (or 100) c.c. of this solution were weighed into a tared phial, 51 (or 101) c.c. of silver solution added, the mixture reweighed to obtain the weight of the silver solution added, then shaken, and the chloride of silver allowed to settle in the dark. The supernatant liquor was decanted off, iron-alum added, and the exact quantity of dissolved silver determined by to-and-fro titration with decimal (i.e. centi-normal) solutions of sulphocyanate of ammonium and nitrate of silver. These solutions were standardised only by volume, and measured off in burettes. From the result and the specific gravity of the stronger silver solution, it was easy to calculate the exact number of grammes of the latter which precipitate $\frac{1}{10} \mathrm{KCl}=74.59$ grms. of chloride of potassium. This number was recorded as the "weightequivalent" of the reagent, and utilised in the calculation. In a perfectly similar manner we proceeded in our determination of chlorine by titration. The given solution of chloride was diluted to a known weight. A small fraction, weighed out, served for a preliminary titration of the chlorine in which the reagents* were merely measured by volume. In a larger aliquot part then the exact weight of the chlorine was determined by a method of gravimetric titration closely analogous to the one explained for the standardisation of the silver solution. This particular modification of VoLHARD's method having been worked out by one of us (long ago) for the determination of the chlorine in sea-water (see "Challenger" Memoirs, Physics and Chemistry, vol. i. in DitTmar's Report on the Composition of Ocean Water), it will be referred to as the Challenger method.

In most of our analyses we determined the chlorine by means of this titrimetric method; sometimes we checked the results by gravimetric determinations in other aliquot parts of the respective solutions. In a few cases, when we had only little material at our disposal, we combined VoLHARD's method with the ordinary gravimetric method. The chlorine was precipitated by means of a known sufficient weight of the standard silver solution, and the chloride of silver collected and weighed. In the filtrate and wash-waters the still unprecipitated silver was then determined by VoLHARD's method, with volumetrically adjusted decimal (centi-normal) solutions. As each such duplicate analysis was preceded by a preliminary titration of the chlorine, we had no difficulty in so adjusting the weight of silver solution to be added, that the excess of silver left unprecipitated could be conveniently determined in the way explained.

After this introduction, we will proceed next to report on a series of experiments for determining the composition of chloroplatinate of potassium as produced under conditions similar to those prevailing in certain forms of the corresponding analytical method.

* The silver solution and a volumetric deci-normal solution of sulphocyanate. 
CRITICAL EXPERIMENTS FOR DETERMINATION OF POTASSIUM, ETC. 567

\section{Experimentis on the Composition of Chloroplatinate of Potassium.}

(First Series.)

The general modus operandi was as follows :-A known weight of chloride of potassium (weighed out in the form of a gravimetrically standarised solution) was mixed with, in general, a slight or moderate excess of standard chloroplatinic acid solution, whose weight was determined and recorded likewise. After production of the chloroplatinate (in some way or other), the motherliquor was decanted and filtered off, the chloroplatinate washed (as a rule first with small instalments of water, then with alcohol), dried at a certain temperature, and weighed. The mother-liquor, after removal of the alcohol from the alcoholic portion, was diluted with water, placed in an Erlenmeyer flask, and the platinum reduced out by purified hydrogen in the wet way at 80 to $90^{\circ} \mathrm{C}$. After complete reduction, the hydrogen was displaced by carbonic acid (to avoid explosions), the platinum filtered off, ignited, and weighed. The filtrate and wash-waters were diluted to a certain weight, and aliquot weighed parts used for the repeated determination of the fixed chlorine, i.e., the chlorine present as $\mathrm{KCl}$. For this purpose the respective liquid was evaporated to dryness, the residue dried further at $130^{\circ}$, then moistened with water, the solution re-evaporated, and the residue heated again to $130^{\circ}$, so that the free hydrochloric acid could be assumed to be away, which point, however, was always made sure of by the application of a thread of delicate litmus-paper to the last solution. In the chloride of potassium thus isolated, the chlorine was determined ; generally by means of the "Challenger" method. In the later experiments a very small quantity of fixed chlorine present in the chloroplatinic acid used was determined in a large quantity of the respective reagent, and allowed for. By deducting the weight of platinum and chloride of potassium found in the mother-liquor from the weights originally taken, we obtained the weights that had passed into the precipitate.

In addition thereto, the precipitated chloroplatinate, as a rule at least, was analysed, more or less completely. Our method of analysis, in the earlier experiments, was to reduce the chloroplatinate in a boat standing within a combustion-tube, in hydrogen gas. To avoid loss the out-going gas was made to bubble through a little water contained in a bulbed U-tube. The contents of the U-tube were evaporated to dryness, the residue united with the aqueous extract of the ignited mass and the washings of the combustion-tube, the whole evaporated to dryness, the residue re-dissolved in water, and the small quantity of platinum, which invariably separated out (even from perfectly clear liquors) collected and weighed. In one case the chloride of potassium thus obtained was weighed directly; as a rule, we relied for it on the 
synthetical data. The platinum was washed, ignited in a small porcelain crucible, and weighed.

We soon, however, came to discard this method, and to reduce our chloroplatinates in the wet way. The chloroplatinate, after having been dried at a certain temperature and weighed, was placed in an Erlenmeyer flask, along with a sufficient quantity of water to dissolve the chloroplatinate in the heat, the platinum reduced out in the wet way, filtered off, and weighed,* and aliquot parts of the filtrate used for the determination of (in general) the fixed and the total chlorine.

In the following reports on the several experiments, the symbol A stands for the weight of chloride of potassium used in the synthesis; $P$ for the approximate weight of platinum used, as chloroplatinic acid, per $2 \mathrm{KCl}=149 \cdot 18$ parts of chloride of potassium; $M$ for the exact weight of chloroplatinate which, according to analysis or synthesis, contains $2 \mathrm{KCl}$ parts of chloride of potassium.

\section{Experiment I. (St Petersburg Metal).}

$\mathrm{A}=0.74 \mathrm{grm}$. (about) ; $\mathrm{P}=201 \cdot 6$. Platinum solution poured into that of the chloride of potassium. Total volume of mixture, 40 c.c. The whole was evaporated to 2-3 c.c., and, as no excess of platinum was visible, another 1 c.c. of platinum solution was added (and its water evaporated away); hence, finally, $\mathrm{P}=209 \cdot 3$. The residue was washed with absolute alcohol (which became distinctly yellow); the chloroplatinate dried at $110^{\circ}$, weighed, and analysed in the dry way.

The results, referred to $2 \mathrm{KCl}$ parts, were as follows (in the analysis of the precipitate only the platinum was determined) :-

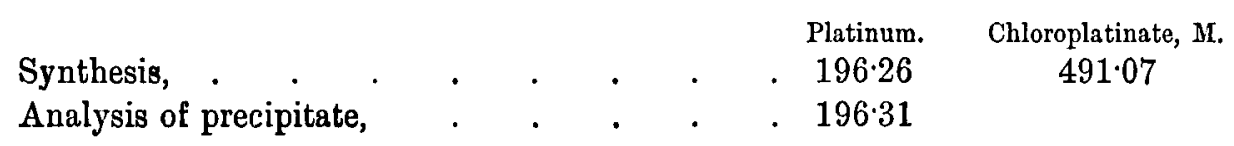

Hence $\mathrm{Pt}=196 \cdot 29$; and thence, by calculation, $\mathrm{PtCl}_{6} \mathrm{~K}_{2}=487 \cdot 29=\mathrm{M}-3 \cdot 78$; hence water, \&c., in the chloroplatinate $=0.700$ per cent.

\section{Experiment II. (Matthey's Metal).}

$\mathrm{C}=1.85495 ; \mathrm{P}=218.4$. Platinum poured into potassium salt. Evaporation to about 5 c.c., then addition of absolute alcohol, as in Experiment $I$. Precipitate washed with absolute alcohol, and dried, first at $110^{\circ}$, then at $130^{\circ}$. Reduction by hydrogen effected in the dry way. Combined chloride of

* Sometimes a film of platinum adheres firmly to the sides of the flask. This is easily recovered by dissolving it in aqua regia, evaporating to dryness in a porcelain crucible, and igniting the residue. 
CRITICAL EXPERIMENTS FOR DETERMINATION OF POTASSIUM, ETC. 569

potassium determined only synthetically; the platinum both ways. Results, referred to $2 \mathrm{KCl}$ parts, were-

\begin{tabular}{|c|c|c|}
\hline & Platinum. & $\begin{array}{l}\text { Chloroplatinate, dried at } \\
110^{\circ} \text {. }\end{array}$ \\
\hline & $195 \cdot 71$ & $488 \cdot 43\left(=M^{\prime}\right)$ \\
\hline An & $195 \cdot 63$ & \\
\hline
\end{tabular}

Hence, by calculation, $\mathrm{P}=195 \cdot 67$, and $\mathrm{PtCl}_{6} \mathrm{~K}_{2}=486 \cdot 67$. Hence water, \&c. in $\mathrm{M}^{\prime},=1.76$ parts, or 0.360 per cent.

in $\mathrm{M}^{\prime \prime},=1 \cdot 61 \quad, \quad 0.330 \quad$,

Experiment III. (Matthey's Metal).

$A=1.85556 ; \mathrm{P}=206.4$ parts. The two reagent solutions were evaporated separately to about 30 c.c. each, and then mixed; the platinum being poured into the potassium salt. Mixture evaporated to 5 c.c., and allowed to stand over night. The precipitate washed by decanting filtration with three instalments of water, each $=3$ c.c.; 15 c.c. of absolute alcohol were then added, and the precipitate washed with absolute alcohol. The precipitate was dried at $130^{\circ}$, first for 5 hours, and then for other 24 hours; and in part of the final residue the water was determined divectly (see page 578). The combined chloride of potassium was determined only by synthesis; the platinum both ways. Found per $2 \mathrm{KCl}$ parts,

$$
\left.\begin{array}{lll}
\text { Platinum, by synthesis, } & =195.75 \\
\text { Platinum, by analysis, } & = & 195.56
\end{array}\right\} 195.66 .
$$

and for the weight of the chloroplatinate:-

$$
\begin{array}{ccc}
\multicolumn{2}{c}{\text { Dried at } 130^{\circ} \text { for }} & \text { An } \\
5 \text { hours. } & \text { Anhydrous, by water } \\
\mathrm{M}^{\prime}=487 \cdot 78 & \mathrm{M}^{\prime \prime}=487 \cdot 29 & \mathrm{M}^{\prime \prime \prime}=486.63
\end{array}
$$

From $\mathrm{Pt}=195 \cdot 66$; by calculation, $\mathrm{PtCl}_{6} \mathrm{~K}_{2}=486 \cdot 66$; hence water, \&c.,

$\begin{array}{lll}1.12 & 0.63 & -0.03 \\ \text { or } 0.230 & 0.129 & \text { nil per cent. }\end{array}$

Experiment IV. (Matthey's Metal).

This experiment was wrought side by side of Experiment III., on the same scale, and in the same way, except that the chloride of potassium was poured into the platinum, and that the excess used of the latter was a little greater. The two chloroplatinates were dried, side by side, in the same chamber, only while, after 29 hours at $130^{\circ}$, No. III. was taken out to be analysed, No. IV. was dried for an additional 12 hours at $150^{\circ}$. The water in the finally dried substance was determined dirertly (see page 578). During the heating process involved in the determination of the water, the salt decrepitated so much, that a reduction of the residue in hydrogen could not have been effected with

VOL. XXXIII. PART II. 
quantitative precision. Hence we relied entirely on the synthesis for both the fixed chlorine and the platinum.

Platinum taken, per $2 \mathrm{KCl}$ parts of chloride of potassium, $=214.3$ parts.

Found per $2 \mathrm{KCl}$ parts.

Platinum $=19579$, and chloroplatinate

\begin{tabular}{ccccc}
\multicolumn{5}{c}{ Dried at $130^{\circ}$ for } \\
M & 5 hours. & 29 hours. & hours, and at $150^{\circ}$ & Anhydrous Salt. \\
& 489.67 & 488.67 & 488.07 & 487.05
\end{tabular}

Now, for $\mathrm{Pt}=195 \cdot 79: \mathrm{PtCl}_{6} \mathrm{~K}_{2}=486 \cdot 79$.

Hence for water, \&c.,

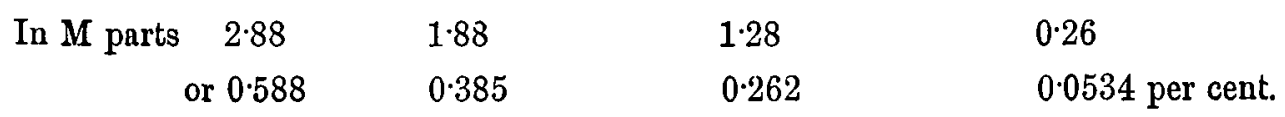

Experiment V. (Matthey's Metal).

$\mathrm{A}=3 \cdot 7822 ; \mathrm{P}=195 \cdot 0$.

The chloride of potassium was dissolved in 20 c.c. of water, and the platinum solution ( $107 \cdot 6$ c.c.) added, cold. Mixture allowed to stand over night, liquor decanted off through a small filter, precipitate washed with small successive instalments of water, until the small excess of either reagent, which could be presumed to be present, was sure to be washed away (washwater used, $=4 \times 5=20$ c.c.). The small portion of the precipitate which had gone on the filter was then washed with a little absolute alcohol, but these washings were collected by themselves. The aqueous filtrate and washings (some 130 c.c.) were evaporated to dryness over a water-bath in a platinum basin; the residue, along with the small quantity of chloroplatinate from the filter dissolved in boiling water (about 150 c.c.), and the solution cooled down finally in ice, to cause as much as possible of the chloroplatinate to crystallise out. The crystals "I." obtained were allowed to settle, the liquor decanted off through the original filter, and the filtrate evaporated down to 20 c.c. The solution was cooled down, and allowed to stand over night, when another smaller crop of crystals "II." came out. The mother-liquor was decanted off through the filter, and the three portions of recovered chloroplatinate washed systematically with small instalments of cold water, each equal to about 2 c.c. Each 2 c.c. of water went first on crystals "I.", then on crystals "II.", and lastly on and through the filter. Four such washings were carried out. The last mother-liquor and these washings were analysed as usual to find the weights of chloride of potassium and platinum to be deducted from the quantities originally taken, in order to find how much of each was contained in the three precipitates of chloroplatinate, namely, the bulk of the original precipitate (it, after drying at $130^{\circ}$, weighed 9.6244 grms.), and the two crops of chloroplatinate recovered from filter and mother-liquor. The weights of 
these latter were not determined, but from the total combined platinum in the three portions we can calculate the total weight of chloroplatinate synthetically analysed. The total platinum obtained amounted to 4.88266 grms., whence, if we go by Experiment "III.", and the " $M$ " for 5 hours' drying at $130^{\circ}$ given there as corresponding to $195 \cdot 66$ of platinum, we have $12 \cdot 1724 \mathrm{grms}$. for the total chloroplatinate, whence, by difference, 2.5480 grms. for the two minor portions conjointly.

Found, synthetically, per $2 \mathrm{KCl}$ parts, platinum $=196 \cdot 64$. Exact data for "M" absent.

Note.-The aqueous filtrate and washings contained $60.4 \mathrm{mgs}$. of platinum, which need 46.2 mgs. of chloride of potassium to be made into chloroplatinate ; instead of these $46 \cdot 2$, we found $77 \cdot 9 \mathrm{mgs}$.

Experiment Va. (Matthey's Metal).

$A=4.5385$ grms. weighed directly, and dissolved in 100 c.c. of water. $\mathbf{P}=203.4$ parts, calculated from the volume of platinum solution used, which was 125 c.c.

The chloride of potassiurn was poured into the platinum solution, and the mixture allowed to stand over night. Next morning the mother-liquor was decanted off through a small filter, and the precipitate washed three times, each time with 10 c.c. of water; 20 c.c. of absolute alcohol were then poured on the precipitate in the basin, the small portion from the filter washed in with absolute alcohol, and the whole allowed to stand for $\frac{1}{4}$ hour, to effect at least a partial dehydration. The precipitate was then washed with absolute alcohol, and next kept over oil of vitriol for half a day. It was then dried at $100^{\circ}$ for $\dot{a}$ short time, transferred to a flat platinum crucible, and dried for 4 hours at $110^{\circ}$, when its weight was found to be $12 \cdot 1063$ grms. This precipitate was put into a Geissler tube. A portion (A), amounting to 2.9398 grms., was however weighed out at once for analysis, and another (B) (2.9255 grms.), at the same time, for the determination of the water volatile at $150^{\circ}$, which, as we may state at once, amounted to 11.5 mgs., or 0.393 per cent. (after 23 hours' drying). The reduction of " $A$ " was conducted in the wet way with 200 c.c. of water, the platinum filtered off, and weighed; (it weighed 1.1768 grms.). Aliquot portions of the filtrate served for the determination of the fixed and total chlorine. The former was determined once; (found 0.42449 grms. per total solution); the latter twice; found 1.27143 , and 1.27122 , mean $=1.27133 \mathrm{grms}$. adopted. Hence we had, by calculation, per $2 \mathrm{KCl}$ parts-

$\begin{array}{cccc}\text { Platinum, } & \text { Loose Chlorine. } & \mathbf{M}_{110 \cdot} & \mathbf{M}_{150^{*}} \\ 196.58 & 1.99496 \times \mathrm{Cl}_{2} & 491 \cdot 087 & 489 \cdot 166\end{array}$

" $\mathrm{M}_{110}$ " stands for chloroplatinate dried for 4 hours at $110^{\circ} \mathrm{C}$.; 
" $\mathrm{M}_{150}$ " for the same, dried in addition for 23 hours at $150^{\circ} \mathrm{C}$.

The number for the loose chlorine suggests the presence of $0.00504 \times(\mathrm{O}=16$ parts of oxygen) in the $\mathrm{PtX}_{4}$ part of the salt. Admitting this, we have, if $\mathrm{Pt}=196.58$, for the composition of $\mathrm{M}$ parts of this kind of chloroplatinate-

\begin{tabular}{lllllllr} 
Platinum, &. &. &. &. &. &. & 196.58 \\
$2 \mathrm{KCl},$. &. &. &. &. &. &. & $149 \cdot 18$ \\
$1.99496 \times \mathrm{Cl}_{2}$, &. &. &. &. &. &. & 141.46 \\
$0.00504 \times 0$, &. &. &. &. &. &. & 0.08 \\
& & & & & & & \\
\hline
\end{tabular}

Hence water, \&c. in.

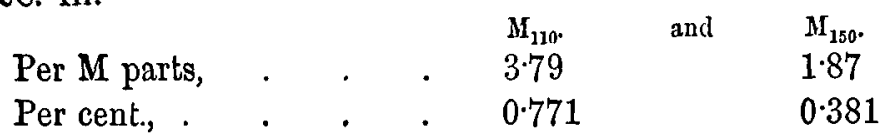

The oxychloride oxygen amounts to very little, supposing it to be present at all. Taking the two determinations of the total chlorine as so many determinations of three times the fixed chlorine, and combining them with the direct determination of the latter, we had :-

Mean value of fixed chlorine $=0.42401$; and for $2 \mathrm{KCl}$ parts

$\begin{array}{lllllllll}\text { Platinum, } & . & . & . & . & . & . & . & 196 \cdot 80 \\ 2 \mathrm{KCl}, . & . & . & . & . & . & . & . & 149 \cdot 18 \\ 4 \mathrm{Cl}, . & . & . & . & . & . & . & . & 141 \cdot 82 \\ \mathrm{PtCl}_{8} \mathrm{~K}_{2}, & . & . & . & . & . & . & . & 487 \cdot 80\end{array}$

and for the values $M$,

$$
M_{110}=491 \cdot 64 ; M_{150}=489 \cdot 72 .
$$

and for water, etc., in these,

$\begin{array}{lllllll}\text { Per M parts, } & \text {. } & \text {. } & \text {. } & \text {. } & 3.84 & 1.92\end{array}$

The results remain substantially the same.

Experiment VI. (Matthey's Metal).

At the time when this experiment was planned, we had arrived at the conviction that the atomic weight of platinum is very nearly, if not exactly, equal to 196, and the object of the experiment was to test this number in the most direct manner possible, namely, as follows :-

$0.7548 \mathrm{grm}$. of chloride of potassium was dissolved in 20 c.c. of water, and the solution transferred to a tared glass-stoppered bottle of about 120 c.c. capacity. 20.5 c.c. of standard platinum solution were then measured out, weighed exactly, and added to the chloride of potassium. From the weight and the known titre of the solution, it followed that the weight of platinum added amounted to $0.99086 \mathrm{grm}$. or to 195.9 per $2 \mathrm{KCl}$. The idea was to let the chloroplatinate separate out as far as possible, to draw off some 
of the clear liquor, and, by its analysis for platinum and fixed chlorine, prove the absence of excess, or determine such small excess as there might be, of either reagent. But very little chloroplatinate did settle out. We therefore added enough of absolute alcohol to nearly fill the bottle, shook up the contents, and allowed to stand, stopper on, over night. Next morning the whole was weighed, as much as possible of the clear liquor drawn off, weighed, (in a stoppered phial), and analysed for platinum, total chlorine, and fixed chlorine. For this purpose, the solution (after addition of water but without removing its alcohol), was kept at a temperature near to, but below, its boiling-point, within a conical flask, through which a very slow current of hydrogen was constantly passing. The out-going hydrogen was made to bubble through a quantity of water contained in a bulbed U-tube, to catch any hydrochloric acid that might go off. Let us at once state that no chlorine was found in this wash-water after the experiment. The platinum was filtered off and weighed; the filtrate diluted to a known weight, and aliquot parts used for the determination of the fixed and total chlorine. As there was not enough of material for repeating either determination, the chlorine, in each case, was precipitated with a known weight of standard nitrate of silver, the precipitated chloride of silver weighed, and the excess of silver in the filtrate determined by Volhard's method, to check the gravimetric determination. From the weight of platinum obtained, the corresponding weight of chloroplatinate of potassium was calculated, the resulting number multiplied by the ratio of bottle contents to liquor analysed, and the result deducted from the total chloroplatinate (solid or dissolved) calculated from the total platinum used, to obtain a first approximation to the weight of solid chloroplatinate in the precipitation bottle, at the time when the liquor was withdrawn. The result, when subtracted from the weight of the total contents, gave an approximation to the weight of the total mother-liquor. From this weight a second approximation to the dissolved chloroplatinate was obtained, and thus a second approximation to the weight of the solid chloroplatinate, and consequently also to that of the mother-liquor. This second result served for the reduction of the chlorines formed in the drawn off part to the total liquor.

We might have stated before that the platinum solution, before being used, was analysed to find the quantities of platinum, total chlorine, and fixed chlorine present in the weight of reagent employed. To enable the reader to form his own opinion on the probable uncertainty in the final results, we quote the following numbers:-

Found, for the total liquor drawn off-

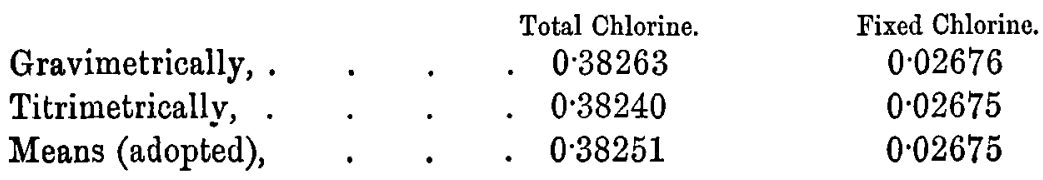


Calculated weight of total mother-liquor $=114.272$ grms.;

Weight of drawn-off liquor analysed, $\quad=99718$. Hence,

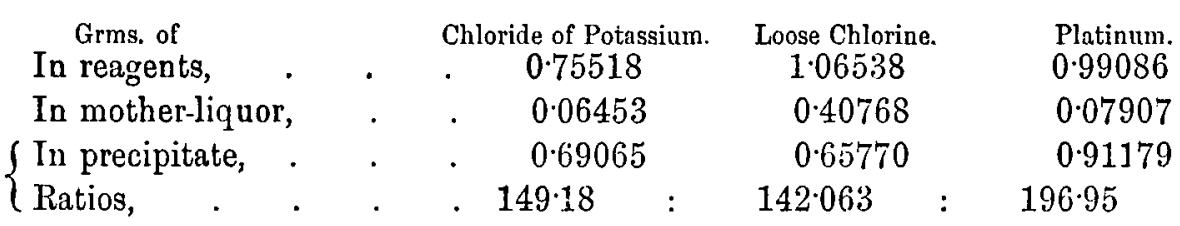

The loose chlorine equals $4.00685 \times \mathrm{Cl}$, which, considering the complexity of the method, may be accepted as a sufficient approximation to 4.0000 .

The value $\mathrm{Pt}$, calculated from the loose chlorine, comes to 196.61; calculated from the total chlorine, to $\mathbf{1 9 6} \cdot 72$. This last number probably is the most exact of the three; but any are considerably above 196 .

The following experiment was made with the view of finding a lower limit for the value of the constant:-

\section{Experiment VII.}

9.3125 grms. of chloride of potassium were dissolved in 50 c.c. of water, and mixed with a measured volume of standard platinum solution, containing $2 \cdot 45$ grms. of metal, i.e., only about one-fifth of what the chloride of potassium would demand for its conversion into chloroplatinate. Total volume of mixture $=100$ c.c. about. After a night's standing a large precipitate of chloroplatinate had settled out; it was washed with small successive instalments of water, until the excess of chloride of potassium, by calculation, was reduced to about $10 \mathrm{mgs}$. The washing was then continued with a cold-saturated solution of chloroplatinate of potassium. About 60 c.c. of this solution were used in all; after it followed two washings with 50 per cent., and at last a few with absolute, alcohol. The precipitate was dried, first for a night over vitriol, and then at $100^{\circ}$ until constant within 4 to $5 \mathrm{mgs}$. It was then divided into two approximately equal parts, and both analysed by reduction with hydrogen in the wet way. That combination of the gravimetric with the titrimetric modus, which had served in Experiment VI., was used again.

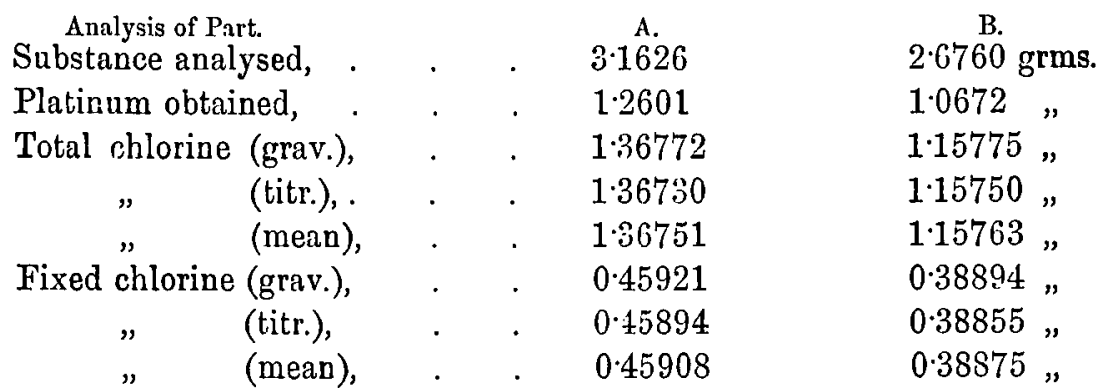

Referring in both cases to $2 \mathrm{KCl}$ parts, we have-

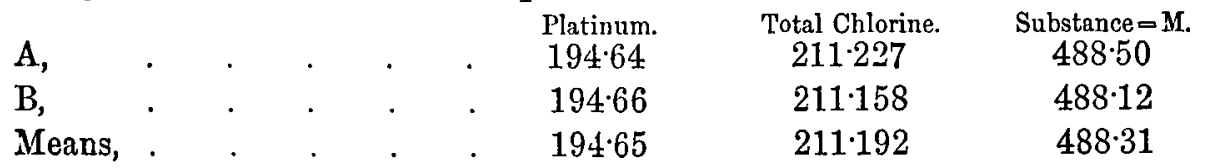


CRITICAL EXPERIMENTS FOR DETERMINATION OF POTASSIUM, ETC. $\mathbf{5 7 5}$

Adopting the mean, it would follow that $\mathrm{Pt}=194.65$, and that this $\mathrm{Pt}$ is combined with $211.192=5.95663 \times \mathrm{Cl}$, supplemented by $0.04337 \times\left(\mathrm{OH}\right.$ or $\left.\frac{1}{2} \mathrm{O}\right)$. But we have no excuse for presuming that oxychloride-oxygen is present; on the other hand, we have good reason for suspecting the presence of surplus chloride of potassium. Referring the mean results to $4 \times \mathrm{Cl}$ parts of loose chlorine, we have

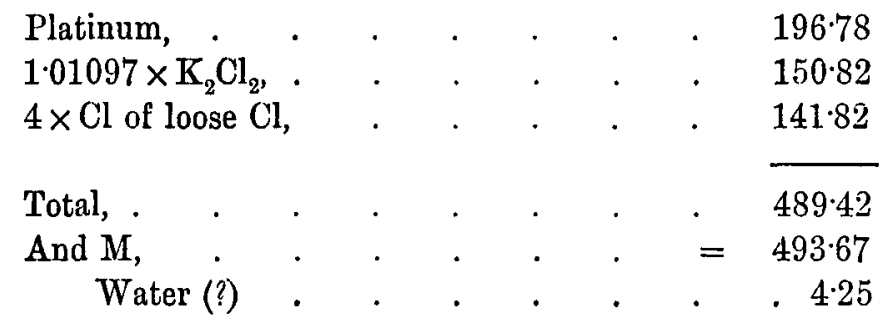

This, we believe, is the correct mode of interpreting the analysis.

It does not follow that $\mathbf{1 9 6 . 7 8}$ is the true atomic weight of platinum.

\section{Experiment VIII. (Matthey's Metal).}

All the experiments reported on so far were made with platinum solutions, made by means of the ordinary aqua regia process. We have reason to suspect that these solutions contained small quantities of the nitroso-compound $\mathrm{PtCl}_{6}(\mathrm{NO})_{2}$, which may have had an evil. effect, although it is not quite easy to see how this can have been the case. However this may be, the following experiments (VIII. and IX.) are free of this flaw, because they were carried out with platinum solution made by the chlorine process (see page 565). Their most important feature, however, is that a very considerable excess of chloroplatinic acid was used in the preparation of the chloroplatinates.

In Experiment VIII. 3.045 grms. of chloride of potassium were dissolved in 40 c.c. of water, and precipitated with 120 c.c. of a platinum solution containing $6^{\circ} 0$ grms. of platinum, so that the platinum added per $2 \mathrm{KCl}$ parts amounted to 294.0 parts. The potassium salt was poured into the platinum solution, the mixture allowed to stand over night, the precipitate washed by decanting filtration, first with 5 c.c. of water (i.e., chloroplatinic acid solution), and then with absolute alcohol; the aqueous and alcoholic washings were preserved separately. The precipitate was dried at $100^{\circ}$ to $105^{\circ}$ in a watchglass for $4 \frac{1}{2}$ hours, and then for another hour in a Geissler tube at the same temperature. The precipitate acquired a constant weight in a remarkably short time. After having been dried, it was divided into three parts, $\mathrm{A}, \mathrm{B}, \mathrm{C}$.

$\mathbf{A}=3.3511$ grms. was devoted to a direct determination of the water. Water obtained $=30.8$ mgs. $=0.919$ per cent. 
B ( $=2 \cdot 8375)$ and C (2.8884 grms.) were dissolved separately in hot water and reduced by hydrogen, for the determination of the platinum, the fixed chlorine, and the total chlorine.

The results were as follows :-

Analysis of " $B$."-Substance $=2 \cdot 8375$; platinum therefrom $=1 \cdot 1312$. Total chlorine [in fraction analysed, by titration $=0.308683$; gravimetrically $=0.308791$ : mean $=0.308737$; whence, for the whole], 1.22855 grms. Fixed chlorine [by titration $=0.306219$; gravimetrically $=0.306615$; whence, for the whole], 0.409294 grms.

Hence per $2 \mathrm{KCl}$ parts :-

\begin{tabular}{|c|c|c|}
\hline Platinum. & Total Chlorine. & $\begin{array}{l}\text { Substance analysed. } \\
\text { Dried at } 100^{\circ} \mathrm{C} \text { Anhydrous. }\end{array}$ \\
\hline $195 \cdot 98$ & $212 \cdot 85=6.0033 \mathrm{Cl}$. & $491.60 \quad 487.08$ \\
\hline
\end{tabular}

Sum of components $=486.98$, which leaves no room for oxychloride oxygen.

Analysis of " $C$."-Substance $=2.8884$; platinum $=1 \cdot 1518 . \quad$ Total chlorine [calculated from mean of 0.314118 and 0.314255 ], 1.25006. Fixed chlorine [calculated from mean of 0.312018 and 0.312574 ], 0.417213 .

Hence per $2 \mathrm{KCl}$ parts :-

\begin{tabular}{cccc} 
Platinum. & Total Chlorine. & \multicolumn{2}{c}{ Substance. } \\
195.76 & $212 \cdot 46=5.9924 \times \mathrm{Cl}$. & Dried at $100^{\circ} \mathrm{C}$. & Anhydrous. \\
& 490.92 & 486.40
\end{tabular}

Sum of components $=486.76$, or adding in $\left(0.0076 \times \frac{1}{2} 0=0.061\right) 486.82$. But the small chlorine-deficit had better be viewed as an observational error, like the excess in the analysis of "B." The filtrate from the chloroplatinate $(A+B+C)$, which still contained some 2 grms. of platinum, was utilised for

\section{Experiment IX.}

thus. It was mixed with $1 \mathrm{grm}$. of chloride of potassium, i.e., two-thirds of what the 2 grms. of platinum required for their conversion into chloroplatinate and the mixture wrought in pretty much the way prescribed by $\mathrm{Mr}$ TATLOCK in his form of the chloroplatinate process for determining potassium; i.e., it was evaporated to near dryness on a water-bath, some added water evaporated over the residue to eliminate the free hydrochloric acid as fully as possible, and the residue, after cooling, digested in the cold with 10 c.c. of water, i.e., virtually 10 c.c. of a 6 to 7 per cent.* platinum solution, for an hour. The liquor was then decanted off through a filter, and the precipitate washed, first twice with small added volumes of 5 per cent.* platinum solution, and at last with 95 per cent. (by weight) alcohol. It was dried at $100^{\circ}$ in a Geissler tube (the weight became constant very soon) and weighed. It weighed 4.0487 grms. It was divided into three parts, A, B, and C.

* Meaning a solution containing so many centigrammes of metallic platinum per c.c. 
"A" was used for a direct determination of the water. 1.3573 grms. of substance gave $1.1 \mathrm{mgs}$, or 0.081 per cent. " $\mathrm{B}$ " and " $\mathrm{C}$ " were analysed by wet-way reduction. Found for

$\begin{array}{lcccc} & & \text { "B." } & \text { "C." } & \text { Mean. } \\ \text { Substance, . . . } & . & 1 \cdot 0990 & 1.5917 & \\ \text { Platinum obtained, . } & . & 0.4416 & 0.6403 & \\ \text { Substance per 1 grm. of platinum, } & 2 \cdot 4887 & 2 \cdot 4859 & 244873\end{array}$

The agreement being satisfactory, the filtrates from the platinum were mixed, and used for the determination of the fixed, and of the total, chlorine.

Results. -Substance $=2.6912$ (by direct weighing preceding the dividing into "B" and "C"). Total chlorine [calculated from the mean of 0.23554 and $0 \cdot 23582$ ] $=1 \cdot 17466$. Fixed chlorine [calculated from the mean of 0.23462 and $0.23501]=0.39223$.

Hence per $2 \mathrm{KCl}$ parts :-

\begin{tabular}{|c|c|c|}
\hline & & Substance. \\
\hline Platiu & Total Chlorine. & $\begin{array}{c}\text { Dried at } 100^{\circ} \text {. Anhydrous } \\
\mathbf{M}^{\prime} .\end{array}$ \\
\hline $95 \cdot 59$ & $212.362=5.9896 \times \mathrm{Cl}$, & $486 \cdot 53$ \\
\hline
\end{tabular}

Neglecting the small chlorine-deficit, we have for the sum of components 486.59, which, singularly, agrees better with $\mathrm{M}^{\prime}$ than with $\mathrm{M}^{\prime \prime}$. Perhaps the $1.1 \mathrm{mgs}$. of water found in " $A$ " was the result of observational errors,-water absorbed after weighing of the substance, or water out of the joints, \&c.

In the preparation of the two chloroplatinates VIII. and IX. we were very much struck by the promptitude with which they acquired a constant weight in the drying chamber; all our previous chloroplatinates used to continue losing weight for hours and hours, and hardly ever really exhibited absolute constancy of weight.

The most remarkable feature in these chloroplatinates, however, is, that although produced in the midst of a very large excess of chloroplatinic acid, they contained rather less platinum per $2 \mathrm{KCl}$ parts than we had found in the chloroplatinates previously produced in the presence of small excesses, or even negative excesses, of the platinic reagent. Yet it does not follow that even those quasi-exceptional chloroplatinates (of Experiments VIII. and IX.) were free of surplus platinum. At the time when Experiments VIII. and IX. were planned, this question had already been expiscated experimentally to some extent by special experiments on chloroplatinates V. and Va., which were done at the time when the analysis of $\mathrm{V}$. and $\mathrm{V} a$. were carried out, but which we prefer, in this memoir, to treat of separately in the following section.

VOL. XXXIII. PART II. 


\section{On the Composition of Chloroplatinate of Potassium.}

\section{Second Series of Experiments.}

Chloroplatinate of potassium, as has been long known, is liable to contain water, and indeed in most cases does contain water, so intimately combined with the rest that it cannot be completely expelled at even $150^{\circ}$. That this water should all be present as such, as a mere enclosure within the crystals, is difficult to believe; it is more likely to be present, at least partly, in the form of hydroxyl, functioning as part of the loose chlorine in the ideal substance. From certain observations of SEUBERT's, indeed, it appears that whenever chloroplatinate of potassium is recrystallised from hot water, part of the chlorine passes into solution, and is replaced, of course, by its equivalent of hyclroxyl or oxygen. That this exchange should take place only in hot, and not at all in cold, solutions, is by no means probable. Any chloroplatinate is liable to be thus contaminated, and as long as its purity is not proved, and quite apart from any free chloride of potassium, or surplus platinum in this form or that, which may adhere to it, must be looked upon as a (mixed) oxychloride of the general formula $\mathrm{PtCl}_{(6-2 y)} \mathrm{O}_{y} \cdot \mathrm{K}_{2}+x \mathrm{H}_{2} \mathrm{O}$, where $y$ of course is a fractional number, and $x$ may be greater than $y$, because the salt may contain combined water in addition to the water present as hydroxyl. To be able to make a direct and complete analysis of a "chloroplatinate," we must have methods for the direct determination of the water and of the oxychloride-oxygen.

The determination of the water presents no difficulty; it indeed is so easy that we wonder that SEUBERT did not effect it with his chloroplatinate of potassium, and thus remove the cloud of uncertainty which hangs over those of his calculations of the atomic weight of platinum which are based on the ratio of platinum to non-platinum in the chloroplatinate. All that is required is, from a known weight of substance, contained in a porcelain boat standing in a combustion-tube, to expel what goes off at a dull red heat, to remove the liberated chlorine by passing the volatile products through a spiral of red-hot sheet silver, placed in the exit-end of the combustion-tube, to collect the thus purified water in a tared chloride of calcium tube, and weigh it. The water determinations referred to in the above reports were carried out in this manner.* To avoid the uncertainties arising from the use of cork joints, the exit end of the combustion-tube was drawn out to quill size, and the entrance end of the chloride of calcium tube joined on by means of a piece of indiarubber, in such a manner that only a narrow line of the latter was exposed to the out-going vapours. This joint, during the whole of the operation, was

* The water determination in Experiment III. forms an exception, in this sense, that the substance was heated in a current of nitrogen, and the products filtered through a stopper of copper gauze (instead of metallic silver). 
kept at $100^{\circ}$ to $105^{\circ}$ by means of a small chamber made of asbestos pasteboard, which enclosed it ; and the first step in each analysis was to make this joint anhydrous by passing a current of chloride of calcium dry air through the (heated) tube, until the chloride of calcium tube attached to its end ceased to gain weight, taking care to have the silver spiral at a red heat during an earlier stage of this preliminary operation. The expulsion of the water of the substance was effected in a slow current of chloride of calcium dry air.

The direct determination of the oxychloride-oxygen is a far more difficult problem, which we did not succeed in solving. To keep other chemists from wasting their time, we will shortly sketch out the two methods which we tried.

First Method.-A known weight of the chloroplatinate (contained in a boat standing within a combustion-tube, which has a spiral of red-hot sheet silver near its exit end) is heated to redness in a current of carbonic oxide, with the view of determining the water as such, and any surplus oxygen as carbonic acid. The former is collected in chloride of calcium, the latter in an evacuated flask containing a measured volume of standard baryta-water to be determined titrimetrically in the way which one of us employed successfully in the analysis of sea-water (see "Challenger Memoir"; also Dittmar's Exercises in Quantitative Analysis, section on Sea-water Analysis, pages 227-230). To test the method, we applied it to a known weight of pure fused chloride of silver. The carbonic oxide was prepared from oxalic acid, and kept in a Pisani gas-holder over strongly alkaline pyrogallate of soda. Certain other precautions which were taken are too obvious to be stated; suffice it to say that we obtained $16 \mathrm{mgs}$. of carbonic acid from a substance containing no oxygen.*

Second Method.-A known weight of the substance is heated in a current of pure hydrogen to obtain all the oxygen (present as water, or in other forms) as water, which is collected in a U-tube charged with pumice and oil of vitriol. By avoiding all cork-joints we had no difficulty in collecting our water in that U-tube, without any hydrochloric acid condensing along with it. To obtain air-free hydrogen, we employed a Kipp apparatus, charged with zinc and boiled out dilute sulphuric acid, and so communicating with a supplementary "Kipp," discharging hydrogen, as to maintain an atmosphere of hydrogen in the upper bulb of the other. The gas obtained was passed through a series of U-tubes charged with sulphuric acid and pumice, and from the last U-tube direct into a combustion-tube containing red-hot copper-gauze (and drawn out at both ends to avoid cork joints), and thence again through a U-tube containing sulphuric acid and pumice ; from this last tube the gas entered the combustiontube to do duty. This method, like the first, was rehearsed with pure chloride of silver, and the result was that the chloride of silver yielded a very

* No doubt some $\mathrm{COCl}_{2}$ had been produced from the $\mathrm{CO}$ and $\mathrm{AgCl}$, and not all been decomposed by the silver spiral, although we operated at the highest temperature permissible in a Bohemian glass tube. 
appreciable proportion of oxygen! (2.03 grms. gave $15.6 \mathrm{mgs}$.). Where this oxygen came from we are unable to say; perhaps it came out of the glass of the combustion-tube. If SснӧNBEIN were still alive he would perhaps say that the oxygen came out of the chloride of silver; we need not add that this is not our explanation of the phenomenon.

While we are compiling this memoir, it strikes us that the oxygen in a chloroplatinate might perhaps be determined by heating it, behind red-hot silver, in a combustion-tube connected with, and previously evacuated by means of, a Sprengel pump. The chlorine should be retained as $\mathrm{AgCl}$, and the oxygen go off as such (with the water), so that it could be collected, determined, and identified by the methods of gas analysis. Unfortunately the idea did not present itself to our minds at the time, and we had to rely on an obvious indirect method for determining the oxygen of a chloroplatinate. This is what we did in the following experiments.

Supplement to Experiment V. of First Series.

The principal chloroplatinate produced in this experiment,* while being dried at $130^{\circ}$, became slightly discoloured from some unexplained cause. Hence, instead of analysing it according to our general plan, we wrought it as follows :-

The dried precipitate was dissolved in water in a large platinum basin, the solution filtered, and the filtrate allowed to cool with occasional agitation. The crystalline deposit was washed twice, each time with 10 c.c. of water. It was then recrystallised from hot water. The crystals obtained were dried at $120^{\circ} \mathrm{C}$., weighed, dissolved in water, and reduced in hydrogen in the wet-way, to determine the platinum and the two chlorines.

The results were as follows :-

\begin{tabular}{|c|c|c|c|c|}
\hline Absolute weights, & $\begin{array}{c}\text { Fixed Chlorine. } \\
0.52125\end{array}$ & $\begin{array}{r}\text { Loose Chlorine. } \\
1.02916\end{array}$ & $\begin{array}{l}\text { Platinum. } \\
1.4374\end{array}$ & $\begin{array}{c}\text { Substance. } \\
3 \cdot 5704 \text { grms }\end{array}$ \\
\hline Relative & - $\quad \mathrm{Cl}_{2}=70.91$ & 140.005 & $195 \cdot 54$ & $485 \cdot 71 \quad$ \\
\hline
\end{tabular}

The chlorine-deficit is too great to be explained by observational errors; the salt must be assumed to have contained 0.0512 equivalents of oxygen, instead of chlorine, per molecule.

Admitting this, we have, for the composition of $485 \cdot 71$ parts,

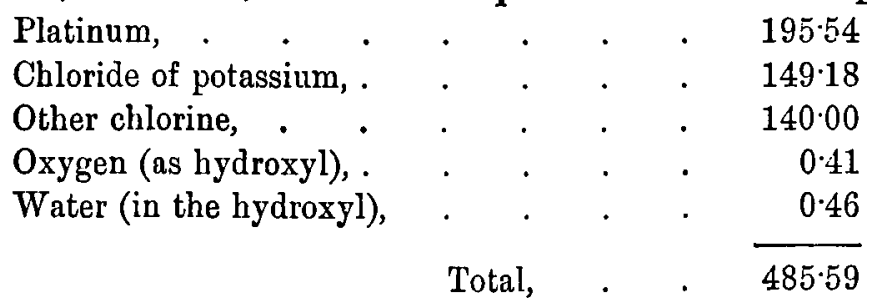

instead of $485 \cdot 71$, which is very satisfactory.

* We mean the bulk of the original precipitate; i.e., that part of it which did not pass on the filter, and which, as stated on page 570 , amounted to $9.6244 \mathrm{grms}$. after drying at $130^{\circ} \mathrm{C}$. 
The two mother-liquors derived from the recrystallisations were analysed (by wet-way reduction in hydrogen, \&c.), so as to determine the absolute quantities of platinum, fixed chlorine, and loose chlorine contained in them.

The first-mother liquor gave :-

\begin{tabular}{|c|c|c|c|c|}
\hline \multicolumn{2}{|c|}{ Absolute weights, } & $\begin{array}{l}\text { Platinum. } \\
0.7914\end{array}$ & $\begin{array}{l}\text { Fixed Chlorine. } \\
0.28214\end{array}$ & $\begin{array}{l}\text { Loose Chlorine. } \\
0.59644\end{array}$ \\
\hline Relative & $"$ & $196 \cdot 67$ & $1.9772 \times \mathrm{Cl}$ & $4 \cdot 1798 \times \mathrm{Cl}$ \\
\hline ” & $"$ & 194.00 & $1.951 \times \mathrm{Cl}$ & $4 \cdot 124 \times \mathrm{Cl}$ \\
\hline
\end{tabular}

The number 196.67 for $\mathrm{Pt}$ was chosen at the time, because it resulted from the synthesis of the original chloroplatinate * we now utilise it as an upper limit, while 194 is used as a lower limit, for the unknown true "Pt." Either mode of calculation leads to the conclusion that the liquor contained chloroplatinic acid and (really free) hydrochloric acid besides $\mathrm{PtCl}_{6} \mathrm{H}_{2}$.

These analyses consequently prove, what SEuberT only surmised, namely, that a chloroplatinate recrystallised from hot water contains oxygen in place of part of the chlorine of its $\mathrm{PtCl}_{4}$, and that the mother-liquor contains hydrochloric acid.

Before inquiring into the origin of the chloroplatinic acid, let us give the results of the analysis of the second mother-liquor. It contained-

$\begin{array}{cllll} & & \text { Platinum. } & \text { Fixed Chlorine. } & \text { Loose Chlorine. } \\ \text { Absolute weights, } & . & 0.5249 & 0.19199 & 0.36097 \\ \text { Relative " } & . & 196.67 & 2.0286 \times \mathrm{Cl} & 3.8140 \times \mathrm{Cl} \\ \quad " & . & 194.00 & 2.0014 & 3.029 \times \mathrm{Cl}\end{array}$

Either mode of calculation brings out oxychloride-oxygen, and with $\mathrm{Pt}=196.67$ we obtain, moreover (for so much platinum), $0.0286 \times \mathrm{KCl}$ of surplus chloride of potassium. But the silver solution used for the determination of the fixed chlorine (in the respective fraction of the filtrate from the platinum), amounted to only $27 \cdot 3$ grms. ; and either of the two numbers for the fixed chlorine (per Pt parts of platinum) must be considered uncertain by about \pm 0.002 of its value (because the weight of the platinum cannot be presumed to be free of error). Correcting it down by 0.002 of its amount, and reducing to $\mathrm{Pt}=195.5$ (which number we ultimately came to adopt as the most probable value, vide infra), we have-

$\begin{array}{ccc}\text { Platinum. } & \text { Fixed Chlorine. } & \text { Loose Chlorine. } \\ 195 \cdot 50 & 2 \cdot 0128 \times \mathrm{Cl} & 3 \cdot 7920 \times \mathrm{Cl}\end{array}$

It really would appear that a little chloride of potassium has been eliminated in the second recrystallisation; but it is not permissible to draw such a weighty conclusion from a single analysis made on such a small scale. We prefer to look upon the fraction 0.0128 as resulting from observational errors.

\footnotetext{
* And a small slip in a calculation; the correctly calculated value is 196.64 .
} 
Supplement to Experiment Va. of First Series.

Of the 12 grms. of chloroplatinate produced in this experiment, the greater part was used for the following experiments :-

6.2356 grms. of chloroplatinate, dried at $110^{\circ}$, was dissolved in about 250 c.c. of boiling water in a platinum basin, and the solution allowed to stand (for about $\mathbf{4 0}$ hours), when a large crop of crystals was found to have separated out. These were washed thrice with small instalments of water and put aside as "Crystals C." The mother-liquor was distilled down, in a flask provided with a ground-in alembic and attached to a Liebig's condenser, to about 25 c.c. The distillate contained a small quantity of hydrochloric acid, which was determined gravimetrically by nitrate of silver; its chlorine amounted to $0.87 \mathrm{mg}$.

The contents of the flask, on cooling, deposited a crop of crystals which were collected and put aside as "E," the mother-liquor being labelled "D." Each of the products was analysed in toto (by reduction by hydrogen in the wet-way) so as to obtain the absolute weights of platinum, \&c.

$$
\text { Crystals "C." }
$$

Substance unweighed. Platinum 1.9329. Total chlorine* 2.07648 and 2.07725; mean, 2.07686 . Fixed chlorine 0.46467 and 0.46450 ; mean 0.464585 per fraction analysed. For the whole 0.701157.

Hence per $2 \mathrm{KCl}$ parts :-

$$
\begin{array}{ccc}
\text { Platinum. } & \text { Loose Chlorine. } & \text { Oxygen (calculated). } \\
195.48 & 139 \cdot 129 & \\
& \text { or } 3.9241 \times \mathrm{Cl} & 0.0759 \times \frac{1}{2} \mathrm{O}
\end{array}
$$

About 2 per cent. of the loose chlorine is replaced by oxygen.

$$
\begin{aligned}
& \text { Crystals " } E . " \\
& \begin{array}{lllll} 
& \text { Fixed Chlorine. } & \text { Loose Chlorine. } & \text { Platinum. } & \text { Oxygen. } \\
\text { Absolute weights, } 0.15260 & 0.29754 & 0.4195 & \\
\text { Relative " } \quad 70.91 & 138.26 & 194.93 & \\
& & =3.8996 \times \mathrm{Cl} & & 0.1004 \times \frac{1}{2} \mathrm{O}
\end{array}
\end{aligned}
$$

$\begin{array}{lccc} & \text { Mother-liquor “ } D . " \\ & \text { Platinum. } & \text { Fixed Chlorine. } & \text { Loose Chlorine. } \\ \text { Absolute weights, } & .0 \cdot 1441 & 0 \cdot 05044 & 0 \cdot 11940 \\ \text { Relative " } & 195.00+ & 68 \cdot 253 & 161.58 \\ & & \text { or } 1.9250 \times \mathrm{Cl} & \text { or } 4.5574 \times \mathrm{Cl}\end{array}$

Hence, for the composition of the solution (if we take $\mathrm{Pt}=195$, as we

* The $\mathrm{AgCl}$ in the titrations had a tinge of pink indicating platinum.

$\dagger$ Including the $0.87 \mathrm{mg}$. from the distillate.

¥ Instead of 194.93 , as found for "E." 
did at the time; any other value which one could reasonably substitute would lead to, essentially, the same conclusion)-

$$
\mathrm{PtCl}_{4}\left\{\begin{array}{l}
1.925 \mathrm{KCl} \\
0.075 \mathrm{HCl}
\end{array}\right\}+0.482 \mathrm{HCl} .
$$

To check our work, we added up the several instalments of platinum, \&c. found in " $\mathrm{C}$," "E," and "D," and contrasted them with the quantities found in the original chloroplatinate. We had for

\begin{tabular}{|c|c|c|c|}
\hline$" \mathrm{C} "+" \mathrm{E} "+\mathrm{D}, "$ & $\begin{array}{c}\text { Platinum. } \\
2 \cdot 4965\end{array}$ & $\begin{array}{c}\text { Fixed Chlorine. } \\
0.90419\end{array}$ & $\begin{array}{l}\text { Loose Chlorine. } \\
1.79265 \text { grms. }\end{array}$ \\
\hline Original precipitate, & $2 \cdot 4961$ & 0.90038 & 1.79623 \\
\hline
\end{tabular}

Considering the complexity of the operations involved in obtaining the upper set of numbers, the agreement is very satisfactory. From our analysis of chloroplatinates $\mathrm{V}$. and $\mathrm{V} a$., and of their derivatives, we see that in both cases the original chloroplatinate, by being recrystallised, lost chlorine and platinum, with formation of free hydrochloric and chloroplatinic acids, which passed into solution, and the most plausible explanation of the result is to assume that the original chloroplatinates were mixtures of the constitution

$$
\mathrm{PtCl}_{6} \mathrm{~K}_{2}+x\left(\mathrm{Pt}(\mathrm{OH})_{6} \mathrm{H}_{2} \text { or } \mathrm{PtO}_{3} \mathrm{H}_{2}\right) \text {. }
$$

and that part of the hydrochloric acid formed in the substitution of oxygen or hydroxyl for $\mathrm{PtCl}_{4}$-chlorine served to dissolve away the $x \mathrm{PtO}_{3} \mathrm{H}_{2}$ of oxyplatinate of hydrogen as chloroplatinic acid.

Now, in the original chloroplatinate of Experiment V. the weight of platinum " $\mathrm{Pt}$ " per $2 \mathrm{KCl}$ parts was (by synthesis) $=196.67$; while in the twice recrystallised salt the corresponding quantity was $=195.54$.

In the case of chloroplatinate $\mathrm{V} a$. we had " $\mathrm{Pt} "=196.58$, and for its derivatives-

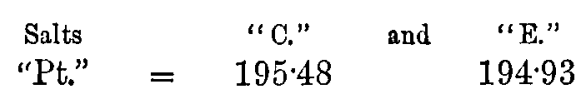

The analysis " $E$ " was carried out on a relatively small scale. The mean of the other two values is 195.51 ; and, supposing our theory to be correct, the true atomic weight of platinum should be either equal to or less than 19551 . Now the degree of completeness with which the surplus platinum is eliminated by recrystallisation should be the higher the purer the chloroplatinate started with, and of all our chloroplatinates those produced in Experiments VIII. and IX. by means of a large excess of chloroplatinic acid apparently come nearest to the ideal substance; hence we thought the best thing we could do would be to prepare a large supply of such chloroplatinate, to analyse it, and then to see what value would come out for the weight of platinum per $2 \mathrm{KCl}$ parts; after recrystallisation from water and hydrochloric acid respectively, which latter solvent we hoped would eliminate the surplus platinum more completely, 
and resubstitute chlorine for the oxychloride oxygen. This programme was carried out in the following experiments :-

\section{Experiment X.}

In each of two parallel experiments, I. and II., * a known weight (about 3.8 grms.) of pure chloride of potassium was dissolved in 50 c.c. of water, and the solution mixed with 150 c.c. of chloroplatinic acid solution, made from Matthey's metal by means of the chlorine process. This reagent contained $0.05 \mathrm{grm}$. of metal per c.c.; hence the excess of platinum used amounted to about 2.5 grms. The mixture was evaporated down on a water-bath, as far as possible, on stirring, the residue mixed with a little water, and re-evaporated. After cooling, 25 c.c. of water were added to produce a "ten per cent" platinum solution, and the mixture allowed to stand cold, in the case of "I." for an hour, in the case of "II." for some 12 hours, with occasional stirring. The precipitate was then thrown on a filter, the basin rinsed with 3 c.c. of the 5 per cent. platinum solution, and the precipitate, which was now all on the filter, washed with other 3 c.c. of the same reagent. After the liquor had drained off, the precipitate was washed exhaustively with alcohol of 95 per cent. (by weight). It was then dried in the filter at $100^{\circ}$, the bulk transferred to a tared glassstoppered cylinder, and in it dried exhaustively at $100^{\circ} \mathrm{C}$. The small remnant on the filter was dissolved off with hot water, evaporated in a tared crucible, and weighed by itself.

The aqueous and alcoholic washings (after removal of the alcohol by distillation from the latter) were reduced with hydrogen in the wet-way, the platinum was filtered off, and the filtrate evaporated to dryness to recover the potassium which had escaped precipitation. This potassium was determined as chloroplatinate (Fresenius' modus).

The results, so far, were as follows:-

\begin{tabular}{|c|c|c|}
\hline Chloride of potassium taken, & $\begin{array}{c}\text { I } \\
3 \cdot 8062\end{array}$ & II. \\
\hline " $\quad$ left unprecipitated, . & 0.0319 & 0.0302 \\
\hline in the chloroplatinate, & $3 \cdot 7743$ & $3 \cdot 7759=\mathrm{A}_{0}$ \\
\hline $\begin{array}{l}\text { Chloroplatinate obtained, } \\
\text { Hence } A_{0}: C \text {, }\end{array}$ & $\begin{array}{c}12 \cdot 3807 \\
0 \cdot 30485\end{array}$ & $\begin{array}{l}12 \cdot 3691=\mathrm{C} . \\
0.30525\end{array}$ \\
\hline
\end{tabular}

which numbers seem to show that chloroplatinate "II." having been lixiviated with chloroplatinic acid for 12 hours (instead of for one hour, like "I."), was purer than No. "I." Each of the two chloroplatinates was divided into three parts: one (A) for the determination of the platinum and total chlorine, another (B) for the determination of the water by the direct method, and a third (C) for

* This second experiment was carried out for us by Mr JAMEs Rossos. 
CRITICAL EXPERIMENTS FOR DETERMINATION OF POTASSIUM, ETC. 585

recrystallisation work. The water-determinations (carried out with $2 \cdot 2$ and $2 \cdot 4$ grms. of substance in I. and II. respectively) gave the following results :-

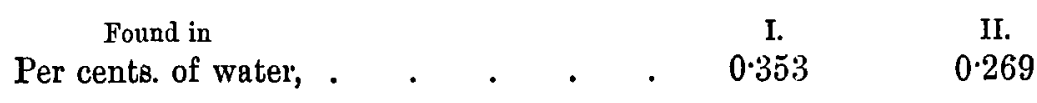

The analyses were made with about 2.4 and 2.7 grms. of substance, dried at $100^{\circ}$. Their results, when combined with the synthetical results for the fixed chlorine, and reduced to $\mathrm{Cl}_{2}=70.91$ parts of the latter, were as follows :-

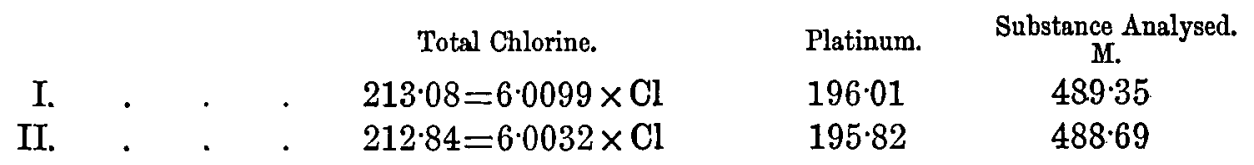

Neglecting the small excesses of chlorine found, we have for

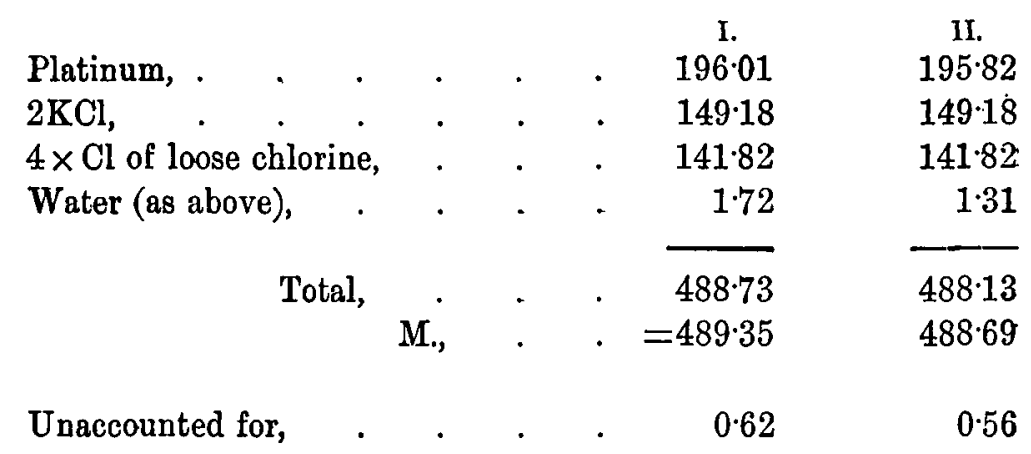

These deficits might be allowed to pass as the cumulative effects of observational errors; but possibly they may be owing to an element of uncertainty in the above numbers for the quantities of water. The samples " $B$," after having been weighed out for the determination of their water, were kept within their weighing-tubes, ${ }^{*}$ over oil of vitriol, for a considerable time, before these determinations came to be carried out. On reweighing the weighing tubes and contents, they were found to have lost ("I".) 1.3 and ("II.") $2 \cdot 1 \mathrm{mgs}$. Assuming these losses to have been suffered merely by the tubes and boats, the percentages of water come out as above reported. Assuming them to have been suffered by the chloroplatinates, their percentages of water rise to ("I. ") $=0.412$, and ("II.") $=0.356$. The corresponding weights per M parts of chloroplatinate then are-

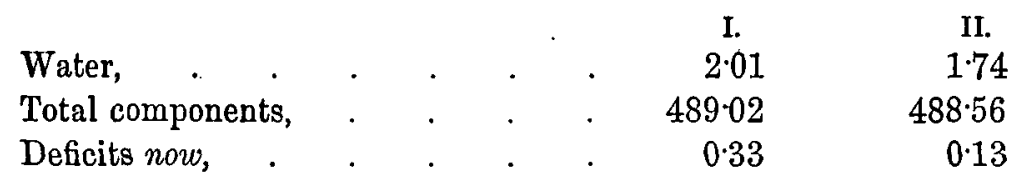

which is more satisfactory.

* Made each out of two lipless test-tubes sliding over one another.

VOL. XXXIII. PART II. 


\section{Recrystallisations.}

The parts "C," amounting conjointly to about $15 \cdot 0$ grms., were dissolved separately, each in 600 c.c. of boiling water, the solutions cooled down, finally in ice, the mother-liquors decanted and filtered off, and the crystals redissolved and recrystallised as before. The two twice-crystallised products were then united, dissolved in hot water, and the solution filtered, to make sure of the absence of every trace of dust in the final product. The filtered solution was evaporated over a water-bath, until crystals had abundantly separated out in the heat, then cooled down, finally in ice, the crystals collected, washed, first with a little cold water, and then exhaustively with absolute alcohol ; the latter washings being collected by themselves. The crystals were dried over oil of vitriol ; they amounted to 4.8 grms., showing that some 10 grms. of salt were contained in the several mother-liquors. The mother-liquors from the first and second crystallisations, when evaporated over a water-bath, gave off towards the end vapours of hydrochloric acid, showing that abundance of chlorine must have been eliminated, and its place taken by oxygen or hydroxyl. The whole of the liquors (minus a small quantity which had been used for these and other tests) were evaporated to dryness over a water-bath, and the residue washed with 91 per cent. (by weight) alcohol. The alcoholic liquors amounted to about 35 c.c.; they exhibited a faint yellow colour, indicating presence of chloroplatinic acid. $2.5 \mathrm{mgs}$. of platinum as chloroplatinic acid, when added to the same volume of alcohol, produced the same intensity of colour; hence (it would appear) very little platinum had been eliminated by the recrystallisations as chloroplatinic acid, or to speak more correctly, the $\mathrm{PtCl}_{6} \mathrm{H}_{2}$ and the $\mathrm{KCl}$ in the (acid) mother-liquor were almost equally balanced, representing just so much $\mathrm{PtCl}_{6} \mathrm{~K}_{2}$ dissolved in hydrochloric acid.

The chloroplatinate thus recovered was dried, recrystallised from water, the crystals mixed with the above 4.8 grms. of recrystallised salt, and the two again recrystallised conjointly. The crystals were washed, first with water, then exhaustively with 91 per cent. alcohol, dried over a water-bath, and preserved in a glass-stoppered bottle as "Recrystallised precipitate." Our mode of procedure may appear irrational to some of our readers, and so it is, in a sense. Our original programme was, starting from the chloroplatinate of Experiment $\mathrm{X}$. to recrystallise it, and analyse both the crystals and the mother-liquor; then to recrystallise the crystals, and analyse the second mother-liquor; and so on, until the platinum per $2 \mathrm{KCl}$ would become constant, but we had not sufficient material for carrying out this scheme.

The aqueous mother-liquors obtained were mixed with 10 c.c. of 20 per cent. hydrochloric acid, evaporated over a water-bath to about 70 c.c., the liquor cooled down in ice, the crystals collected, washed first with 4 per cent. 
hydrochloric acid, then with water, and lastly and exhaustively with the 91 per cent. alcohol. They were dried, transferred to a glass-stoppered tube, and kept as "Precipitate recrystallised from hydrochloric acid." In order to see whether the chloroplatinic acid and the chloride of potassium were still balanced against each other in the hydrochloric mother-liquor, this liquor was evaporated down on a water-bath to about 10 c.c., and mixed, after cooling, with 71 c.c. of 91 per cent. alcohol, to bring down the chloroplatinate (and chloride of potassium, if present). The precipitate, amounting to about 0.66 grm., after having been washed with 91 per cent. alcohol, was extracted, very cautiously, with small successive instalments of ice-cold water. The aqueous washings were evaporated to dryness, the residues again taken up in a little water, filtered, and again evaporated to dryness. This last residue weighed 1.3 mgs., and consisted partly of free chloride of potassium; it gave a precipitate with added chloroplatinic acid. Possibly the alcoholic liquors may have contained more free chloride of potassium, but we unfortunately forgot to examine them.

\section{Analysis of the Chloroplatinate recrystallised from Water.}

This preparation was divided into two parts (amounting to about 2.8 and 3.4 grms.), and then analysed separately.

Found per $2 \mathrm{KCl}$ parts-

$\begin{array}{llcccc} & & & \text { "Chloroplatinate." } & \text { Platinum. } & \text { Loose Chlorine. } \\ \text { I. } & . & . & 484.62 & 195.50 & 138.55=3.9077 \times \mathrm{Cl} \\ \text { II. } . & . & . & 484.91 & 195.69 & 138.66=3.91077 \times \mathrm{Cl} \\ \text { Mean, } & . & . & 484.77 & 195.60 & 138.60=3.9092 \times \mathrm{Cl}\end{array}$

Substituting $0.0908 \times 17$ of hydroxyl for the chlorine deficit, we have (Mean of I. and II.)-

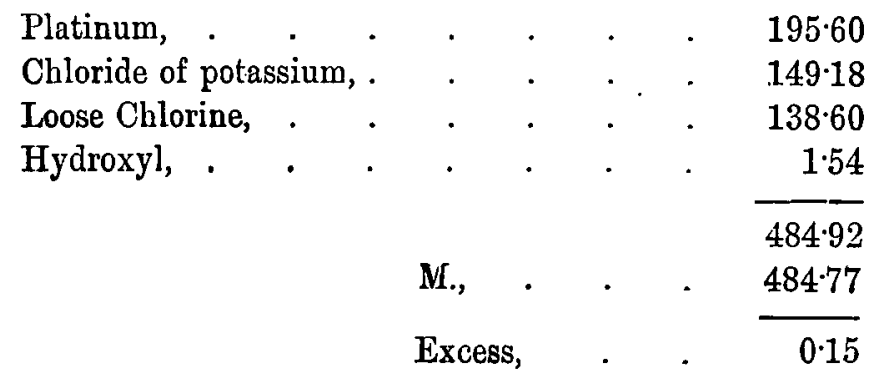

XII. Analysis of the Chloroplatinate recrystallised from Hydrochloric Acid (by $\mathrm{Mr}$ Robson).

This preparation was also analysed twice (substance used $=1.9379$ and 2.3525 grms.); but unfortunately, the determination of the fixed chlorine in the 
second analysis miscarried. Reducing to unit weight of chloroplatinate analysed we had-

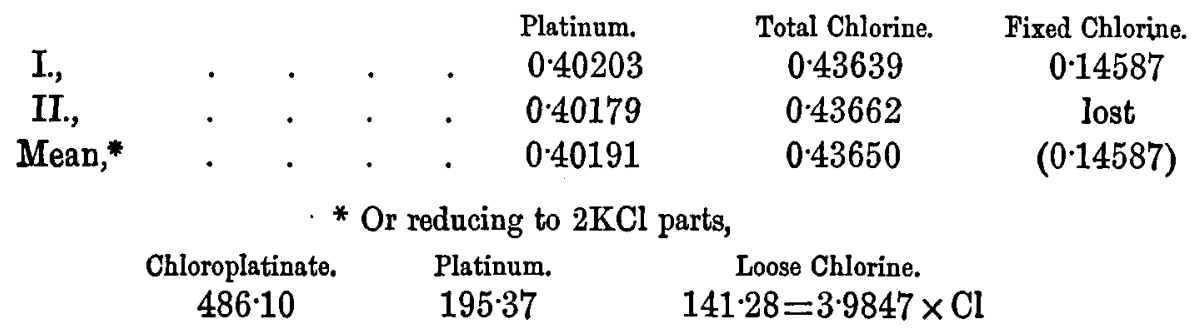

The water was determined directly in 3.2310 grms. : found 5.9 mgs., or 0.1826 per cent., or 0.888 parts per $\mathrm{M}$ parts of chloroplatinate. Substituting $0.01534 \times 17$ of hydroxyl for the chlorine deficit, we have-

$$
\begin{aligned}
& \text { Platinum, . . . } 19537 \\
& \mathrm{~K}_{2} \mathrm{Cl}_{2} \text {, . . . . } 149 \cdot 18 \\
& 3.98466 \times \mathrm{Cl} \text {, . . . } 141.28 \\
& \left\{0.01534 \times \frac{1}{2} 0, . \quad . \quad 0.12\right. \\
& \left\{0.01534 \times \frac{1}{2} \mathrm{H}_{2} \mathrm{O}, \quad . \quad . \quad 0.14\right. \\
& \text { Other water, by analysis, . } \quad 0.75\left\{\begin{array}{c}
0.888-0.138= \\
0.750
\end{array}\right. \\
& \text { Total, . . } \overline{486.84} \\
& \text { M., . }=486 \cdot 10 \\
& \text { Excess of analysis, }=\overline{0.74}
\end{aligned}
$$

\begin{tabular}{|c|c|c|c|c|}
\hline Experiments. & P.* & $\begin{array}{l}\text { Volume of mixture of Reagents } \\
\text { per } 1 \mathrm{grm} \text {. of } \mathrm{KCl} \text { used. }\end{array}$ & Pt. & \\
\hline I. & $209 ?$ & Mixture evaporated to small & $\int 196 \cdot 28$ & \\
\hline II. & $218\}$ & volume, then alcohol added, & $\{195 \cdot 67$ & \\
\hline III. & 206 & 32 c.c. & $195 \cdot 67$ & Mean \\
\hline IV & 214 & $32 n$ & $195 \cdot 79$ & $\mathbf{P t}=$ \\
\hline V. & 195 & $34 »$ & $196 \cdot 64$ & $196 \cdot 23$ \\
\hline $\mathrm{V} a$. & 203 & 50 & 196.58 & \\
\hline VI. & 196 & (Precipitated by alcohol.) & $196 \cdot 95$ & \\
\hline
\end{tabular}

which is not too much.

It is surprising to see that the action of the hydrochloric acid did not resubstitute $\mathrm{Cl}$ for all the $(\mathrm{OH})$ of the recrystallised salt.

The following table summarises what we found regarding the quantity of platinum ("Pt") present, per $2 \mathrm{KCl}$ parts, in our several chloroplatinates :-

1. Chloroplatinates prepared by Simple Precipitation; Platinum moderately (if at all) in excess.

* "P" stands for weight of platinum used as chloroplatinic acid, per $2 \mathrm{KCl}$ parts. 
CRITICAL EXPERIMENTS FOR DETERMINATION OF POTASSIUM, ETC. 589

2. Chloroplatinate prepared by precipitating Chloroplatinic Acid with a large excess of Chloride of Potassium.

$\begin{array}{ccc}\text { Experiment. } & \text { P. } & \begin{array}{c}\text { Volume of mixture of Reagents } \\ \text { per } 1 \text { grm. of KCl used. }\end{array} \\ \text { VII. } & 40 & 53 \cdot 7 \text { c.c.* }\end{array} \quad\left\{\begin{array}{c}\begin{array}{c}194.65 \text { (196.78 per } \\ 4 \mathrm{Cl} \text { parts of loose } \\ \text { chlorine). }\end{array} \\ \text { (See context.) }\end{array}\right.$

3. Chloroplatinate made by precipitating Chloride of Potassium with a large excess of Chloroplatinic Acid.

$\begin{array}{cccc}\text { Experiment. } & \text { P. } & \begin{array}{c}\text { Volume of mixture of Reagents } \\ \text { per } 1 \text { grm. of KCl used. }\end{array} & \text { Pt. } \\ \text { VIII. } & 294 & 160 \text { c.c. } & 195 \cdot 87\end{array}$

4. Chloroplatinates made from Chloride of Potassium, by cvaporating with a large excess of Chloroplatinic Acid, washing with first 5 per cent. Platinum Solution, and lastly with Alcohol.

$\left.\begin{array}{cccc}\text { Experiments. } & \text { P. } & \begin{array}{c}\text { Volume of mixture of Reagents } \\ \text { per 1 grm. of KCl used. }\end{array} & \text { Pt. } \\ \text { IX. } & 294 & \text { (Evaporation), } & 195.59 \\ \text { X. (I.) } & 294 & " & 196.01 \\ \text { X. (II.) } & 294 & " & 195 \cdot 82\end{array}\right\} \begin{gathered}\text { Mean= } \\ \text { 195.81 }\end{gathered}$

5. Recrystallised Chloroplatinates.

Supplementary Experiment to V., . . . . . . . . 19554

" " to Va., . . . . . . . 19548 Mean=

Experiment XI., (Recrystallisation of X. from water), . . $\quad .195 .60\} 195.50$

" XII., (Recrystallisation from dilute hydrochloric acid), . 195.37

From our recrystallisation experiments (if our theory be correct), it follows that the true atomic weight of platinum (apart from observational errors) lies at, or is less than, 195.50 .

This number, $195 \cdot 50$, then, affords an upper limit to the unknown number Pt. Seubert's experiments enable us to find a lower limit.

Seubert, in his Memoir, gives the results of two analyses of one, and of six of another, preparation of chloroplatinate of potassium. The chloride of potassium and the platinum were determined in each case, the loose chlorine only three times. His mean results, referred to 100 parts of substance analysed, were as follows:-(Mean relative uncertainty means the mean deviation from the mean, measured by the mean itself; or the uncertainty per unit of the quantity determined).

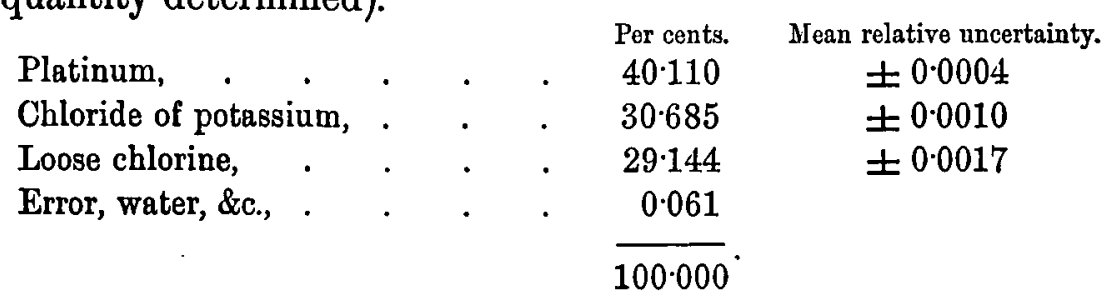

* Per 1 grm. chloride of potassium as calculated from the platinum, assuming $\mathrm{Pt}$ to correspond to $2 \mathrm{KCl}$. 
Reducing to $2 \mathrm{KCl}$ parts, we have

$\begin{array}{cccc}2 \mathrm{KCl} . & \text { Platinum. } & \text { Loose Chlorine. } & \text { Chloroplatinate as analysed. } \\ 149 \cdot 18 & 195 \cdot 00 \pm 27 & 141 \cdot 688 & 486 \cdot 17 \\ & & \text { or } 3.9963 \times \mathrm{Cl} \pm .011 \mathrm{Cl} & \end{array}$

But Seubert's chloroplatinate was prepared by precipitating a (rather dilute, ice-cold) solution of chloroplatinic acid, with, in two cases, 1.33 times, in the other six cases, twice, the calculated weight of chloride of potassium. His precipitate, therefore, in all probability, contained loosely combined (surplus) chloride of potassium.

Side by side with the potassium salt, Seubert prepared ammonium chloroplatinate by a closely similar process ; the latter he recrystallised to remove "niedergerissenen salmiak"; the former he accepted as normal, although it also suffered a loss of alkyl chloride on recrystallisation; but in this case, it appears, Seubert assumes that the eliminated $\mathrm{KCl}$ came out of the chloroplatinate itself. For this, we submit, he had no excuse. Our view of the matter is that both his chloroplatinates contained niedergerissenes alkylchloride. In the case of his chloroplatinate of potassium, a very little surplus chloride of potassium was sufficient to make his value $\mathrm{Pt}$ by half a unit too low. In order to see by how much we would have to correct down his proportion of $\mathrm{KCl}$ in his chloroplatinate, let us refer his numbers to $\mathrm{Pt}=195.50$; they then read as follows :-

$\begin{array}{ccc}\text { Chloride of Potassium. } & \text { Platinum. } & \text { Loose Chlorine. } \\ 149.562 \pm 0.21 & (195.50) & (4.0065 \pm 0.0084) \mathrm{Cl}\end{array}$

for which we may substitute, without correcting by more than the mean errors

$$
\begin{array}{lll}
149.35 & (195.50) & 4.000 \times \mathrm{Cl}
\end{array}
$$

Now Seuber's's chloroplatinate of potassium, from the way in which it was prepared, was bound to contain some "niedergerissenes" chloride of potassium; that its proportion should have amounted to less than 0.00114 of the chloride of potassium of the real chloroplatinate in his precipitate, is not at all likely; hence we are justified in concluding that his analyses of chloroplatinate of potassium fall in better with our $\mathrm{Pt}=195.5$, than with his own $194 \cdot 8$.

A critique of his analysis of the ammonium salt leads to a similar result. The results of these analyses may be summarised as follows:-Found for "Pt" (referred to $\mathrm{O}=16$ ).

1. By determining the weight-ratio of platinum to non-platinum, in a salt precipitated from sal-ammoniac solution, by an excess of chloroplatinic acid (Darstellung I.), . . . . . . . . • . .

2. By determining the same ratio in a salt obtained with an excess of salammoniac (and not recrystallised) (Darstellung II.), . . . . . . . 19453 
3. By determining the same ratio in a salt prepared from salt of Darstellung II.

4. By determining the same ratio in a salt prepared from chloroplatinic acid by addition of about 2.5 times the calculated weight of sal-ammoniac, washing,

and recrystallising the precipitate (Darstellung IV.), .
5. By determining the weight-ratio of platinum to total chlorine in three preparations, namely :-

(a) One of Darstelling II. not recrystallised.

(b) One of Darstellung III. (precipitation of chloroplatinic acid, prepared by means of chlorine gas and hydrochloric acid, with a somewhat considerable excess of sal-ammoniac).

(c) One of Darstellung IV.

The values Pt (calculated by us from his numbers for platinum and total chlorine) were as follows :-

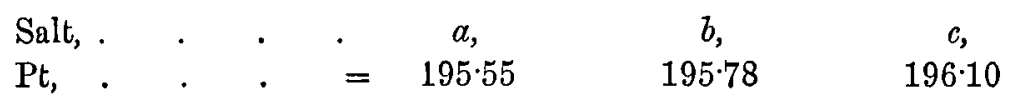

Now, all these chloroplatinates were liable to contain water. Those which were not recrystallised,-excepting (1),-were almost bound to contain free sal-ammoniac; those which were, probably contained $\mathrm{OH}$ instead of part of their $\mathrm{Cl}$. In the case of determinations (5), $(a),(b),(c)$, however, the presence of water does not tell upon the results; hence, if we had only these three analyses to go by, we should say, results $(a)$ and $(b)$ are probably too low, because (free sal-ammoniac) chlorine was determined as chloroplatinate-chlorine; (c) is probably too high, because the salt contained oxygen in place of part of its chlorine, and we should take the mean of the mean of $(a)$ and $(b)$, and of (c) as the most probable value, and put down $\mathrm{Pt}=195 \cdot 89 \pm 0 \cdot 22$.

Of result (1) it is difficult to say whether it is more likely to be too high or too low, because it may have contained surplus platinum; we must accept the $195 \cdot 18$ as it stands.

Result (2) is sure to be too low, because it must be presumed to have contained both water and surplus sal-ammoniac. Hence the value 194.53 is less than the true Pt.

Preparations (3) and (4) were probably free of surplus sal-ammoniac or platinum; but they nay have contained water, which would make the resulting $\mathrm{Pt}$ too low; and they probably contained hydroxyl in place of their chlorine, which would tend the opposite way. But result (3) is derived from only one analysis. Hence (as (2) is out of court), the most reasonable mode of utilising the determinations (1) to (4), is to take the mean of (1) and (4); or rather, as (1) included six and (4) included nine analyses, to take $\mathrm{Pt}=(6 \times 195 \cdot 18+9 \times 195.50) \div 15=195 \cdot 37$, and assuming this to have, say, 10 times the "weight" of the result deduced from analyses (5), we have, finally, $\mathrm{Pt}=195 \cdot 42$, which number falls in well enough with SEUBERT's analyses of 
chloroplatinate of potassium, and may be adopted as being virtually his final result. Combining it with our own number 195.50 , we have $\mathrm{Pt}=195.46$, or rather 195.5 (because the uncertainity on either side is more than \pm 0.1 ), as being at present the most probable value of the constant.

The true number, we mean a number ranking in probable precision with, say, Marignac's number for chlorine, will, we hope, be determined one day, but if so, it must be derived from other experiments than analyses of chloroplatinates, which are clearly unfit for the purpose.

If, instead of searching for the true atomic weight, we want the quasi "constant," which tells us how much platinum is associated with $2 \mathrm{KCl}$ parts of chloride of potassium, in a chloroplatinate produced in the ordinary methods of analysis, even our value is too low by about half a unit. So at least we must conclude from our experiments on Finkener's and on Tatrock's form of the chloroplatinate process for the determination of potassium.

These experiments are detailed in the next following section.

\section{Finkenel's and Tatlock's Methods of Potash Determination.}

Finkener's Method.

This method is not so widely known in this country as it ought to be, we therefore begin by shortly explaining it. Assuming, for the sake of greater definiteness, that the substance to be analysed is a mixture of chlorides and sulphates of potassium, sodium, and magnesium, it is dissolved in water, and the solution mixed with a quantity of sulphuric acid sure to be sufficient for converting all the foreign oxides into sulphates; a quantity of platinum solution, a little more than equivalent to the potassium to be determined is now added, and, if necessary, so much water that the chloroplatinate precipitate produced is dissolved in the next operation, which is to heat the mixture on a boiling water bath. The solution produced is evaporated on a water-bath to the consistence (after cooling) of a magma. This is allowed to cool, mixed with ether-alcohol,"* and allowed to stand, well covered, until the precipitate has settled completely. The precipitate, - a mixture of chloroplatinate of potassium and the sulphates of the foreign metals,-is washed with ether-alcohol, to be worked up in one or other of the following two ways:-(A) The precipitate is heated in hydrogen gas to dull redness, so as to reduce the chloroplatinate to $\mathrm{Pt}+2 \mathrm{KCl}$, the product treated with water (then with hydrochloric acid, if necessary, and again with water); the residual platinum ignited and weighed, to be calculated into potassium. (B) The precipitate is lixiviated, as quickly as possible, with concentrated (cold) solution of sal-ammoniac, until the filtrate is

\footnotetext{
* "Ether alcohol," in connection with Finkeser's method, always means 1 volume of anhydrous
} ether and 2 volumes of absolute alcohol. 
free of sulphate; the residue (chloroplatinate of potassium plus sal-ammoniac). is dried, ignited in hydrogen, the platinum collected as before, and weighed. In this case, of course we have the option of determining the chloride of potassium extracted by water from the ignited residue, either quite directly by evaporation, \&c., or indirectly by determining its chlorine.

When one of us, some years ago, was commissioned by the Challenger Authorities to carry out exact analyses of a large number of samples of oceanwater, he inquired critically into the several known methods which might have been used for the determination of the small proportion of potassium present in sea-water salts, and found that FinkEner's was the only one which afforded a fair approximation to the truth. To render his results susceptible of subsequent correction, he brought the Finkener method for his purpose into a definite form, regarding which it may suffice here to state the main points as follows :-*

100 c.c. of sea-water are weighed, evaporated to near dryness, and the salts made into normal sulphates; these are dissolved in water, any sulphate of lime, \&c., is filtered off, and in the filtrate, the potassium is determined by Finkener's method (form A) by means of a quantity of chloroplatinic acid containing $200 \mathrm{mgs}$. of platinum, i.e., about twice the calculated quantity.

A number of analyses of synthetically prepared mixtures showed that the platinum-weight, when multiplied by $\mathrm{K}_{2} \mathrm{O} \div \mathrm{Pt}=94 \div 198=0.4747$, gave results about 1 per cent. short of the potash used (as chloride of potassium). When the factor, calculated from SEUBERT's atomic weight for platinum $(\mathrm{Pt}=194.8)$ and STAS' value for $\mathrm{K}_{2} \mathrm{O}$ was nsed (i.e., the factor 0.48386$)$ t the results were about by 0.01 of their value too high. We may state, in passing, that it was this observation which gave the start to the experiments reported above as Nos. I. to VII. in the First Series.

After the "Challenger" analyses had been reported, we again determined, for our own satisfaction, what degree of exactitude would have been attained if we had separated out the potassium and sodium as chlorides (free of calcium and magnesium), and then applied what we are in the habit of calling "Fresenius' Method," because it is the one recommended for mixtures of the two alkyl-chlorides in his handbook of analysis.

Leaving the errors involved in the elimination of the lime and magnesia on one side, we prepared a mixture containing very nearly the weights of chloride of potassium and sodium present in 100 c.c. of ocean-water (that of the former, of course, adjusted exactly), and analysed it by means of the following method :-

*For details, see "Challenger Memoirs," Physics and Chemistry, vol. i. (appendix), p. 233 et seqq.; see also body of Memoir, pp. 12 et seqq.

$\dagger \frac{\text { New factor }}{\text { Old factor }}=1.0192$.

VOL. XXXIII. PART II. 
The mixture is dissolved in a little water, mixed with more of chloroplatinic acid than necessary for the conversion of both metals into chloroplatinates, and evaporated to a magma on a water-bath. The residue, after cooling, is digested in 30 c.c. of alcohol of 80 per cent. by volume, the liquid decanted off through a small filter, and the precipitate washed with the same alcohol, until the last runnings give only an opalescence with nitrate of silver. The washed precipitate is dried on the filter, dissolved off with boiling water, the solution evaporated in a tared crucible to dryness, dried exhaustively at, first $105^{\circ} \mathrm{C}$., then at $130^{\circ}$, and weighed. From the weights, the potassium is calculated $\left(\right.$ as $\left.\mathrm{K}_{2} \mathrm{O}\right)$ in order to see what the exact, but unreasoning, application of the method would lead to.

But the precipitate can neither be presumed to contain the whole of the potassium, nor to be pure; hence-

Firstly, the crude precipitate is reduced in hydrogen, the platinum weighed, and from the aqueous solution of the chloride of potassium, the latter recovered by a renewed application of FresEnIUS' method.

Secondly, the filtrate from the original chloroplatinate (which contains the sodium and excess of chloroplatinic acid), is freed from its alcohol, by evaporation to dryness, the residue reduced in hydrogen, the alkalies are extracted with water, and made into normal sulphates. In these, the potassium which escaped precipitation is recovered by FinkENER's method (sal-ammoniac form). The platinum obtained in the reduction of this small quantity of chloroplatinate of potassium is weighed, and the chloride of potassium reconverted into chloroplatinate by Fresenius' process, to be weighed as such.

The pure chloride of potassium used for preparing the mixtures was made from recrystallised chlorate.* For the preparation of potash-free chloride of sodium, we used two methods, of which the following gave the best results :Ordinary "pure" sulphate of soda is dissolved in water, and the solution saturated with hydrochloric acid gas, to precipitate about one-half of the alkali metal as chloride, which is collected on a funnel stopped up with a round glass bead fitting pretty closely into the neck of the funnel, and washed with fuming hydrochloric acid. The salt is then dried, redissolved in water, and reprecipitated by hydrochloric acid gas. The dried product contained a mere trace of sulphate, which was neglected. To test the salt for potassium, a large quantity of it was made into sulphate, and 23 grms. of this subjected to the Finkener process, sal-ammoniac form. The potassium extracted was determined in the way just described, and its chloroplatinate identified by microscopic inspection. It amounted to $0.38 \mathrm{mg}$. of $\mathrm{K}_{2} \mathrm{O}$ calculated from the platinum, and to $0.43 \mathrm{mg}$. calculated from the chloroplatinate. From the mean, $0.40 \mathrm{mg}$., we

* We subsequently came to prefer the perchlorate for obvious reasons. 
calculated that 100 grms. of the original chloride of sodium contained $2 \cdot 1$ mgs. of potassium calculated as $\mathrm{K}_{2} \mathrm{O}$. This potassium was allowed for in the test experiments.

Two such experiments were made, and both are reported in the "Challenger Memoir," along with some further details which are here omitted for brevity's sake. We satisfy ourselves with quoting one of the two reports.

\begin{tabular}{|c|c|c|}
\hline Chloride of sodium used, . $\quad$ • $\quad$. . & & rms. \\
\hline Potassium preser & $\begin{array}{l}\text { Old Atomic } \\
\text { Weights. }\end{array}$ & $\begin{array}{c}\text { New Atomic } \\
\text { Weights. }\end{array}$ \\
\hline Chloride of potassium taken, & $50 \cdot 04$ & $50 \cdot 12$ \\
\hline$" \quad$ in the chloride of sodium, & 0.06 & 0.06 \\
\hline & $50 \cdot 10$ & $50 \cdot 18$ \\
\hline
\end{tabular}

Analysis.-Potassium found, calculated as $\mathrm{K}_{2} \mathrm{O}$ in milligrammes.

I. In the crude chloroplatinate by calculation, . $\quad 47.90$

$48 \cdot 35$

Ia. By calculation from the metallic $\mathrm{Pt}$ obtained therefrom, . . . . . . . . $47.62 \quad 48.53$

II. In the pure chloroplatinate, . . . . $47.06 \quad 47.50$

II $a$. Calculating from the metallic Pt obtained, . $\quad$. $\quad 46.62 \quad 47.51$

III. In filtrate from crude chloroplatinate, . . $\quad 264 \quad 268$

IV. In filtrate from pure chloroplatinate, . . . . $\quad 0.85 \quad 0.87$

Sum of II., III., and IV.,. $\quad$. . . . . . $50.55 \quad 51.05$

Excess over synthesis, . $\quad . \quad . \quad . \quad . \quad 0.45 \quad 0.87$

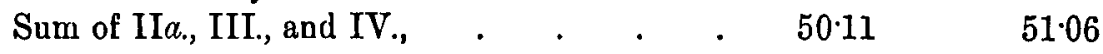

Excess over synthesis, . $\quad . \quad . \quad . \quad . \quad 0.01 \quad 0.88$

From the numbers given under I. and the synthesis we see that FRESENiUS' method, if used as it stands, would $\begin{array}{llll}\text { have given a loss of potash amounting to . . . } & \text {. } & 220 & 1.83\end{array}$

Or, per 100 of $\mathrm{K}_{2} \mathrm{O}$ to be determined, to $\quad$. $\quad$. $\quad$. 444

Note. - The chloroplatinates were weighed after being dried at $130^{\circ}$. The weights, after drying at $105^{\circ}$, were only about 0.001 more per unit weight of precipitate.

For the analysis of salt mixtures poor in potassium Finkener's is the only method that works at all ; for the analysis of potassiferous substances generally, it of course only competes with other methods, but over any of these it offers the great advantage of being in a high degree independent of the nature of the foreign bases present. It must be admitted, however, that the Finkener method, in the form in which it came out of the inventor's hands, owes what there is in it of precision, to some extent, to compensation of errors. Finkener's own test-analyses prove this : they gave very exact results, because Frnkener, in reducing his platinum-weights to potash, used the old atomic

$$
\begin{aligned}
& * \mathrm{~K}=39 ; \mathrm{Cl}=35 \cdot 5 ; \mathrm{Pt}=198 . \\
& \dagger \mathrm{K}=39 \cdot 13 ; \mathrm{Cl}=35 \cdot 454 ; \mathrm{Pt}=194 \cdot 8 .
\end{aligned}
$$


weight of platinum $(\mathrm{Pt}=198)$; if he had used SevBert's value, which no doubt comes nearer to the truth, his results would have been by about 2 per cent. of their value higher (in calculating for $\mathrm{K}_{2} \mathrm{O}$ ).

We thought it worth while to try and ascertain the several errors involved in the method, with the view of either evading them, or finding a formula for their correction.

There is, however, one other form of the platinum process which, although of less general applicability, as far as it goes, would appear to be as independent of the nature of the impurities in the substance analysed as Finkener's. We refer to $\mathrm{Mr}$ TATLOCK's method, which, with anything that falls within the denomination of "potash salt" (pure or impure) is well known to give, to say the least of it, fair results.

We accordingly decided to test both these methods, as far as they compete side by side with each other. Passing over a deal of pioneering work, which, instructive as it was to ourselves, would probably not interest the majority of our readers, we will begin with our experiments on FinkENER's method, and in the first instance detail certain experiments made for determining its value for the determination of relatively minute quantities of potassium salts, diffused throughout a substance consisting chiefly of sulphates and chlorides of sodium and magnesium. Let us state at once that we always worked with mixtures of this kind, because other acid-tests (than $\mathrm{SO}_{4}$ or $\mathrm{Cl}_{2}$ ) are of rare occurrence, and other bases than $\mathrm{MgO}$ and $\mathrm{Na}_{2} \mathrm{O}$ are easily removed by analytical processes. From what we are going to say, every chemist will easily see to what extent the method is more widely applicable,--as a matter of probability at least.

\section{Blank Experiments with Unmixed Sulphate of Soda.}

The sulphate was made from the chloride of sodium which had served for the experiments detailed above, pages 594 and 595. It contained potassium $=1.93 \mathrm{mgs}$. of chloride in 71.08 grms.

Experiment I. $1 \cdot \tilde{5}$ grms. of the sulphate of soda (anhydrous) were dissolved in 30 c.c. of water, the solution mixed with chloroplatinic acid, containing 50 mgs. of metallic platinum, and evaporated to a magma. To it, alcohol (1 vol.) was added, and then ( $\frac{1}{2}$ vol. of) ether, to dissolve out the chloroplatinic acid, which was washed away as far as possible with ether-alcohol. The remaining salt was almost, but not quite white. This was no more than we had expected, having previously found that the mixture obtained in FinkENER's process is liable to contain an excess of platinum. To remove this admixture, the salt, after removal of the ether and alcohol by drying at a gentle heat, was redissolved in water, and "re-Finkenerised" without platinum; i.e., again evaporated to a magma, and the latter washed with ether-alcohol. The platinum thus 
extracted was determined, and found to amount to $0.8 \mathrm{mg}$. The recrystallised* sulphate, when heated in hydrogen gas under a funnel, and (the product) treated with water, left $1.2 \mathrm{mgs}$. of platinum, corresponding to $0.91 \mathrm{mg}$. of chloride of potassium. The $0.04 \mathrm{mg}$. (which had been present in the sulphate used) are not deducted.

Experiment II.-A repetition of I., except that $100 \mathrm{mgs}$. of $\mathrm{H}_{2} \mathrm{SO}_{4}$ in the form of standard acid, were added in the recrystallisation, with the view of decomposing the chloroplatinate of sodium suspected to be present. Platinum obtained from the recrystallised salt, $=0.5 \mathrm{mg} .=0.34 \mathrm{mg}$. of chloride of potassium (corrected for the potassium in the sulphate of soda used).

Experiment III.-Three grammes of sulphate of soda used, and the sulphate obtained recrystallised twice, without addition of sulphuric acid. Residual platinum, $=0.4 \mathrm{mg}$. $=0.30 \mathrm{mg}$. of chloride of potassium, or $0.22 \mathrm{mg}$. after deducting the $0.08 \mathrm{mg}$. really present.

Experiment IV.-A repetition of Experiment III., except that $200 \mathrm{mgs}$. of $\mathrm{H}_{\bar{\varepsilon}} \mathrm{SO}_{4}$ were added in each recrystallisation. Residual platinum exactly the same; i.e., a quantity indicating $0.22 \mathrm{mg}$. of adventitious chloride of potassium.

Experiments III. and IV. were made side by side of each other.

Experiment V.-To study the effect of the added sulphuric acid on chloroplatinate of potassium, $0.2006 \mathrm{grm}$. of pure chloroplatinate of potassium was dissolved in 40 c.c. of boiling water, containing 25 mgs. of $\mathrm{H}_{2} \mathrm{SO}_{4}$, the mixture evaporated to a magma, and Finkenerised. The resulting salt was " recrystallised " again with 25 mgs. of $\mathrm{H}_{2} \mathrm{SO}_{4}$. Both mother-liquors were yellow. They contained, that from the first precipitation $0.9 \mathrm{mg}$., that from the second $0.6 \mathrm{mg}$. of platinum, equal to 0.68 and $0.46 \mathrm{mg}$. of chloride of potassium respectively.

Seeing that sulphuric acid, under the circumstances, decomposes chloroplatinate of potassium appreciably, we tried, in

Experiment VI., the effect of sulphate of lithium on chloroplatinate of potassium, because, supposing it to prove inert towards chloroplatinate of potassium, it would have afforded an admirable reagent for the elimination of foreign chloroplatinates from a FinKENER residue. $0.5 \mathrm{grm}$. of chloroplatinate of potassium when Finkenerised with addition of $0.1 \mathrm{grm}$. of pure sulphate of lithia, yielded a filtrate containing $3.8 \mathrm{mgs}$. of platinum, $=2.9 \mathrm{mgs}$. of chloride of potassium. On " recrystallising" with $50 \mathrm{mgs}$. of the lithia salt, the liquor contained $4.0 \mathrm{mgs}$. of platinum, equal to $3.0 \mathrm{mgs}$. of chloride of potassium.

This shows that sulphate of lithia is not available for the purpose aimed at.

\section{Experiments with Sulphate of Soda, containing added Potassium.}

I. A quantity of sulphate of soda equivalent to 8.73 grms. of chloride $(\mathrm{NaCl})$, and a known weight of a standard solution of chloride of potassium

* We adopted this word for designating the operation described. 
containing $235.5 \mathrm{mgs}$. of $\mathrm{KCl}$, were dissolved in hot water, and Finkenerised with a quantity of platinum solution containing $600 \mathrm{mgs}$. of platinum, i.e. about twice the quantity demanded by the potassium present. The magma obtained on evaporation was mixed with 50 c.c. of absolute alcohol, and allowed to stand for three quarters of an hour ; 25 c.c. of ether were then added, the precipitate well pounded up with a pestle, and the whole allowed to stand under a small bell-jar for two hours. The mother-liquor was then decanted off through a filter, and the precipitate washed with ether-alcohol (2 volumes of alcohol and 1 volume of ether), until the last runnings gave only an opalescence with nitrate of silver. The alcohol-ether filtrate was kept as "I."

From the precipitate the sulphates were extracted by lixiviation with (140 grammes of) saturated sal-ammoniac solution. The last runnings gave only a slight turbidity with chloride of barium.

The solution obtained was put aside as "II."

The remaining chloroplatinate was dissolved in hot water and in the solution, reduced by hydrogen. The platinum obtained was filtered off and weighed; it amounted to $311.0 \mathrm{mgs}$. The filtrate from the metallic platinum was evaporated to dryness, the residue ignited to drive off the ammonia-salt, and the resulting crude chloride of potassium weighed; it amounted to $241.5 \mathrm{mgs}$. This salt was dissolved in water, and its potassium eliminated and weighed as chloroplatinate, by a method which was essentially TATLOCK's, (extraction of foreign matter from the evaporated residue by 5 per cent.* chloride of platinum solution, and washing finally with strong alcohol). From the alcoholic washings the alcohol was distilled off, the residue united with the aqueous washings and filtrates, and from the mixture the platinum eliminated by hydrogen in the wet way. The solution was weighed and fractionated for the determination of the sulphuric acid, and of the total bases as sulphates.

The following is a summary of the results, stated in milligrammes :-

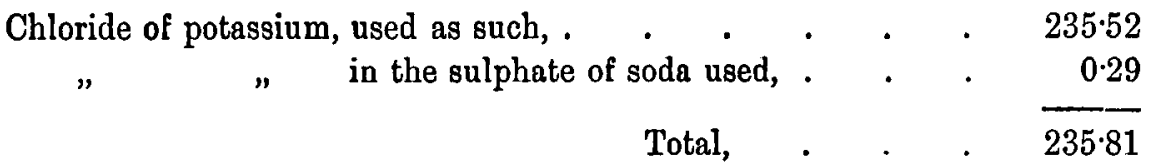

Chloride of Potassium obtained.

A. (Apparently) in the crude chloroplatinate, as calculated from the $\mathrm{Pt}$ (factor $=\cdot 76117$,

Excess found, . . . . . . . . . . . . . 0.29

B. In the alcohol-ether filtrate and washings; determined as $\mathrm{PtCl}_{6} \mathrm{~K}_{2}$. Weight calculated from this precipitate $=0.61 \mathrm{mg}$; from the platinum eliminated from it by $\mathrm{H}_{2}=0.84 \mathrm{mg}$; mean, . . . . . . . . . 0.73

* Meaning a solution containing 5 centigrms. of platinum metal per c.c. The phrases " 5 per cent.," " 10 per cent.," \&c., platinum solution must be read in this sense. 
C. From the sal-ammoniac liquor ("II."); determined in ignited residue as $\mathrm{PtCl}_{6} \mathrm{~K}_{2}$, and as $\mathrm{Pt}$ climinated from the same. Weight calculated from the $\mathrm{PtCl}_{6} \mathrm{~K}_{2}$ was 1.22 ; from the $\mathrm{Pt}$ it was $1.60 \mathrm{mgs}$; mean, 1.41 ; corrected for the $\mathrm{KCl}$ contained in the sal-ammoniac used,* . . . . . .

D. The crude chloride of potassium, extracted from the crude chloroplatinate, weighed $241.5 \mathrm{mgs}$. According to the analysis of the filtrate from the $\mathrm{PtCl}_{6} \mathrm{~K}_{2}$ obtained from it, it contained $6.57 \mathrm{mgs}$. of $\mathrm{Na}_{2} \mathrm{SO}_{4}$; deducting this we have-

F. Pure chloride of potassium,
The pure chloroplatinate of potassium obtained from the crude chloride of potassium was weighed; its platinum extracted by $\mathrm{H}_{2}$ in the wet-way, and weighed likewise.

$\mathrm{D}_{0}$. The $\mathrm{KCl}$, calculated

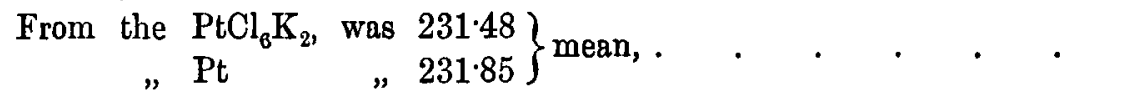

This $\mathrm{D}_{0}$ then was the weight of chloride of potassium which the FinKEner process had actually separated out; the close correctness of the number " $A$ " was owing to the presence of foreign chloroplatinates in what was nominally $\mathrm{PtCl}_{6} \mathrm{~K}_{2}$.

E. In the analysis of the crude chloride of potassium, by means of the platinum
process, the little potassium which escaped precipitation was determined as $\mathrm{PtCl}_{6} \mathrm{~K}_{2}$, and calculated as $\mathrm{KCl}$; found equal to . . .

To check the work, let us add up the various instalments of chloride of potassium found.

$$
\begin{aligned}
& \mathrm{D}_{0}+\mathrm{B}+\mathrm{C}+\mathrm{E}, \quad . \quad . \quad . \quad . \quad . \quad . \quad . \quad \text {. } 2370
\end{aligned}
$$

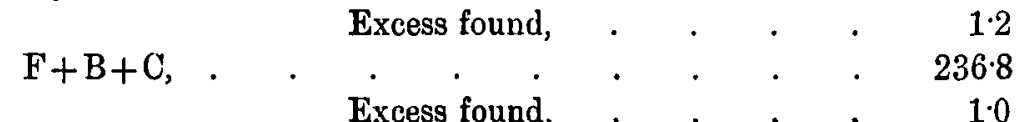

II. A Second Experiment differed from the ("I.") just detailed in the following points:-(To the same quantity of sulphate of soda as had been used in "I.") only 23.86 mgs. of chloride of potassium were added, and the mixed solution Finkenerised with five times the calculated quantity of platinum solution. The salt mixture $\left(x \mathrm{Na}_{2} \mathrm{SO}_{4}+\mathrm{PtCl}_{6} \mathrm{~K}_{2}\right)$, after a final wash with pure ether, was allowed to dry, and "recrystallised" without any addition (of $\mathrm{H}_{2} \mathrm{SO}_{4}, \& \mathrm{c}$.). In the treatment of the "recrystallised" salt mixture $\left(x \mathrm{Na}_{2} \mathrm{SO}_{4}+\mathrm{PtCl}_{6} \mathrm{~K}_{2}\right)$ with sal-ammoniac, an exhaustive extraction of the sulphate was not insisted upon, for not decomposing too much of the chloroplatinate of potassium. The impure residue obtained after treatment with 90 grms. of sal-ammoniac solution was dissolved in hot water, the platinum reduced out by $\mathrm{H}_{2}$ and weighed. The sal-ammoniac liquor was preserved. The results, so far, were as follows (in milligrammes) :-

\footnotetext{
* Determined by a blank carried out on a large scale.
} 
Chloride of potassium used, including $0 \cdot 29 \mathrm{mg}$., from

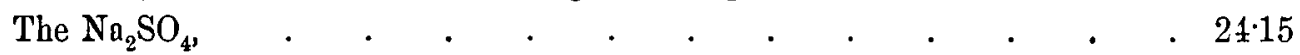

Found.

(Apparently) in the chloroplatinate-mixture, as calculated from the platinum extracted, .

In the second ether-alcohol filtrates, $5 \cdot 8 \mathrm{mgs}$.

Of these $5.8 \mathrm{mgs}$. of platinum, however, 0.8 was present as $\mathrm{PtCl}_{6} \mathrm{~K}_{\mathrm{p}}$, hence the recrystallisation process, besides removing $5 \cdot 8-0 \cdot 8=5.0 \mathrm{mgs}$. of platinum present as chloroplatinate, removed also $0.8 \mathrm{mg}$. of platinum which ought to have remained.

Hence, if recrystallisation had not been resorted to, the chloride of potassium

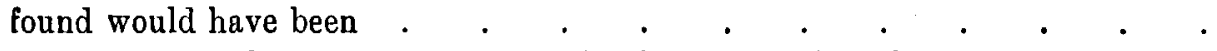

Errors in the actual and imagined determinations $=-4.97$ and -0.56 respectively.

Experiment $\mathrm{II} a$. -The residue $\left(y \mathrm{Na}_{2} \mathrm{SO}_{4}+\mathrm{PtCl}_{6} \mathrm{~K}_{2}+z \mathrm{NH}_{4} \mathrm{Cl}\right)$ obtained in the inexhaustive treatment with sal-ammoniac was, as stated, reduced with hydrogen in the wet-way, to weigh the platinum present in it. The filtrate from the platinum was evaporated to dryness, the residue made into sulphates, and thus the original potassium-apart from what had gone into the two ether-alcohol filtrates, and into the sal-ammoniac extract--recovered, as part of a mixture of alkali-sulphates. This mixture was dissolved, Finkenerised with only a small excess of platinum ( $50 \mathrm{mgs}$. of $\mathrm{Pt}$ instead of about 32 as calculated), and no "recrystallisation" effected. The washed chloroplatinate plus sulphate was treated exhaustively with sal-ammoniac $(25 \cdot 3$ grms. solution); the sal-ammoniac liquors being put aside. The chloroplatinate $\left(+x \mathrm{NH}_{4} \mathrm{Cl}\right)$ was dissolved in water, and the platinum reduced out; it weighed $24.2 \mathrm{mgs}$., equal by calculation to $18.42 \mathrm{mgs}$. of chloride of potassium.

The chloride of 'potassium was recovered from the filtrate: it weighed $18.4 \mathrm{mgs}$., including, however, $0.2 \mathrm{mg}$. of insoluble matter, hence corrected weight $=18.2 \mathrm{mgs}$. It was dissolved in water, the $\mathrm{SO}_{3}$ precipitated by nitrate of baryta (precipitate calculated as $\mathrm{Na}_{2} \mathrm{SO}_{4}=029 \mathrm{mg}$.), and the chlorine of the filtrate determined by nitrate of silver. Chloride of potassium calculated from the weight of the chloride of silver $=18 \cdot 11 \mathrm{mgs}$.

To be able to check our work, we determined the potassium in the two ether-alcohol filtrates of Experiment II., and in the two sal-ammoniac liquors, obtained in Experiments II. and II $a$. respectively.

\section{Summary of Results.}

Chloride of potassium taken, Mrgs.

$\mathrm{KCl}$ present in the chloroplatinate of II $a$.; calculated from the $\mathrm{Pt}(24.2 \mathrm{mgs}$.$) is, 18.42$

The crude $\mathrm{KCl}$ from the same, corrected by deducting $\mathrm{SO}_{3}$ as $\mathrm{Na}_{2} \mathrm{SO}_{4}$, . . . . . . .

Calculated from the chlorine of the crude chloride of potassium, .

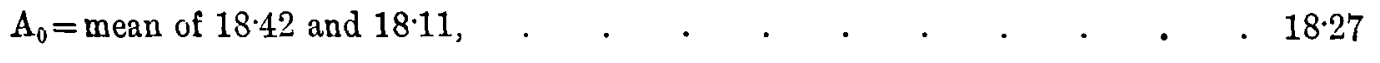


CRITICAL EXPERIMENTS FOR DETERMINATION OF POTASSIUM, ETC. 601

B. KCl obtained from first ether-alcohol filtrates of Experiment II., . . . . 073

$\mathrm{B}^{\prime}$. KCl obtained from second ether-alcohol filtrates of Experiment II., . . 0.58

C. $\mathrm{KCl}$ from the two sal-ammoniac liquors," worked conjointly:-

Calculated from $\mathrm{PtCl}_{6} \mathrm{~K}_{2}, \quad$. 4.81 , mean $=5.03$, deducting 0.23 present in " Pt, . . 5.21\} sal-ammoniac, . . . . . 480

X. Ether-alcohol filtrates of Experiment II $x$. were not worked up ; they contained, let us say, the mean of $\mathrm{B}$ and $\mathrm{B}^{\prime}, \quad . \quad . \quad . \quad . \quad . \quad .=0.65$

Total chloride of potassium found $=A_{0}+B+B^{\prime}+C+X$, . . . . . $=25.03$ Excess of $\mathrm{KCl}$ found, . $. \quad . \quad . \quad . \quad . \quad . \quad=0.88$

The relative exactitude in Experiments II. and II $a$., it is true, is not very high; but the absolute precision of the results is high, considering that the substance analysed was, virtually, a mixture of the two chlorides, $\mathrm{NaCl}$ and $\mathrm{KCl}$, which contained only 0.2766 per cent. of the latter. Hence the Finkener process, whatever it might be otherwise, is invaluable as a means for the determination of small quantities of potassium which escaped the meshes of other analytical methods, and in this sense, amongst others, we have used it largely.

\section{Experiments on synthetically prepared Mixtures containing relatively large quantities of Potassium.}

We could not detail all our experiments of this kind without filling a great many pages. We prefer to give the conclusions which we drew from a considerable experience concerning the Finkener process, and then pass on to reporting, mainly, on our final series, which was carried out by what we at the end came to recognise as the best form of the process for general purposes. The conclusions referred to are these,-

1. In the analysis of a mixture of chlorides and sulphates of the bases $\mathrm{K}_{2} \mathrm{O}, \mathrm{Na}_{2} \mathrm{O}, \mathrm{MgO}$ for $\mathrm{K}_{2} \mathrm{O}$, it is not necessary to begin by converting the bases into normal sulphates; $*$ it suffices to add a sufficient quantity of sulphuric acid, equivalent, by calculation, to the chlorine present. In the test analyses to be reported on, we added measured volumes of standard sulphuric acid calculated from our knowledge of the weight of chlorine present. In actual practice, a preliminary determination of the latter by, say, МонR's method, would give the necessary guidance.

2. In the treatment of the residue (of chloroplatinate of potassium plus sulphates) it is expedient to add, first a sufficiency of absolute alcohol, say 10 c.c., to allow to stand for some time, and then to add the necessary (5 c.c. of) ether, and allow to stand longer, but under a small bell-jar on a glass plate. For a long time it was our rule to let the alcohol act for half an hour, and the alcohol and ether for other two hours; but we subsequently found that five

* Finkener does not say it is ; but for a time it was our method. 
minutes for the alcohol and then twenty-five minutes for the alcohol and ether suffice. In two test analyses (of a " $9 \overline{5}$ per cent." salt, ride infra) we used this shorter mode, and found that the errors in the chloride of potassium amounted to only +0.09 and $+0.94 \mathrm{mgr}$., for about $753 \mathrm{mgs}$. to be determined. The mixture is filtered, and the precipitate washed with ether-alcohol. To prepare it for "recrystallisation," it is washed finally with plain ether, and allowed to dry.

3. The "recrystallisation" of the mixture $\left(\mathrm{PtCl}_{6} \mathrm{~K}_{2}+x \mathrm{R}_{2} \mathrm{SO}_{4}\right)$ obtained in the Finkener process is necessary, in general, for the removal of chloroplatinates, but addition of sulphuric acid in this subsidiary operation does no good.

4. The alcohol and the ether must be absolutely free of ammonia; we always distilled them with a little phosphoric acid before use.

5. With the generality of substances, the sal-ammoniac form of the process offers no advantages over the straight-forward determination of the platinum in the "recrystallised" precipitate (of sulphates and $\mathrm{PtCl}_{6} \mathrm{~K}_{2}$ ).

6. The determination of the platinum is best effected by reduction with hydrogen in the wet way.

In our final series, the FINKENER and the TATLOCK processes were worked side by side of one another, in this sense, that for every mixture analysed by means of one of the processes, a substantially identical mixture was analysed by the other. Yet we prefer, meanwhile, to detail our test experiments on the Finkener process first, and by themselves.

For the preparation of the mixtures to be analysed, we used the following materials:-

(1) Standard solutions of clloride of potassium made from perchlorate. The solutions were standardised synthetically by weight, and, immediately after their preparation, quantities containing the desired amounts of salt were weighed out into so many bottles, which were marked, and kept ready for use.

(2) Chloride of sodium solution, prepared from potassium-free salt, and standardised volumetrically. 1 c.c. contained $8.194 \mathrm{mgs}$. of dry salt.

(3) A sulphate of magnesia solution, prepared from pure (alkali-free) magnesia $(\mathrm{MgO})^{*}$ by solution in a very slight excess of standard sulphuric acid, and diluting to a definite volume. 1 c.c. contained $1.539 \mathrm{mgs}$. of $\mathrm{MgO}$.

(4) $A$ standard solution of sulphuric acid, made from distilled acid. 1 c.c. $=$ $47.87 \mathrm{mgs.} \mathrm{H}_{2} \mathrm{SO}_{4}$.

For the preparation of a mixture for analysis, one of the portions of chloride of potassium solution (see (1)), was mixed with measured volumes of solutions (2) and (3), and in general (4). The resulting solution was then Finkenerised * For mode of preparation, see "Challenger Memoir," p. 16. 
CRITICAL EXPERIMENTS FOR DETERMINATION OF POTASSIUM, ETC. 603

with a measured volume of a standard solution of chloroplatinic acid prepared from chemically pure metal by (in most cases) the chlorine process (see page 564).

Our final experiments were made in sets of, in general, four analyses of the same kind of mixture. Before passing to these sets, we will shortly report on a single experiment, made with the view of seeing how the Fin KENER process works with relatively pure potassium salts.

\section{Analysis of a Mixture of Sodium and Potassium Chlorides, containing $98 \cdot 4$ per cent of $\mathrm{KCl}$.}

The solution analysed contained $7.1 \mathrm{mgs}$. of $\mathrm{Na}_{2} \mathrm{SO}_{4},=5.9 \mathrm{mgs}$. of $\mathrm{NaCl}$, and exactly 0.3701 grms. of $\mathrm{KCl}$. It was mixed with sulphuric acid equal to $49 \mathrm{mgs}$. of $\mathrm{H}_{2} \mathrm{SO}_{4}$ (to give the sodium a better chance of separating out as sulphate), and then Finkenerised with 11 c.c. of a platinum solution, of which 10 c.c. would have sufficed by calculation.* Salt-mixture $\left(x \mathrm{R}_{2} \mathrm{SO}_{4}+\mathrm{PtCl}_{6} \mathrm{~K}_{2}\right)$ " recrystallised." Platinum obtained from the final chloroplatinate (by reduction in the wet way) $=0.4855 \mathrm{grm} .=0.3696 \mathrm{grm}$. of $\mathrm{KCl}$ : error, $=-0.5 \mathrm{mg}$.

The second ether-alcohol filtrate was worked up for potassium. It amounted to $0.3 \mathrm{mg}$. of $\mathrm{KCl}$. Assuming the first ether alcohol filtrate (which was not analysed) to have contained the same quantity, we have-

Total $\mathrm{KCl}$ recovered, $=369 \cdot 6+0 \cdot 6=0.3702$, instead of $0.3701 \mathrm{grm}$. The weight of platinum eliminated by "recrystallisation" was $3 \cdot 1$ mgs. Hence, if this operation had been omitted, we should have had $0.4855+0.0031=0.4886 \mathrm{grm}$. of platinum, equal to 0.3719 of $\mathrm{KCl} ;$ i.e., a positive error $=1.8 \mathrm{mgs}$.

We now pass to the series of trials referred to. $\dagger$

\section{Set of Experiments.}

Subject :-a " 95 per cent." salt. $\$$ Chloride of potassium operated upon in each analysis, about $0.75 \mathrm{grm}$. Sulphuric acid required to replace every $\mathrm{Cl}$ of the mixture by $\frac{1}{2} \mathrm{SO}_{4},=10 \cdot 9$ c.c. ; actually added, $=12 \cdot 0$ c.c. The whole was evaporated to dryness, and the residue ignited to expel the chlorine. Platinum solution required by calculation, $=20$ c.c., we added 21.5 c.c., i.e., a very small excess, which, as we now know, was a mistake. The mixture of $\mathrm{PtCl}_{6} \mathrm{~K}_{2}$ and

* See last line of this page.

tThe designations of the following sets of experiments do not in general indicate the order in which they were carried out.

$\ddagger$ Meaning a mixture containing 95 parts of chloride of potassium in 100 of total anhydrous salts. 
sulphates obtained was "recrystallised," the crystals obtained dissolved, the platinum reduced out by hydrogen and weighed.*

\begin{tabular}{|c|c|c|c|c|c|}
\hline \multicolumn{6}{|c|}{ Results in Granmes. } \\
\hline $\begin{array}{l}\text { Experiment. } \\
\text { I. KCl taken, }\end{array}$ & $\begin{array}{c}1 . \\
\cdot 75750\end{array}$ & $\begin{array}{c}2 . \\
.75984\end{array}$ & $\begin{array}{c}3 . \\
.75791\end{array}$ & $\begin{array}{c}4 . \\
\cdot 76042\end{array}$ & $\begin{array}{c}5 . \\
.75982\end{array}$ \\
\hline II. Platinum obtained, & .9959 & .9952 & .9917 & .9945 & .9991 \\
\hline III. $\mathrm{KCl} \mathrm{if}=0.76117 \times$ II., & .75805 & $\cdot 75752$ & 75485 & 75698 & 76048 \\
\hline Error, i.e., III.-I., & +.55 & $-2 \cdot 32$ & -3.06 & $-3 \cdot 44$ & $-0.66 \mathrm{mgs}$ \\
\hline
\end{tabular}

The first alcohol and ether washings from all the five analyses were worked up for potassium by FinkeneR's method, sal-ammoniac form. Ultimately the $\mathrm{KCl}$ was determined in Fresenius's way, as $\mathrm{PtCl}_{6} \mathrm{~K}_{2}$. Found for the five analyses, 1.37 mgs. of $\mathrm{KCl}$.

The second alcohol and ether washings contained $39.1 \mathrm{mgs}$. of platinum, equal to $29 \cdot 76$ of chloride of potassium, or 5.95 mgs. per analysis. So much more would have been found (than quantities III.) if the recrystallisation had been omitted. But a determination of the potassium (as $\mathrm{Pt}$ ) showed that $0.26 \mathrm{mg}$. of $\mathrm{KCl}$ per analysis was present in the ether-alcohol liquor.

Viewing the five analyses as one, we have -

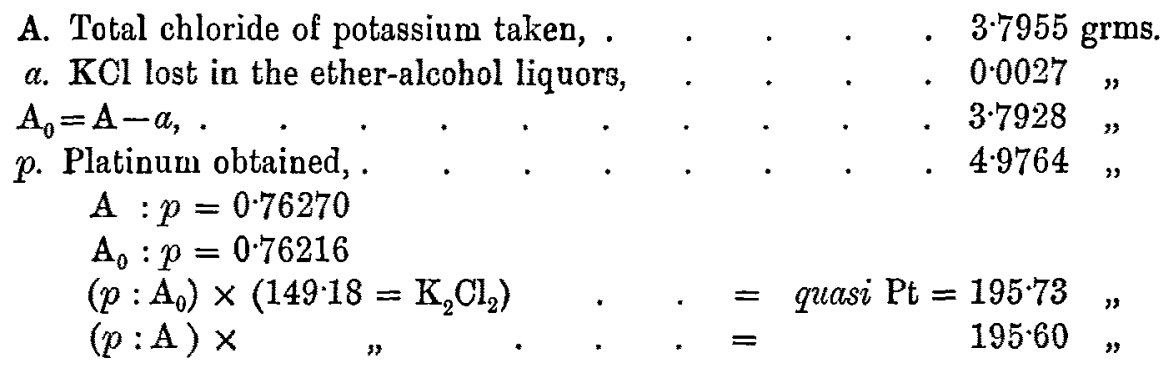

\section{Set of Experiments.}

This set was carried out before we had come to adopt the recrystallisation modus for purifying the FinkENER product.

It seemed to us at the time that the most exact method of potassium determination would be to eliminate the bulk of the potassium as chloroplatinate by precipitation from purely aqueous solutions, and to utilise the Finkener process only for the recovery of the unprecipitated remnant.

The substance worked upon was the 95 per cent. salt used in the I. Set. A known weight of chloride of potassium (amounting to about $0.76 \mathrm{grm}$.) was dissolved with the necessary impurities, and the solution next evaporated to about 5 c.c. About 15 times the calculated minimum of platinum solution was now added to produce some 35 c.c. of mixture, which was allowed to stand over night. 'The mother-liquor was then decanted off through a small filter,

* In only one case, No. (4), did the filtrate contain a trace of platinum. It was recovered by $\mathrm{H}_{2} \mathrm{~S}$, the $\mathrm{Pt} \mathrm{S}_{2}$ made into $\mathrm{Pt}$, and its weight $(0.4 \mathrm{mg}$.) added on. 
the precipitate washed with small instalments of water until pure by calculation (18 c.c. of water were used in all), then with dilute, and finally with absolute alcohol. The precipitate was dried at $110^{\circ}$ and weighed. It was then dissolved in water, the platinum reduced out by hydrogen, and weighed likewise.

The alcoholic washings were evaporated to dryness, the aqueous liquors added, and after addition of enough of standard sulphuric acid for replacing the $\mathrm{Cl}$ of the chlorides present by $\frac{1}{2} \mathrm{SO}_{4}$, the whole evaporated, Finkenerised, and the sulphates removed by sal-ammoniac solution. The resulting chloroplatinate was dissolved in hot water, reduced by hydrogen, the platinum filtered off, and the filtrate next evaporated to dryness, and the residue ignited, to drive off the ammonia-salt. In the ignited salt, the potassium was determined by means of the FRESENIUS' form of the chloroplatinate process. In some cases the chloroplatinate obtained was reduced in hydrogen (wet way), and the platinum weighed.

The factors used were -

For reducing $\mathrm{PtCl}_{6} \mathrm{~K}_{2}$ to $\mathrm{K}_{2} \mathrm{Cl}_{2}$,

In Experiments II. to V. chloroplatinic acid made by the chlorine process was used; in Experiment I. a reagent made with aqua regia.

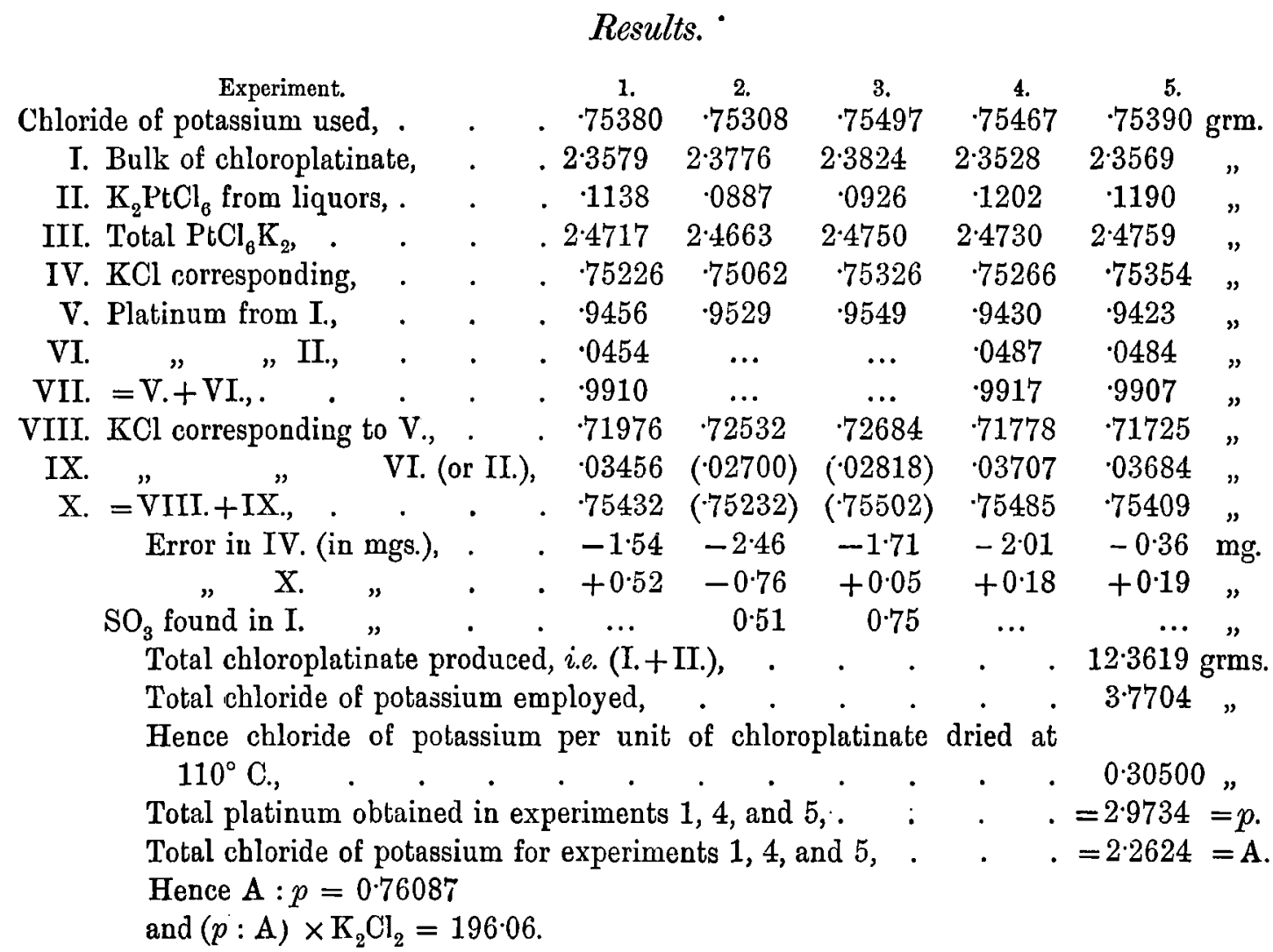




\section{Set of Experiments.}

Subject, the same " 95 per cent." salt as was used in Sets I. and II. Method the same as that used in Set I.; except that the salts were made into sulphates only virtually, by addition of the calculated amount of standard sulphuric acid. Platinum added on Finkenerising, $=25$ c.c. or $1 \cdot 25$ times the calculated minimum.

Product $\left(\mathrm{PtCl}_{6} \mathrm{~K}_{2}\right.$ and $\left.x \mathrm{R}_{2} \mathrm{SO}_{4}\right)$ "recrystallised."

\section{Results.}

\begin{tabular}{|c|c|c|c|c|}
\hline $\begin{array}{l}\text { Exporiment. } \\
\text { loride of potassium taken, }\end{array}$ & $\begin{array}{c}1 . \\
\cdot 75330\end{array}$ & $\begin{array}{l}2 . \\
\cdot 75253\end{array}$ & $\begin{array}{c}3 . \\
\cdot 75327\end{array}$ & $\begin{array}{c}4 . \\
75426 \mathrm{grm}\end{array}$ \\
\hline II. Platinum obtained, & 9900 & 9880 & .9902 & $9907^{\circ}$, \\
\hline II. $\mathrm{KCl}$ found if $=\cdot 7$ & $\cdot 75356$ & $\cdot 75204$ & $\cdot 75371$ & 75409 \\
\hline
\end{tabular}

The ether-alcohol liquors not analysed.

Uniting the four analyses into one, we have-

Chloride of potassium taken, . . . . . $=\mathrm{A}=3.0134 \mathrm{grms}$.

Platinum obtained, . . . . . . . $=p=3.9589$,

Hence $\mathrm{A}: p .=0.76116$.

$(p: \mathrm{A}) \times \mathrm{K}_{2} \mathrm{Cl}_{2}=" \mathrm{Pt} "=195 \cdot 99$.

\section{Set of Experiments.}

The solutions analysed were such as to represent very nearly a salt consisting of 82 per cent. of chloride of potassium, 15 of chloride of sodium, and 3 of sulphate of magnesia. The quantity of chloride of potassium used was about 0.656 grm. per analysis.

The solution to be analysed was mixed with the quantity of standard sulphuric acid equivalent to the chlorides present, and 1.25 times the calculated volume of platinum solution, and Finkenerised; the resulting product being " recrystallised." All else as in III.

Results.

Experiment.
I. Chloride of potassium taken,

II. Platinum obtained,

III. $\mathrm{KCl}$, corresponding $=$ II. $\times 0.76117,$.

\begin{tabular}{llll}
1. & \multicolumn{1}{c}{${ }^{2}$} & \multicolumn{1}{c}{4.} \\
.65390 & -65294 & 65032 & .65046 grm. \\
.8597 & .8553 & .8543 & $.8547 \quad "$ \\
.65438 & .65103 & 65027 & .65057 \\
+0.48 & -1.91 & -0.05 & $+0.11 \mathrm{mg}$.
\end{tabular}


CRITICAL EXPERIMENTS FOR DETERMINATION OF POTASSIUM, ETC. 607

Uniting the four analyses into one, we have-

Total chloride of potassium used, . . . . $=26076 \mathrm{grms} .=\mathrm{A}$.

" platinum obtained, . . . . . . . $=3 \cdot 4240 "=p$.

Hence A : $p=0.76157$

and $(p: \mathrm{A}) \times \mathrm{K}_{2} \mathrm{Cl}_{2}=195.89$.

\section{Set of Experiments.}

Salt analysed consisted of 33.3 per cent. of chloride of potassium, 33.3 of chloride of sodium, and 33.3 of sulphate of magnesium.

Method exactly as in IV. Set.

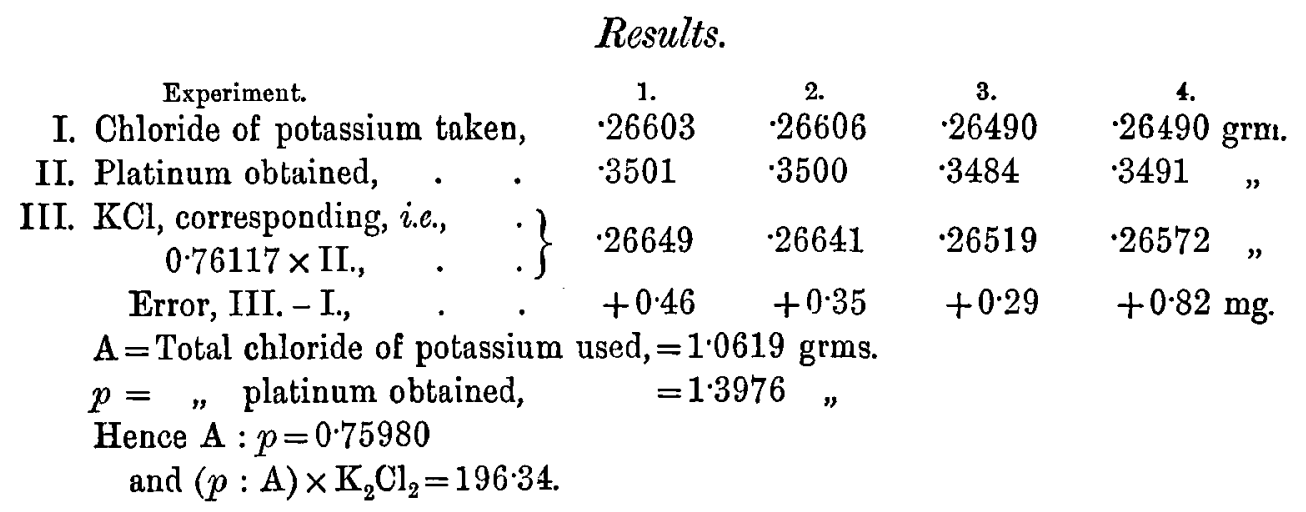

Tatlock's Method.

This method was invented expressly for the assaying of commercial potashsalts, i.e., of salt mixtures similar in constitution to those which we employed for our test-analyses by Finkener's method. Mr TatLock's method, according to his own description,* is as follows:-Assuming the substance to be analysed to have been converted into a standard solution, a quantity equal to ("10 grains" =) 0.6 to $0.7 \mathrm{grm}$. of dry salt is measured off, to be analysed as follows :-For every one gramme of salt the solution is diluted to about 40 c.c. ; it is then acidified with a few drops of hydrochloric acid, and mixed with 50 c.c. of a " 5 per cent." chloride of platinum solution, meaning a solution containऽ centgrms. of metal per c.c. The mixture is evaporated to near dryness over a water-bath, and the residue re-evaporated with addition of a little water, to more fully eliminate the free hydrochloric acid. [Observe that this large proportion of platinum is prescribed for all kinds of salts, rich or poor. Now 1 grm. each of the anhydrous salts, $\mathrm{NaCl}, \mathrm{MgCl}_{2}, \mathrm{MgSO}_{4}$, demands only 169 , $2 \cdot 08,1.65$ grms. of platinum, assuming $\mathrm{Pt}$ to be equal to 198 ; hence $\mathrm{Mr}$ TaTlock's intention apparently is to have sufficient chloroplatinic acid present for converting all the metals into chloroplatinates, and, in addition thereto, some

* As communicated by him to a Committee of the British Association, and published by them in a Report presented to the Meeting at Glasgow, in 1876. 
$0.8 \mathrm{grm}$. (we calculate from the $\mathrm{NaCl}$ number) as surplus chloroplatinic acid.] Some 5 c.c. more of the choride of platinum solution are mixed with the residue (which, in the case of $\mathrm{NaCl}$, will produce some 6 c.c. of a 17 per cent. solution); the whole is stirred well, and set aside in a cool place for at least an hour, with occasional stirring. The precipitate is then thrown on a very small filter, the basin rinsed out with about 15 drops of the platinum solution, and the precipitate on the filter washed with 16-24 drops more. The basin, and filter and contents, are then washed with the smallest possible quantity of alcohol of 95 per cent. (by weight or volume?; we used 95 per cent. by weight), and dried at $100^{\circ} \mathrm{C}$. The precipitate is transferred as far as possible to a tared capsule, and further dried until it assumes a distinct orange colour. The filter with the remnant of precipitate adhering to it is incinerated, and the residue calculated as $\mathrm{Pt}+\mathrm{K}_{2} \mathrm{Cl}_{2}$. The weight of the chloroplatinate of potassium, multiplied by $0 \cdot 3056$, gives the weight of chloride of potassium to be determined. [The factor is calculated from $\mathrm{Cl}=35 \cdot 457 ; \mathrm{K}=39 \cdot 137 ; \mathrm{Pt}=197 \cdot 19$.]

Whenever in the following we state that an analysis was executed according to "Tatlock's directions," these directions were followed closely as above given, except that we allowed ourselves to recover the small quantity of chloroplatinate sticking to the filter, by dissolving it off in hot water, and evaporating the solution to dryness in the tared crucible intended to receive the main quantity, and that we continued the drying process at $100^{\circ}$ until the weight became constant.

In the case of a substance rich in sulphates, TatLock recommends to add a quantity of pure chloride of sodium. This rule, however, is obviously based on the misapprehension that "platinum solution" is one of $\mathrm{PtCl}_{4}^{*}$ while it really is one of $\mathrm{PtCl}_{6} \mathrm{H}_{2}$. Yet the chloride of sodium may do good by substituting acid sulphate of soda for the $\mathrm{H}_{2} \mathrm{SO}_{4}$ liberated, and besides, by displacing some of the $\mathrm{HCl}$ in the surplus $\mathrm{PtCl}_{6} \mathrm{H}_{2}$.

Of the various sources of error involved in TATLOCK's method, the most obvious is the appreciable solubility of chloroplatinate of potassium in water, and aqueous liquids generally. We therefore, at an early stage of our investigation, determined the

\section{Solubility of the Chloroplatinate}

in the following reagents :-Five small Hasks were tared, each charged with 0.2 grm. of chloroplatinate of potassium, and a convenient volume of the respective

* We used to be under this erroneous impression ourselves until some three years ago, when we analysed a carefully prepared platinum solution (which had been specially freed from extra hydrochloric acid) for chlorine and platinum. It contained very nearly $6 \times \mathrm{Cl}$ for $1 \times \mathrm{Pt}$, which, by the way, is in accordance with an old analysis of "chloride of platinum," quoted in GmeLins's handbook as having been made by VAUQUELIN. 
solvent, the flasks stopped up, and allowed to stand with occasional agitation. Whenever the precipitate threatened to dissolve completely, an additional weighed instalment of chloroplatinate was added. After six days the contents of the flasks were weighed, filtered, and the filtrates analysed for the dissolved chloroplatinate. The temperature during those six days varied from about $13^{\circ}$ in the mornings, to about $16^{\circ} .5$ in the evenings. The results were as follows :-

100 parts by weight of solvent dissolve $q$ parts of $\mathrm{PtCl}_{6} \mathrm{~K}_{2}$.
A. Water, Solvent.
B. Hydrochloric acid of 5 per cent., . . . . . . . . . . 0.662
C. "5 per cent." chluride of platinum solution, . . . . . . . 0.233
D. Solution of chloride of platinum, containing $0.05 \mathrm{grm}$. of added (real)
$\mathrm{HCl}$, and $0.05 \mathrm{grm}$. of platinum, per c.c., . . . . . 0.168
E. Sulphuric acid, containing $\frac{1}{2} \mathrm{SO}_{3}=40$ grms. per litre, . . . . 0.900

Methods of Analysis: A. and B.-Evaporation to dryness, and weighing of residue, dried at $150^{\circ} \mathrm{C}$., as $\mathrm{PtCl}_{6} \mathrm{~K}_{2}$.

C. and D.-Evaporation to dryness on a water-bath, treatment with absolute alcohol, and weighing of the washed precipitate after drying at $150^{\circ}$ as $\mathrm{PtCl}_{6} \mathrm{~K}_{2}$.

E.-The liquid almost neutralised with pure (potassium-free) caustic soda, the platinum reduced out by hydrogen, and weighed.

We regret now not to have determined the action of stronger solutions of chloroplatinic acid, because Mr TATLOCK virtually begins by washing his chloroplatinate with a " 17 per cent." solution of the reagent.

In now passing to our analyses, we begin with a series in which we deliberately departed from certain of Mr TATLOck's rules, in order to bring the errors into greater prominence, and also on the chance of being able to rectify these by suitable modifications of the process. Let us at once confess that our success in the latter direction amounted to very little, if anything.

\section{Preliminary Trials.}

Experiment I.-The solution analysed contained exactly $0.3471 \mathrm{grm}$. of chloride of potassium, and about $100 \mathrm{mgs}$. $\mathrm{Na}_{2} \mathrm{SO}_{4}$, and $150 \mathrm{mgs}$. of $\mathrm{MgSO}_{4}$; it consequently represented a salt of " 58 per cent." Evaporated down with platinum solution equal to $515 \mathrm{mgs}$. of platinum, or $50 \mathrm{mgs}$. more than demanded by the potassium. Residual magma washed five times, each time with 0.5 c.c. of water, then exhaustively with absolute alcohol. The chloroplatinate dried at $150^{\circ}$, and weighed; then dissolved in water, the platinum reduced out, and weighed likewise. 
The aqueous washings were evaporated with 1 c.c. of normal sulphuric acid (49 mgs. of $\mathrm{H}_{2} \mathrm{SO}_{4}$ ) to a magma and Finkenerised; no recrystallisation; sal-ammoniac form applied. Resulting chloroplatinate dissolved, platinum reduced out and weighed.

Alcoholic Washings.-After removal of the alcohol by distillation, the platinum was removed by hydrogen, the filtrate evaporated to dryness, residual salts made into neutral sulphates and Finkenerised; sal-ammoniac process.

The results were as follows (mgs.) :-

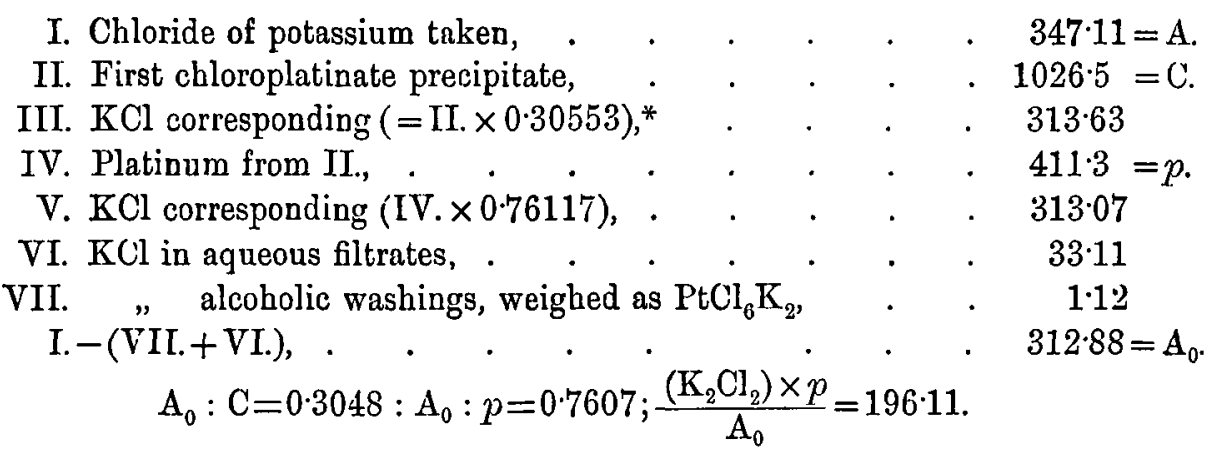

Conclusion.-It is quite possible, by operating as described, i.e., without wasting so much platinum as TATLOCK does, to obtain a chloroplatinate fit for the balance; but the chloroplatinate includes only about 90 per cent. of the chloride of potassium.

Experiment II.-The solution analysed represented 1.04 grms. of a " 67 per cent." salt, including 0.24 grm. of $\mathrm{Na}_{2} \mathrm{SO}_{4}, 0.20$ of $\mathrm{NaCl}$, and 0.10 of $\mathrm{MgCl}_{2}$. Platinum used, $2 \cdot 6$ grms., or $2 \cdot 49$ per grm. of salt analysed. Tatlock's directions followed, except that the washing with 5 per cent. platinum solution was continued until the last runnings contained only a trace of $\mathrm{SO}_{4} \mathrm{R}_{2}$. Washing completed with 95 per cent. alcohol. Precipitate weighed, after drying at $100^{\circ}$, and after further drying at $150^{\circ} . A=0.7018$ grm. Chloroplatinate obtained, dried at $100^{\circ}=\mathrm{C}^{\prime}=2 \cdot 2745$ : same dried at $150^{\circ}=\mathrm{C}^{\prime \prime}=2 \cdot 2737$. Platinum from $\mathrm{C}$, by wet-way reduction, $=0.9132 \mathrm{grm} .=p$. Potassium in filtrates collected by Finkener's (sal-ammoniac) process, and weighed as $\mathrm{PtCl}_{6} \mathrm{~K}_{2} .-\mathrm{KCl}$ thus found $=0.00819$.

$\mathrm{C}^{\prime} \times 0.3043 \mathrm{j}^{*}=0.69224 ; p \times 0.76117=0.69510$.

Mean $=0.69367$; loss $=0.00813,=1 \cdot 16$ per cent. of the $\mathrm{KCl}$ taken. $\mathrm{KCl}$ in precipitate by synthesis $=0.69361=A_{0}$.

$\mathrm{A}_{0}: \mathrm{C}^{\prime}=0.30500 ; \mathrm{A}_{0}: p=0.75954$.

$\frac{p \times \mathrm{K}_{,} \mathrm{Cl}_{?}}{\mathrm{~A}_{0}}=196.41$.

Conclusion.-It will not do to "improve" upon Tatlock's method by washing with platinum solution, until the $\mathrm{SO}_{3}$ is proved to be away.

* These factors were calculated from results for " $M$ " in the first series of potassium experiments. We did not consider it necessary to recalculate the analyses with our present factors. 


\section{Further Trials.}

These were carried out with solutions representing 95 per cent., 82 per cent., 33.3 per cent. salts, which were prepared from the same materials as those used for the corresponding trials with Finkener's method. In the case of each kind of salt, indeed, the two methods were worked side by side of each other, so as to give no advantage to either.

General Method.-A solution representing 0.648 grm. of a " 95 per cent." salt mixed with a few drops of hydrochloric acid, and 324 c.c. of 5 per cent. chloride of platinum; mixture evaporated to a magma, mixed with 2 to 3 c.c. of water, and evaporated again. After cooling, 3.25 c.c. of platinum solution added, and allowed to stand for an hour. So far, all the four analyses conducted in the same way.

In (1) and (2).-Washing with chloroplatinic acid continued until the impurities by calculation were reduced to about $0.06 \mathrm{mg}$. and the $\mathrm{SO}_{3}$ to 0.012 (but a direct test with $\mathrm{BaCl}_{2}$ showed that there must have been more). The washing then completed with 95 per cent. alcohol.

In (3).- After decanting off the mother-liquor, the precipitate was washed once, with 1 c.c. of platinum solution. The precipitate was then dissolved in hot water, and the solution, after addition of 2 c.c. of platinum solution, re-evaporated as far as possible on a water-bath. 2 c.c. of water were then added, and the whole allowed to stand for an hour. The precipitate was then filtered off, washed with two successive cubic centimetres of platinum solution (when as a matter of calculation, the impurities should have been reduced to $0.25 \mathrm{mg}$.; the $\mathrm{SO}_{3}$ to $0.06 \mathrm{mg}$, yet a drop tested with $\mathrm{BaCl}_{2}$ gave a precipitate), and lastly with 95 per cent. alcohol.

In (4), TATlock's directions were strictly obeyed.

Chloroplatinates dried at $100^{\circ}$ to $105^{\circ}$, weighed, and reduced with hydrogen, (wet way), to determine their platinum.

The results are stated in the following table (in grammes) :-

\begin{tabular}{|c|c|c|c|c|}
\hline Experiment, & 1. & 2. & 3. & 4. \\
\hline I. Chloride of potassium taken, . & $\cdot 61075$ & 61033 & 60992 & 60917 \\
\hline II. Chloroplatinate obtained, & 1.9721 & 1.9696 & 1.9755 & $1 \cdot 9860$ \\
\hline III. Platinum from "II.", . & 7927 & 7918 & 7947 & $\cdot 7964$ \\
\hline IV. II. × 0.30435, & 60021 & .59945 & $\cdot 60124$ & 60444 \\
\hline Error = IV. minus I.; mgs., & $-10 \cdot 54$ & $-10 \cdot 88$ & $-8 \cdot 68$ & $-4 \cdot 73$ \\
\hline V. III. ×0.76117, . . & 60338 & $\cdot 60269$ & 60490 & $\cdot 60620$ \\
\hline Error $=$ V. minus I.; mgs., & $-7 \cdot 37$ & $-7 \cdot 64$ & $-5 \cdot 02$ & $-2 \cdot 97$ \\
\hline
\end{tabular}

Filtrates-from (1), (2), and (3), ${ }^{*}$ freed from alcohol by distillation : their potassium recovered by FINKENER's (sal-ammoniac) process, and determined

* Those of (4) were lost by a disaster in the laboratory. 
as $\mathrm{PtCl}_{6} \mathrm{~K}_{2}$. The $\mathrm{KCl}$ recovered amounted to $22 \cdot \overline{0} \overline{\mathrm{j}}$ mgs. or $7 . \overline{5} 2 \mathrm{mgs}$. per analysis.

Uniting analyses (1), (2), and (3) into one, using the same symbols as before, we have-

$$
\mathrm{A}_{0}: \mathrm{C}=\cdot 30563 ; \mathrm{A}_{0}: p=76011 ;\left(p: \mathrm{A}_{0}\right) \mathrm{K}_{2} \mathrm{Cl}_{2}=196 \cdot 26 \text {. }
$$

For experiment (4) : by our analysis A : $\mathrm{C}=0.30673$.

The Committee's (TatLock's) factor is 0.30560 .

\section{Final Experiments.}

These were all carried out strictly according to TATIOCK's directions.

\section{Series.}

In it, a 95 per cent. salt, virtually the same as that used for Set I. of the Frnkener analyses, was used. Chloride of potassium taken per analysis, 6104 to $6124 \mathrm{grm}$. The chloroplatinates dried at $100^{\circ}$, and their weights multiplied by the Committee's factor, 0.30560 , to find the chloride of potassium. The errors were, in

$$
\begin{aligned}
& \text { Analysis, (1) } \\
& -1.60 \quad-1.60
\end{aligned}
$$

The platinum of each precipitate was determined as usual; calculating from the weight of the platinum, by multiplying with 0.76117 , the errors were-

$$
\begin{array}{cccc}
(1) & (2) & (3) & (4) \\
-2 \cdot 66 & -3 \cdot 43 & -0.30 & -1 \cdot 58 \mathrm{mgs.}
\end{array}
$$

The volume of the platinic washings $=46$ c.c. ; that of the alcoholic, about 19 c.c. per analysis.

Total $\mathrm{KCl}$ recovered from united washings (by FrNkENER's sal-ammoniac process), determined as $\mathrm{PtCl}_{6} \mathrm{~K}_{2}=11.11$ mgs., or $2.78 \mathrm{mgs}$. per analysis.

Uniting the four analyses into one, we have-

$$
A=2 \cdot 44447 \text { grms. } \quad A_{0}=2 \cdot 43336 \quad C=7 \cdot 9880
$$

Hence $\mathrm{KCl}$ by Committee's factor, $=244112$, = "T."

$\mathrm{T}-\mathrm{A}_{0}=+7 \cdot 76 \mathrm{mgs} . ; \mathrm{T}-\mathrm{A}=-3.35 \mathrm{mgs}$, showing that the smallness of the latter difference is owing to impurities in the chloroplatinate.

Calculating constants from the slumped analysis, we have-

$$
\begin{aligned}
& \mathrm{A}: \mathrm{C}=0.30602(\text { Committee's factor }=0.30560) . \\
& \Lambda_{0}: \mathrm{C}=0.30463 \quad \mathrm{~A}_{0}: p=0.76019 \quad\left(p: \mathrm{A}_{0}\right) \mathrm{K}_{2} \mathrm{Cl}_{2}=196 \cdot 24
\end{aligned}
$$

Let us note down before passing on, that the $\mathrm{KCl}$ found in the filtrates amounted to $11 \cdot 11 \div 18 \cdot 6,=0.597 \mathrm{mg}$. per c.c. of aqueous platinic washings. 
CRITICAL EXPERIMENTS FOR DETERMINATION OF POTASSIUM, ETC. 613

II. Series : with 82 per cent. Salt.

Comprising again 4 analyses, each made with about $0.52 \mathrm{grm}$. of chloride of potassium.

Taking " $\mathrm{T}$ " as symbol for the weight of chloride of potassium found in an analysis, from the chloroplatinate by the Committee's factor, and $A$ as designating the corresponding weight of chloride of potassium taken, we had :-

\begin{tabular}{|c|c|c|c|c|}
\hline Anal & (1) & (2) & (3) & (4) \\
\hline $\mathrm{T}-\mathrm{A}$ in mgs., & $-1 \cdot 49$ & -0.90 & $-1 \cdot 21$ & $-1 \cdot 22$ \\
\hline Volume of platinic filtrates, & $4 \cdot 8$ & $4 \cdot 8$ & $4 \cdot 8$ & 5.0 c.c \\
\hline
\end{tabular}

These, united with the alcoholic washings, contained in all $9.71 \mathrm{mgs}$. of $\mathrm{KCl}$, or 2.43 per analysis, or $0.500 \mathrm{mg}$. per c.c. of aqueous platinic filtrates.

Uniting the four analyses into ane, we had-

$$
\begin{aligned}
& \mathrm{A}=2 \cdot 10646 ; \mathrm{A}_{0}=2 \cdot 09675 ; \mathrm{C}=6.8771 ; \\
& \mathrm{T}=2 \cdot 10164 ; \mathrm{T}-\mathrm{A}=-4 \cdot 82 \mathrm{mgs.} ; \mathrm{T}-\mathrm{A}_{0}=+4.89 \mathrm{mgs} . \\
& \mathrm{A}: \mathrm{C}=0.30630 ; \mathrm{A}_{0}: \mathrm{C}=0.30489 . \\
& \text { From }(1),(3) \text { and }(4) . \quad \text { Total platinum }=p=2.0689 ; \text { (platinum from (2) lost). } \\
& \quad \mathrm{A}_{0}=1.57274 . \\
& \mathrm{A}_{0}: p=0.76018 ;\left(p: \mathrm{A}_{0}\right) \mathrm{K}_{2} \mathrm{Cl}_{2}=196 \cdot 24 .
\end{aligned}
$$

III. Series : 33.3 per cent. Salt.

Four analyses, each with about 0.215 grm. of chloride of potassium.

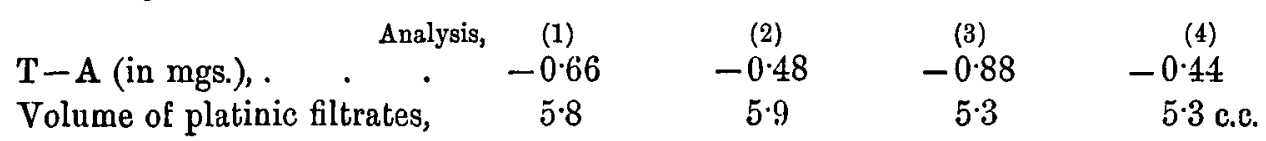

From all the filtrates, including alcoholic, $\mathrm{KCl}$ recovered as usual, and found, $=9 \cdot 10 \mathrm{mgs}$, or $2 \cdot 28 \mathrm{mgs}$. per analysis, or $0.408 \mathrm{mg}$. per c.c. of aqueous platinic filtrates.

Uniting the four analyses into one, we had-

$$
\begin{aligned}
& \mathrm{A}=0.86132 ; \mathrm{A}_{0}=0.85222 ; \mathrm{C}=2.8104 ; p=1.1259 ; \\
& \mathrm{T}=0.85886 ; \mathrm{T}-\mathrm{A}=-2.46 \mathrm{mgs} ; \mathrm{T}-\mathrm{A}_{0}=+6.64 \mathrm{mgs} . \\
& \mathrm{A}: \mathrm{C}=0.30648 ; \mathrm{A}_{0}: \mathrm{C}=0.30324 ; \mathrm{A}_{0}: p=0.75692 ;\left(p: \mathrm{A}_{0}\right) \mathrm{K}_{2} \mathrm{Cl}_{2}=197.09 .
\end{aligned}
$$

The precipitate obviously was impure, and included foreign chloroplatinates.

Before passing on, let us summarise the principal result of our test analyses by the two methods.

\section{A. Finkener's.}

Each set of analyses united into one. Column I. refers to the respective page of this memoir; Column II. specifies the set of analyses referred to; Column III. the percentage of $\mathrm{KCl}$ in the salt analysed. 


\begin{tabular}{|c|c|c|c|c|c|}
\hline Page. & Set. & $\begin{array}{l}\text { Per cents. of } \\
\mathrm{KCl} \text { in Salt. }\end{array}$ & $\Delta: p$ & $\left(p:\right.$ A) $\mathrm{K}_{2} \mathrm{Cl}_{2}$ & $\left(p: A_{0}\right) \mathrm{K}_{2} \mathrm{Cl}_{2}=195 \cdot 73$ (Set I.) \\
\hline 604 & I. & 95 & $(\cdot 76270)$ & $195 \cdot 60$ & \multirow{7}{*}{$\begin{array}{c}\text { Mean }\left(p: A_{0}\right) \mathrm{K}_{2} \mathrm{Cl}_{2} \text { may } \\
\text { perhaps be put down at } \\
195 \cdot 98+0 \cdot 13=196 \cdot 11 .\end{array}$} \\
\hline 605 & II. & 95 & $(\cdot 76087)$ & 196.06 & \\
\hline 606 & III. & 95 & 76116 & $195 \cdot 99$ & \\
\hline 606 & IV. & 82 & $\cdot 76157$ & $195 \cdot 89$ & \\
\hline \multirow[t]{3}{*}{607} & V. & $33: 3$ & $\cdot 75980$ & $196 \cdot 34$ & \\
\hline & \multicolumn{2}{|c|}{ Means, } & $\cdot 76084$ & $195 \cdot 98$ & \\
\hline & Cal & ulated from &., IV., \& V & All. & \\
\hline
\end{tabular}

Set I. Salts made into normal sulphates; platinum used $=1.075$ times the calculated minimum.

Set II. A combination of TATLOCK's and Finkener's methods.

Set III. Only sulphuric acid added; no evaporation : platinum used, =1.25 times the calculated minimum.

Only sets III., IV., and V. correspond to our present method: hence the mean factor $\mathrm{A}: p$ was calculated from only these 3 sets.

We have recalculated the 12 analyses of sets III., IV., and V., with the new factor $0 \cdot 76084$, and found the "errors" of the recalculated numbers.

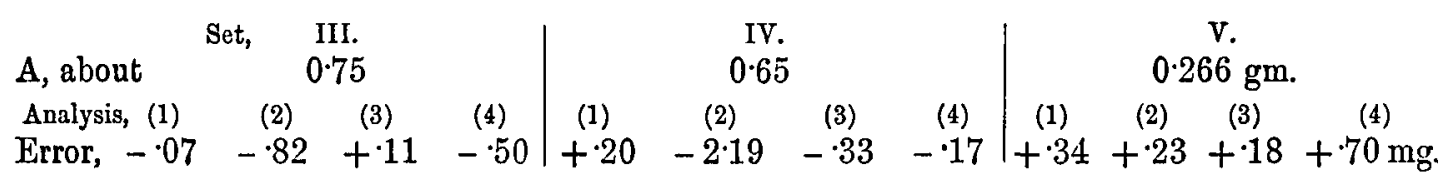

Mean error $= \pm 0.49 \mathrm{mg}$, or, excluding No. 2 in IV., it is \pm 0.33 .

\section{B. Tatlock's.}

In the following table, the first column refers to the respective page of this memoir ; the third gives the percentage of $\mathrm{KCl}$ in the set of salts analysed; the figures in the second are reference marks.

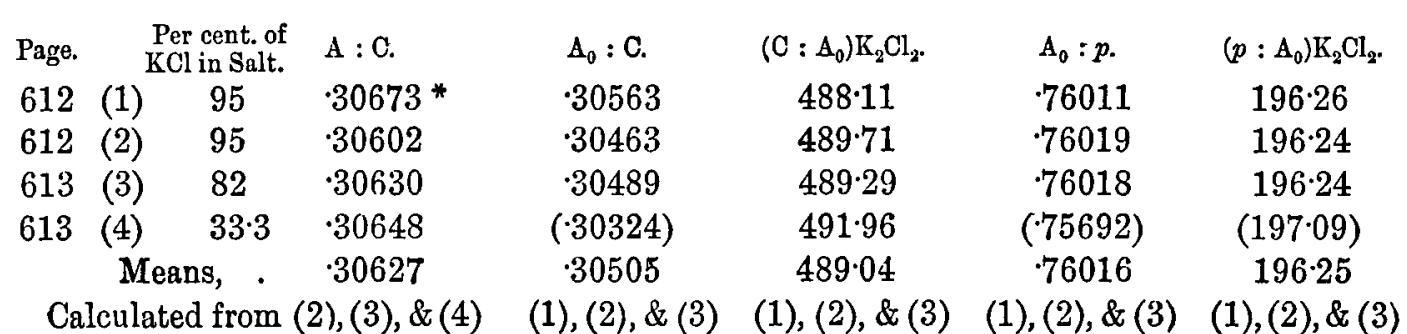

Recalculating the 12 analyses we made (by TArLock's exact method) with the factor 0.30627 , we arrive at the following errors for the individual results :-

* From the one analysis of this set in which Tatlock's directions were strictly obeyed. 
CRITICAL EXPERIMENTS FOR DETERMINATION OF POTASSIOM, ETC. 615

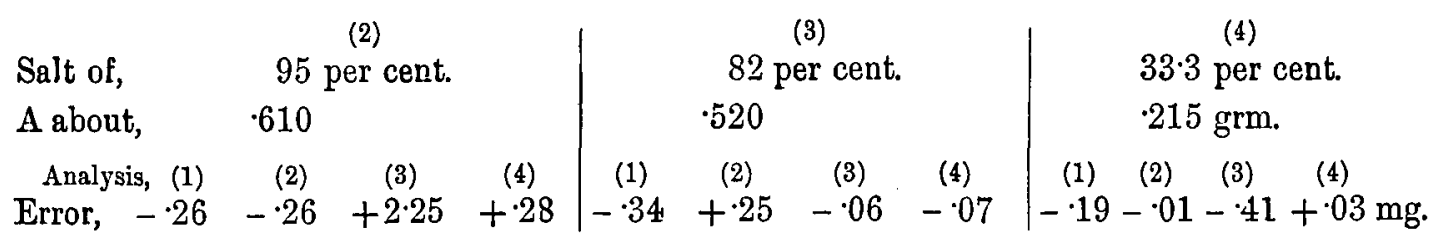

Mean error, $= \pm 0.37$, or excluding No (3) under (2), $= \pm 0.20 \mathrm{mg}$. We see that the degree of precision afforded by the two methods is about the same, and is amply sufficient for all practical purposes. But it still remains to be seen how far the TATLOCK method is available for the analysis of salts which are relatively poor in potash.

Analyses made with the view of deciding this question will be submitted presently; but we prefer to interpolate a duplicate analysis, by both methods, of an imitation of the double salt $\mathrm{MgK}_{2} \mathrm{~S}_{2} \mathrm{O}_{8}+6 \mathrm{H}_{2} \mathrm{O}$.

$0.5370 \mathrm{grm}$. of chloride of potassium was converted into neutral sulphate (obtained $0.6273 \mathrm{grm}$. equivalent, by calculation, to 0.5368 of $\mathrm{KCl}$ ); this was dissolved in water, and diluted to a known weight. Two portions of the solution were weighed out, each mixed with the calculated weights of a standard solution of $\mathrm{MgSO}_{4}$, prepared from pure oxide by solution in dilute sulphuric acid, and analysed, one by the TATLOCK method, the other by our form of the Finkener process.

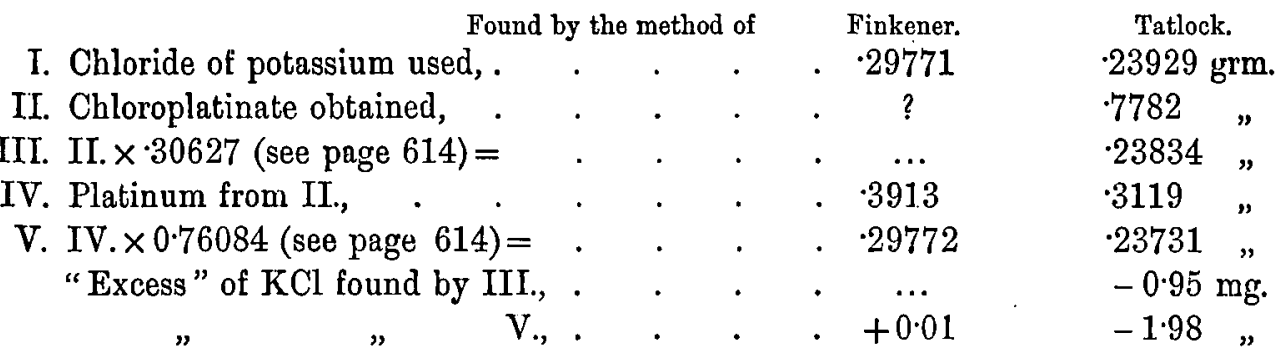

The TATLOCK method, as we see, gave a deficit of about $1 \mathrm{mg}$. of $\mathrm{KCl}$; but this, after all, is only $\frac{1}{240}$ th of the quantity to be determined, which suffices for all purposes; or, in other words, the TATLOCK method works well enough even with unmixed sulphates.

The substance analysed contained the equivalent of about 50.6 per cent. of $\mathrm{KCl}$.

We now pass to

$$
\text { A Set of Three Analyses of a " } 10 \text { per cent." Salt. }
$$

A standard solution was prepared, which represented a mixture containing (about) 79 of $\mathrm{NaCl}_{2} 6$ of $\mathrm{Na}_{2} \mathrm{SO}_{4}$, and 5 of $\mathrm{MgSO}_{4}$ in 90 parts. The 10 per cent. of $\mathrm{KCl}$ were weighed out specially for each analysis, as a standard solution.

Analysis (1)-Solution Finkenerised with twice the calculated weight of 
platinum, and enough (by calculation) of standard sulphuric acid to displace the chlorine of the chlorides. Mixture $\left(x \mathrm{R}_{2} \mathrm{SO}_{4}+\mathrm{PtCl}_{6} \mathrm{~K}_{2}\right)$ " recrystallised," platinum reduced out in the wet-way, and weighed.

Analysis (2)-Solution Finkenerised exactly as in (1); but recrystallisation omitted. From mixture $x \mathrm{R}_{2} \mathrm{SO}_{4}+\mathrm{PtCl}_{6} \mathrm{~K}_{2}$, the sulphates extracted by salammoniac; the residual chloroplatinate dissolved, the platinum reduced out, and weighed. The $\mathrm{KCl}$ contained in the filtrate beside $\mathrm{NH}_{4} \mathrm{Cl}$ recovered by evaporation and ignition, and weighed; then dissolved in water, wrought with $\mathrm{PtCl}_{6} \mathrm{H}_{2}$ (in FresenIUs' way), and the chloroplatinate weighed.

In Analysis (3), TatLock's method was applied in all strictness, except that the chloroplatinate received an extra washing with 6 drops of platinum solution.

Analysis (1)-Finkener's Method; Recrystallisation.

$\mathrm{A}=77 \cdot 22 \mathrm{mgs.} ; p=102 \cdot 2 ; p \times 0.76084=77 \cdot 76$;

Error $=+0.54 \mathrm{mg}$, or 0.7 per cent of $A$.

Analysis (2)-Finkener's Method; Sal-Ammoniac Form.

$\mathrm{A}=162 \cdot 44 \mathrm{mgs}$; $p=217 \cdot 4 ; p \times 0 \cdot 76084=165 \cdot 41$;

Excess over $\mathrm{A}=2.97 \mathrm{mgs} .{ }^{\prime}$ Crude $\mathrm{KCl}$ from filtrate (from $p$ ) $=166.9$; Excess over $A=4.5 \mathrm{mgs}$. Chloroplatinate from the crude chloride of potassium $=527.6 \mathrm{mgs}$; whence by multiplication with $0.30627=161.59$ mgs. of chloride of potassium; deficit against $\mathrm{A}=0.85 \mathrm{mg}$., or 0.52 per cent. Platinum out of the last chloroplatinate $=p^{\prime}=212 \cdot 0 ; p^{\prime} \times 0 \cdot 7608=161 \cdot 30$, which is less than A by $1 \cdot 14$ mgs., or $0 \cdot 70$ per cent.

\section{Analysis (3)-Tatlock's Method.}

$\mathrm{A}=65.44$ mgs. $; \mathrm{C}=209.2 ; \mathrm{C} \times 0.30627=64.07 \mathrm{~T} ; \mathrm{T}-\mathrm{A}=-1.37$, or $2 \cdot 1$ per cent of A. Platinum from $\mathrm{C}=84 \cdot 0=p . \quad p \times 0.7608=63.91=\mathrm{T}^{\prime}$. $\mathrm{T}^{\prime}-\mathrm{A}=-1.53$ mgs., or 2.3 per cent. of $\mathrm{A}$.

Analysis (2) did not do justice to its method, through unobserved causes, it is true. Yet the error in (1) or (2) did not rise beyond 0.7 per cent. of the small quantity to be determined. The TATLOCK method loses 2 per cent. of the chloride of potassium to be determined; i.e., it would report 9.8 instead of 10 per cent. We believe the line of the applicability of TATLOCK's process must be drawn at about the " 10 per cent." salt.

Three Analyses of synthetically prepared Sea-Water Salts.

(Average ocean-water salts contain $2 \cdot 11$ per cent. of potassium calculated as $\mathrm{KCl}$.) 
A kind of potash-free sea-water was made from pure materials : chloride of sodium, magnesia, and standard solutions of sulphuric and hydrochloric acids. For each analysis a volume corresponding to so and so much average oceanwater was measured out, and the exact weight of a standard solution of chloride of potassium added. The methods were exactly the same as those used in the preceding set.

Analysis (1)-Finkener's Method; "Recrystallisation."

Chloride of potassium used as such, . . . . . . . . . $=148.69 \mathrm{mgs}$.

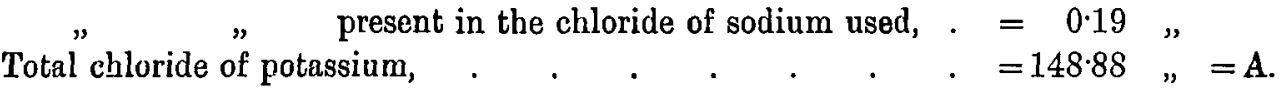

$p=198.3 ; p \times 0.76084,=150.87$; excess over $\mathrm{A}=1.99$ mgs., or 1.3 per cent. of $\mathrm{A}$ :-partly through compensation of errors.

The ether-alcohol washings, when worked up for potassium as usual, gave, $\left.\begin{array}{l}\text { The first washings } \mathrm{PtCl}_{6} \mathrm{~K}_{2}=0.36 \mathrm{mg} . \\ \text { The second " },=0.61, \text { " }\end{array}\right\}$ of $\mathrm{KCl}$.

This analysis was made by means of our present form of the Finkener process; the foreign bases were converted into sulphates only virtually, i.e., by adding the calculated volume of standard sulphuric acid, \&c.

In the "Challenger" analyses, the first step always was to actually convert all the bases into neutral sulphates, which probably ensures greater constancy in the results.

Analysis (2)-Finkener's Method; Sal-Ammoniac Form.

Total chloride of potassium operated upon (including that of the $\mathrm{NaCl}$ ), $=\mathrm{A}=148.59 \mathrm{mgs}$.

Platinum from the chloroplatinate $\left(+x \mathrm{NH}_{4} \mathrm{Cl}\right),=202 \cdot 1=p . \quad p \times 0 \cdot 76084$, $=153.77$; excess over $\mathrm{A}=5.18$ mgs., or 3.3 per cent of $\mathrm{A}$. Crude $\mathrm{KCl}$ (from filtrate from platinum), $=146 \cdot 0$. Chloroplatinate from the same $=0.4642 \mathrm{grm}$. $=142 \cdot 17=$ " $a$ " mgs. of $\mathrm{KCl}$; this is less than $\mathrm{A}$ by $6.42 \mathrm{mgs}$, or 4.3 per cent. of $\mathrm{A}$.

$\mathrm{KCl}$ recovered (as ultimately $\mathrm{PtCl}_{6} \mathrm{~K}_{2}$ ) from sal-ammoniac liquors, $=6.54 \mathrm{mgs} .=\Delta$; from ether-alcohol washings $=0.46 \mathrm{mg} .=\delta . \quad$ By addition, $a+\Delta=148 \cdot 71 \mathrm{mgs} . \quad(a+\Delta)-\mathrm{A}=+0.12 \mathrm{mg}$., or 0.08 per cent. of $\mathrm{A}$.

Here again the sal-ammoniac process failed to do justice to itself ; in many similar cases we obtained better results, in the sense that far less potassium passed into the sal-ammoniac. The method, unfortunately, is somewhat capricious ; the chloroplatinate does not always stand the sal-ammoniac treatment equally well. To determine small quantities of potassium correctly, the sal-ammoniac liquors must be worked up; and in no case dares the crude chloride of

VOL. XXXIII. PART II. 
potassium be accepted as pure $\mathrm{KCl}$. Yet the sal-ammoniac form of the FINKENER method is invaluable, being the only method for extracting a small proportion of potassium from a mass of, e.g., soda-salts; only it must be wrought with great circumspection, and in its original form be only used as a mode of extracting the potassium, not as a method for its determination.

\section{Analysis (3)_Tatlock's Method.}

Chloride of potassium used $=\mathrm{A}=14 \cdot 22 \mathrm{mgs}$.

(We could not have used as much as in (1) or (2) without wasting an unreasonable amount of platinum solution.) $\mathrm{C}=41.0 ; \mathrm{C} \times 0.30627=12.56=\mathrm{T}$. $\mathrm{T}-\mathrm{A}=-1.66 \mathrm{mgs}$., or $11 \cdot 7$ per cent. of $\mathrm{A}$.

Platinum from $\mathrm{C}=16.9 \mathrm{mgs} .=12.86 \mathrm{mgs}$. of $\mathrm{KCl}$. Chloride of potassium recovered from the washings, and weighed, ultimately as $\mathrm{PtCl}_{6} \mathrm{~K}_{2}=2.92 \mathrm{mgs}$; hence $A_{0}=11 \cdot 30 . \quad T-A_{0}=+1.26$ mgs., or $11 \cdot 1$ per cent. of $A_{0}$.

With salt-mixtures like sea-water salts the TATLOCK method obviously loses its applicability. Nor was it ever intended for such mixtures.

\section{Experiments on Chloroplatinate of Rubidium.}

These experiments were planned at a very early stage of our investigation. They were suggested by the obvious consideration that for the synthetical determination of the weight-ratio $\mathrm{Pt}: 2 \mathrm{Cl}$ between the platinum and fixed chlorine in chloroplatinates, chloride of rubidium should be better adapted than the potassium-salt, because, while itself soluble in alcohol, its chloroplatinate is less soluble in water than chloroplatinate of potassium. For a similar reason, chloride of cæsium should be preferable to chloride of rubidium; but we shrank from the great expense which would have been involved in procuring the necessary supply of the rarer of the two rare alkalies.

Our raw material for the preparation of chloride of rubidum was a supply of "rubidium alum" from Trommsdorff in Erfurt. The alum was dissolved in hot water, and its rubidium precipitated by addition of chloroplatinic acid, the precipitate washed, reduced in the dry way with hydrogen, and the chloride of rubidium extracted with water. As it turned out to contain a very appreciable quantity of sulphate, it was redissolved, reconverted into chloroplatinate, and recovered from the latter by means of hydrogen. The salt thus obtained was contaminated with sulphuric acid, and two or three repetitions of the cycle of operations failed to eliminate this impurity quite completely. Going by the aspect of the chloride of barium precipitate, the last precipitation, indeed, seemed to have effected no improvement; we therefore evaporated the whole of our (last) chloride of rubidium to dryness, and thus obtained about $32 \mathrm{grms}$. 
of a salt, which, apart from that trace of sulphuric acid, seemed to be very pure. In its aqueous solution, sulphuretted hydrogen produced no change; sulphide of ammonium had no immediate effect, and, on long standing, only an almost invisible precipitate settled out. $0.5 \mathrm{grm}$. of the salt, when dissolved and mixed with iodide of potassium, gave no precipitate (absence of thallium). The spectrum-apparatus revealed no trace of potassium.

In order to, at the same time, determine the sulphuric acid, and rehearse a method for its removal, 5 grms. of the salt were dissolved, chloride of barium added, the precipitate allowed to settle, and weighed. It amounted to 6.5 mgs., indicating 0.045 per cent. of $\mathrm{SO}_{3}$ in the preparation.

From the (concentrated) filtrate, the rubidium was precipitated by addition of a slight excess of chloroplatinic acid, the chloroplatinate allowed to settle, and washed by decanting filtration, first with water, then with 50 per cent., and lastly with absolute, alcohol. The weight of the chloroplatinate, after a preliminary drying at $120^{\circ}$, amounted to $11 \cdot 187$ grms.

1.0094 grms. of this chloroplatinate were dissolved in hot water, in an Erlenmeyer flask, the platinum was reduced out by hydrogen, and filtered off, and the filtrate evaporated to dryness over a water-bath. The residue was dissolved in 50 c.c. of water, and separate portions examined. 10 c.c. when mixed with sulphide of ammonium, gave a mere trace of ( $\mathrm{FeS}$ and $\mathrm{Al}_{2} \mathrm{O}_{3}$ ?); 10 c.c. when Nesslerised, gave $0.08 \mathrm{mg}$. of $\mathrm{NH}_{3}$, corresponding to $0.4 \mathrm{mg}$. per $1 \mathrm{grm}$. of salt; 10 c.c. when mixed with chloride of barium, and other 10 c.c. when mixed with sulphuric acid, gave both slight clouds of sulphate of baryta. From the last two tests, it was clear that the application of chloride of barium to our stock of salt would have done little good, and we accordingly decided upon using the preparation as it was.

A portion of it was dehydrated in a platinum crucible without fusion, then fused very cautiously, and poured out into a platinum basin. 18.1321 grms. of such salt were dissolved in water to 300 c.c., and thus converted into 313.522 grms. of a standard solution, of which every $1 \mathrm{grm}$. contained $57 \cdot 834$ mgs. of salt. Three titrimetric determinations of the chlorine gave the following results :-

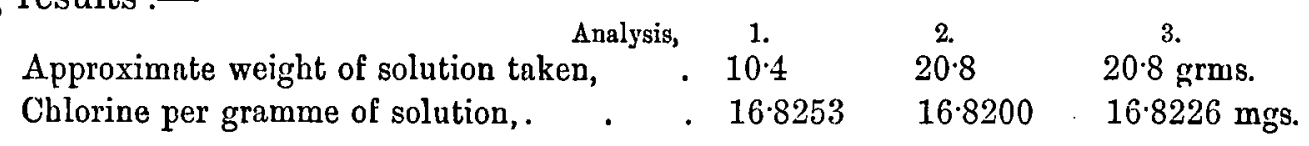

Mean $=16.8226$, corresponding to 29.087 per cent. of chlorine in the original salt.

Taking $\mathrm{Rb}=85.4$, and $\mathrm{Cs}=133.0$, the precentages of chlorine in the chlorides of the two metals are-

$$
\begin{array}{lllll} 
& & \text { In } & \text { RbCl. } & \text { CsCl. } \\
\text { Equal to, } & + & . & \cdot 29 \cdot 337 & 21.047
\end{array}
$$


Hence our "chloride of rubidium" contained

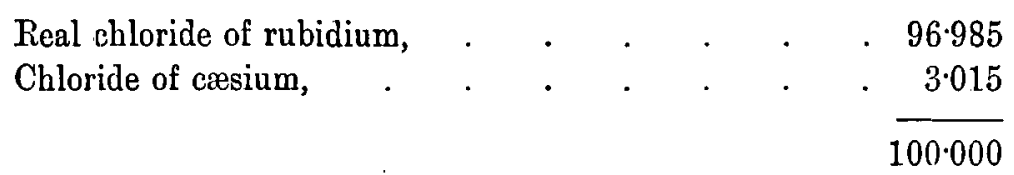

These 3 per cent. of cæsium chloride, although inconvenient, did not unfit the salt for our purpose; all we had to do was to base our calculations of " $\mathrm{Pt}$ " not upon the weight of alkyl-chloride present in the respective chloroplatinate, but upon the weight of the chlorine in that chloride.

The reason why we took such pains in standardising our rubidium solution, of course was, that we intended to rely chiefly on the synthetical data of our experiments; but unfortunately, these became almost valueless through a change in the strength of the rubidium solution, which was observed only after the greater part of the work had been completed; yet they were, and still are, of great use to ourselves as affording checks for the respective analyses. Only the latter are reported on in the following paragraphs:-

To begin with a case where no quantitative synthesis was attempted, let us give the results of an analysis of the chloroplatinate of rubidium referred to above, as having been obtained incidentally from about 5 grms. of salt.

$3 \cdot 2495$ grms. of this preparation (weighed after a preliminary drying at $120^{\circ}$ C.) were placed in a "Geissler tube," and dried systematically, first at $120^{\circ}$, then at $130^{\circ}$, and lastly at $150^{\circ} \mathrm{C}$. The weights recorded were as follows :-

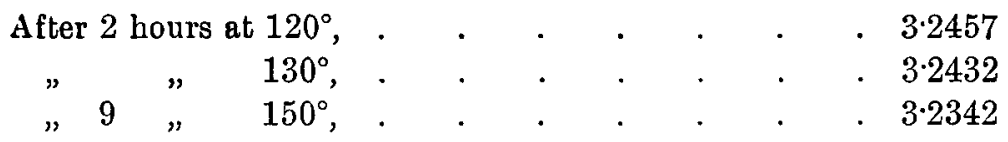

Even then the weight was not constant; yet the drying process was stopped, and 3.1970 grms. of the salt transferred to an Erlenmeyer flask, to be reduced with hydrogen in the wet way, and analysed in the way we had before applied to many specimens of the potassium salt.

The reduction set in very readily, but was very slow in coming to an end. It took in all about six days for its completion, although every morning the precipitate was broken up with a glass rod, to bring the hidden chloroplatinate to the surface. When at last the reduction seemed to be completed, the platinum was filtered off, and the filtrate divided gravimetrically for the determination of the fixed and of the total chlorine. Suspecting that the platinum might include some undecomposed chloroplatinate, the greater part was removed from the filter, and next dehydrated at a dull-red heat. It weighed 1.0454 grms.; after subsequent strong ignition this weight was 1.0452 grms. and after a succeeding strong ignition in hydrogen it was 1.0449 grms. Hence it appears that the proportion of undecomposed chloroplatinate 
in the "platinum" was at the worst extremely small. The total weight of platinum obtained amounted to 1.0923 grms.

For the isolation of the fixed chlorine, the respective portion of the solution was evaporated to dryness, the residue dried at $130^{\circ}$ for 2 hours, then dissolved in water, the solution re-evaporated, and the residue again dried at $130^{\circ}$ for $1 \frac{1}{2}$ hours. The solution of the thus dried salt was absolutely neutral to litmus.

The determinations of the chlorine were effected by the "Challenger" method as usual.

Summary of Results.

Found per $2 \mathrm{Cl}=70.91$ parts of fixed chlorine.

$\begin{array}{cccr}\begin{array}{c}\text { RCl by direct } \\ \text { weighing.* }\end{array} & \text { Platinum. } & \text { Loose Chlorine. } & \text { Substance. } \\ 244: 33 & 203 \cdot 39 & 144.71 & 595 \cdot 29 \\ & & =4.0816 \times \mathrm{Cl} . & \end{array}$

These results at the time surprised us very much ; but we have no difficulty now (after our later experience with the potassium salt) in explaining them. Part of the 203.39 parts of platinum must be assumed to be present as $\mathrm{PtX}_{6} \mathrm{H}_{2}$; the $\mathrm{X}_{6}$ including the $0.0816 \times \mathrm{Cl}$ of chlorine, besides the necessary amount of oxygen or hydroxyl. Taking $\mathrm{Pt}$ as 195.5 as it follows from our potassium experiments, we have for the composition of $595 \cdot 29$ of the chloroplatinate :-

$$
\begin{aligned}
& 2 \mathrm{RCl}, \quad \cdot \quad \cdot \quad \cdot \quad \cdot \quad \cdot \quad \cdot \quad \cdot 24433 \\
& 1.04036 \times \mathrm{Pt} \text {, : . . . . . . } 20339 \\
& \text { Loose chlorine, . . . . . . } 14471 \\
& \text { Hydroxyl, . . . . . . } 273=0.1606 \times \mathrm{OH} \text {. } \\
& \text { Hydrogen, . } \quad . \quad . \quad . \quad . \quad . \quad 0.08 \\
& 595 \cdot 24
\end{aligned}
$$

which agrees very well with the above $595 \cdot 29$ of substance analysed; only the closeness of the agreement is probably accidental, as the direct determination of the " $\mathrm{RCl}$ " was made only on a very small scale.*

We now pass to those synthetical experiments with standardised solutions, of which, unfortunately, only the analytical parts are worth publishing.

\section{Experiment I.}

40 c.e. of the rubidium solution were evaporated to 15 c.c., poured into 40.4 c.c. of standard platinum solution (1 c.c. $=49.5 \mathrm{mgs}$. of metal), and the

* A weighed portion of the filtrate from the platinum evaporated to dryness, and made neutral, as above explained, and weighed. Actual weight $=.2213 \mathrm{grm}$. for the fraction analysed. The salt was analysed, and found to contain 29.09 per cent. of chlorine, i.e., almost exactly as much as the original chloride of rubidium. 
mixture was allowed to stand over night. Next morning the liquor was decanted through a small filter, the precipitate washed four times with water $(10$ c.c, each time), and then twice with absolute alcohol. Absolute weight of platinum used $=1.9976$ grms.; platinum per $\mathrm{Cl}_{2}=70.91$ parts of fixed chlorine $=$ " $\mathrm{P} "$ $=201.8$ (nearly, the exact data need not be reproduced here). Total chloroplatinate produced $=5.78$ grms. It was dried at $130^{\circ} \mathrm{C}$. for 5 hours, and then divided into two parts, A and B. A served for a determination of the water by the direct method, described page $\tilde{5} 78$; water found $=0.862$ per cent. B was reduced with hydrogen in the wet way (which took about 10 days), and analysed as usual. Found, per $\mathrm{Cl}_{2}$ parts of fixed chlorine: alkyl chloride, by calculation from the percentage of chlorine in the original chloride of rubidium, $=243 \cdot 79$; platinum $=201.08$; loose chlorine $=4.0776 \times \mathrm{Cl}$; chloroplatinate (dried at $\left.130^{\circ}\right),=\mathrm{M}=593.81$; hence, assuming that $\mathrm{Pt}=195.5$, and that only so much of the platinum is there as $\mathrm{PtCl}_{6} \mathrm{R}_{2}$, we have for $\mathrm{M}$ parts-

$$
\begin{aligned}
& \text { Platinum, . . . . . . . . . . 201.08 }
\end{aligned}
$$

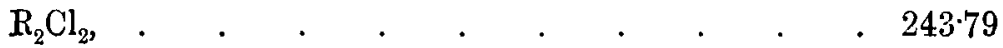

$$
\begin{aligned}
& \text { Other chlorine, . . . . . . . . . . . . } 14457 \\
& \text { Hydroxyl, . . . . . . . . . } \quad \text {. } 159
\end{aligned}
$$

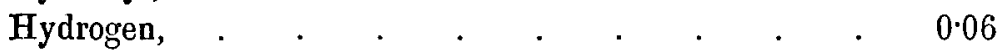

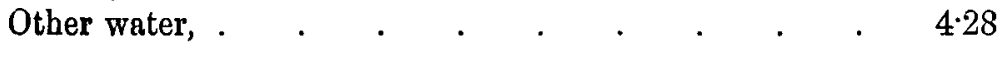

$$
\begin{aligned}
& \begin{array}{rrr}
\text { M., } \quad . \quad & 595.37 \\
\text { Excess over M., . } \quad=\frac{59.81}{1.56}
\end{array}
\end{aligned}
$$

\section{Experiment II.}

Conducted pretty much like Experiment I. Platinum used =1.48 grms.; $\mathrm{P}=299$. Chloroplatinate dried at $130^{\circ} \mathrm{C}$. Water not determined. As the reduction again progressed very slowly, the determination of the total chlorine was omitted. Found per $\mathrm{Cl}_{2}=70.91$ parts of fixed chlorine, $\mathrm{M}=583.60$; platinum $=196 \cdot 21$ only, although such a large excess of platinum solution had been employed for the production of the chloroplatinate! Just as in the case of the chloroplatinate of potassium, the presence of a large excess of chloroplatinic acid seems to prevent precipitation of surplus platinum as $\mathrm{Pt}(\mathrm{OH})_{6} \mathrm{H}_{2}$; the basic salt, which would otherwise have clung to the precipitate, passes into solution. Assuming that the loose chlorine in this case was double of the fixed, we have, for the composition of $M$ parts of chloroplatinate-

$$
\begin{aligned}
& \mathrm{R}_{2} \mathrm{Cl}_{2} \text {, . . . . . . . . . } 24379 \text { (calculated). } \\
& \mathrm{Cl}_{4}, \quad . \quad . \quad . \quad . \quad . \quad . \quad .141 \cdot 82 \\
& \text { Platinum, . . . . . . . . } 19621 \\
& \text { Water (?), . } \quad . \quad . \quad . \quad . \quad . \quad . \quad 179 \\
& 583.61
\end{aligned}
$$


CRITICAL EXPERIMENTS FOR DETERMINATION OF POTASSIUM, ETC. 623

\section{Experiment III.}

This experiment was carried on side by side of the preceding one, from which it differed in this, that the rubidium was kept in excess over the platinum solution, and that the latter was poured into the former. Platinum used $=0.987 \mathrm{grm} ; \mathrm{P}=132.6$. Chloroplatinate dried at $130^{\circ} \mathrm{C}$., but constancy of weight not insisted on. Determination of total chlorine and of water again omitted. Found per $\mathrm{Cl}_{2}=70.91$ parts of chlorine; $\mathrm{M}=602.43$; platinum $=204.94$; i.e., higher than ever before. [The synthesis gave 204.8 or 205.7 according to whether one or other of two limit values were adopted for the uncertain titre of the rubidium solution.]

\section{Experiment IV.}

Here again an excess of rubidium was used, and the chloroplatinic acid poured into the $\mathrm{RbCl}$. Precipitate treated, and dried at $130^{\circ} \mathrm{C}$., as before. For its analysis, however, it was reduced by hydrogen in the dry way. Platinum used $=0.991 \mathrm{grm}$. $\mathrm{P}=133.8$. Found per $\mathrm{Cl}_{2}$ parts of fixed chlorine, $\mathrm{M}=611.08$; platinum $=206.89$.

\section{Experiment V.}

A parallel experiment to IV., from which it differed only in this that an excess of platinum solution was started with, and the rubidium chloride poured into it. Platinum used $=1.4861$ grms.; $\mathrm{P}=301$. Found, per $\mathrm{Cl}_{2}$ parts of fixed chlorine, platinum $=198.19 ; \mathrm{M}$ not determined exactly. Here again, as in Experiment II., an excess of platinum used in the preparation of the chloroplatinate prevented, to some extent, precipitation of surplus platinum.

\section{Experiment VI.}

In this experiment a very large excess of chloride of rubidium was employed; and the reduction of the chloroplatinate effected in the wet way. To make sure of no chloroplatinate escaping reduction, it was divided into two parts (each equal to about 1 grm.), each part dissolved completely in hot water, and then submitted to the action of the hydrogen. To avoid loss of loose chlorine, the hydrogen entered from the Kipp's apparatus, and passed out from the Hasks through U-tubes, the bends of which were closed with a layer of water. The contents of these protection tubes, however, when tested after the experiment with nitrate of silver, were found to contain no trace of hydrochloric acid.

Platinum used $=0.6917$ grm.; $P=92 \cdot 9$. The chloroplatinate this time 
was dried only at $100^{\circ}$. Found per $\mathrm{Cl}_{2}$ parts of fixed chlorine :-M $=592 \cdot \tilde{6} \mathbf{5}$; platinum $=201.95$; loose chlorine $=4.0224 \times \mathrm{Cl}$. Hence, assuming $\mathrm{Pt}=195.5$, and that the surplus platinum $(201.95-195.50=6.45)$ is present as $\mathrm{PtX}_{6} \mathrm{H}_{2}$; we have, for the composition of M parts-

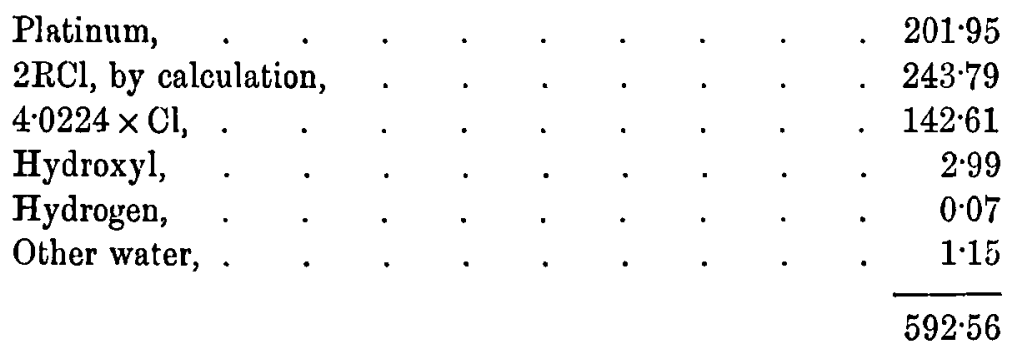

\section{Experiment VII.}

This experiment was carried out pretty much in the same way as Experiment VI. in the potassium series, and its object, like that of the latter, was to ascertain the composition of the unwashed chloroplatinate precipitate. 20 c.c. of the rubidium solution were weighed out, and poured into a tared bottle of 120 c c.'s capacity ; a known (predetermined) weight of platinum solution was then added, the bottle filled up to near the shoulder with water (total volume $=200$ c.c. about), the whole mixed, and allowed to stand over night. Platinum used $=0.969$ grm.; $P=195 \cdot 2$.

After determination of the exact weight of the whole, as much as possible of the clear liquor was sucked off into a tared Erlenmeyer flask, weighed, and analysed by reduction with hydrogen. To be able to determine the loose chlorine of the precipitate, the chloroplatinic acid solution was analysed immediately before the experiment, by reducing a known weight with hydrogen, weighing the platinum, and determining the chlorine in the filtrate; the small quantity of fixed chlorine in that solution being determined by itself, and allowed for. The uncertainty in the titre of the rubidium solution of course affected this experiment as well as it did the (unpublished) synthetical data of Experiments I. to VI; yet this uncertainty is not sufficient to invalidate the principal result, which was that (for every $\mathrm{Cl}_{2}$ parts of fixed chlorine) the platinum amounted to 205.48 parts, \pm say one unit; but even 204.5 would be a very high result. The loose chlorine amounted to $4 \cdot 151 \times \mathrm{Cl}$; a large excess over 4 .

\section{Experiment VIII.}

From the above experiments we concluded that our "chloroplatinates of rubidium" were not even normally constituted alkyl-chloroplatinates, i.e., mixtures of the composition $\mathrm{PtCl}_{6} \mathrm{R}_{2}$; but included hydrogen instead of part 
of the $\mathrm{R}_{2}$, and hydroxyl or oxygen instead of part of the $\mathrm{Cl}_{6}$; that, in short they were mixtures of the general composition $\mathrm{PtCl}_{(6-x)}(\mathrm{OH})_{x}+\left(\mathrm{R}_{2-y} \mathrm{H}_{y}\right)$, and we deemed it worth while to try and reduce the $y$ to nil by using chloroplatinate of sodium as a precipitant and the " $x$," by heating the precipitate in a current of dry hydrochloric acid.

In accordance therewith (St Petersburg) platinum solution equal to 1.100 grms. of metal was evaporated with $0.7616 \mathrm{grm}$. of chloride of sodium, i.e. $1 \cdot 15 \mathrm{Na}_{2} \mathrm{Cl}_{2}$ for 195 of platinum, to dryness on a water-bath, the residue redissolved in 20 c.c. of water, and added to the chloride of rubidium solution to produce a mixture containing about $0.68 \mathrm{grm}$. of surplus chloride of rubidium by calculation. The mixture was allowed to stand over night, and the precipitate then washed by decanting filtration, first with water, then with 50 per cent., and lastly with absolute, alcohol. The greater part of the dried precipitate was transferred to a large porcelain boat, and in it, within a combustion tube drawn out at the exit end, kept in a current of air, dried with oil of vitriol, at $120^{\circ} \mathrm{C}$., until its weight became constant, which took about six hours in all. The weight of the dried chloroplatinate was 2.7791 grms. The salt was then replaced in the combustion tube, a U-tube filled with glass beads moistened with vitriol attached to the exit end by means of a short india-rubber joint (see section on determination of water, page 578), and this joint next kept at $120^{\circ}$, in a current of dry air, until the U-tube ceased to gain weight; the U-tube consisted entirely of glass. The air was displaced by dry carbonic acid, the latter by hydrochloric aeid, and this gas allowed to act at $120^{\circ} \mathrm{C}$. for $3 \frac{1}{2}$ hours. The hydrochloric acid was then displaced by carbonic acid at $50^{\circ}$, and the latter by dry air, at the same temperature. The boat had gained $0.5 \mathrm{mg}$., the sulphuric acid U-tube $6.4 \mathrm{mgs}$. The contents of the latter, when diluted largely with water, and tested with nitrate of silver, gave a mere opalescence of chloride of silver. Hence the $6.4 \mathrm{mgs}$. of a gain were water, and, assuming that the substance lost water, and hydroxyl with formation of water $\left(\mathrm{HO}+\mathrm{HCl}=\mathrm{H}_{2} \mathrm{O}+\mathrm{Cl}\right)$, the water expelled amounted to $3.0 \mathrm{mgs}$, and the hydroxyl replaced by chlorine to $321 \mathrm{mgs}$. and the chlorine taken up in place of the latter to $6.7 \mathrm{mgs}$. The chloroplatinate was kept over solid caustic soda for a night, to remove any adhering $\mathrm{HCl}$, but it suffered no loss of weight. For its analysis, it was dissolved completely in hot water, reduced by hydrogen, \&c. As there was not sufficient material for duplicate analyses, the total and the fixed chlorine were each determined twice in the same quantity of solution, viz., gravimetrically, and again by determining the weight of unprecipitated silver titrimetrically. The results reduced to $2 \mathrm{Cl}$ parts of fixed chlorine were as follows :-

$\mathrm{M}=581.37$; platinum $=194.35 ;$ loose chlorine $=139 \cdot 81,=3.9434 \times \mathrm{Cl}$. Hence we have for the composition of M parts-

VOL, XXXIII. PART II. 


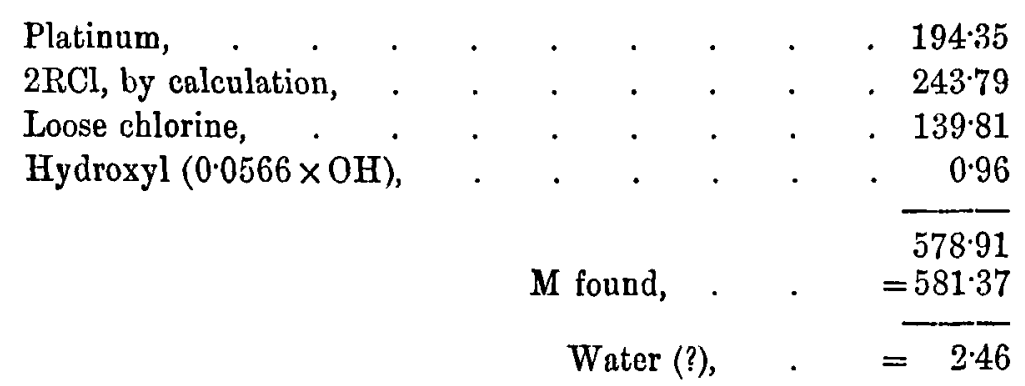

The $2 \mathrm{RCl}$, however, may have included $\mathrm{NaCl}$, which would make the deficit still greater, or it may have contained relatively more chloride of cæsium than the original "chloride of rubidium," which would have the opposite effect. Taking the experiment as it stands, it would appear that $\mathrm{Pt}=194 \cdot 35$, i.e., a little less than SEuberT's value; but it would be absurd to draw this conclusion from this one isolated experiment. A more plausible hypothesis is that the chloroplatinate contained surplus alkyl-chloride, which of course depresses the value $\mathrm{Pt}$ as calculated from the fixed chlorine. Assuming that the chloroplatinate contained its full complement of chlorine, and reducing the results to $6 \times \mathrm{Cl}$ parts of total chlorine, we have $\mathrm{M}=586.90$; and for the composition of those M parts-

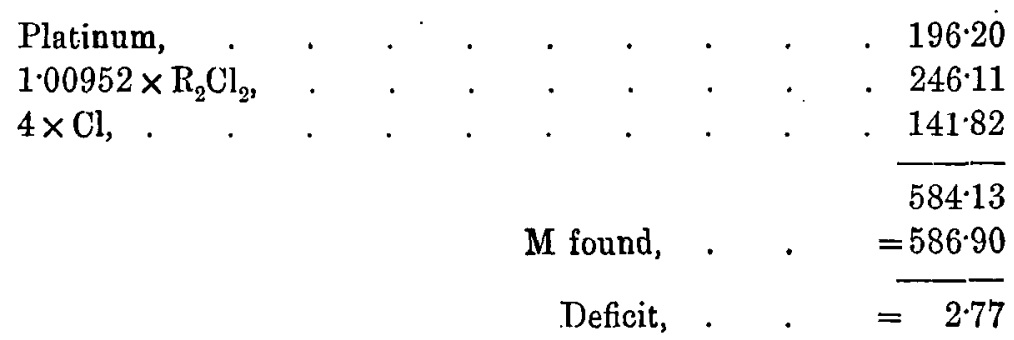

If we reduce to $\mathrm{Pt}=195 \cdot \dot{0}$, we have $\mathrm{M}=584 \cdot 82$; and for the composition of $\mathrm{M}$ parts,

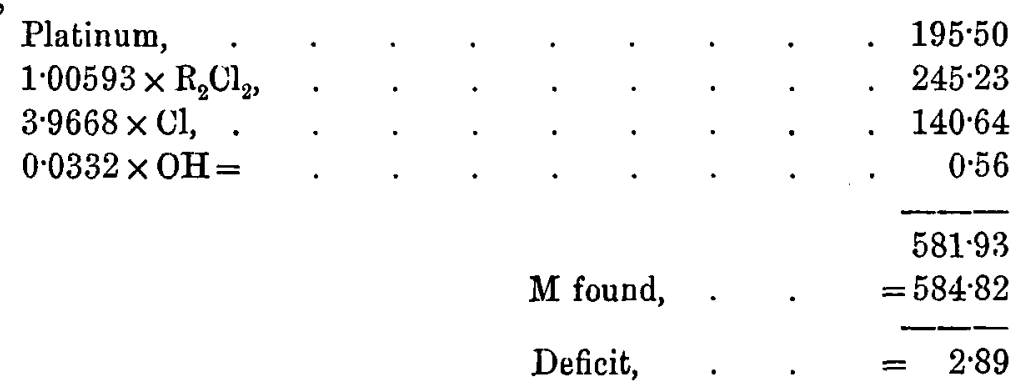

That the chloroplatinate analysed contained $0.00593 \times \mathrm{R}_{2} \mathrm{Cl}_{2}$ of free alkyl chloride is no improbable assumption.

None of our rubidium experiments afford the data for calculating even a limit value for the atomic weight of platinum; yet they are interesting, as showing that the tendency of chloroplatinate of potassium to carry down surplus platinum, in the form of chiefly hydroxide $\mathrm{Pt}(\mathrm{OH})_{6} \mathrm{H}_{2}$, when produced by precipitation of chloroplatinic acid with alkyl chloride, is greatly 
intensified in the rubidium salt; and in its case exhibits itself strongly even in the presence of a large excess of rubidium chloride.

Our last Experiment, VIII., may perhaps be referred to as showing that this irregularity can be avoided by using chloroplatinate of sodium as a precipitant, and (may we add ?) avoiding an excess of rubidium salt.

When we were engaged in these experiments we had not yet discovered the method of recrystallisation for the removal of surplus platinum, but an experiment made incidentally with one of our chloroplatinates, viz., the one whose analysis is quoted on page 621), proves that this chloroplatinate did contain surplus platinum. 2 grammes of it were boiled with 100 c.c. of water, the residue $(a)$ filtered off hot, the filtrate, which was strongly acid, cooled down, the precipitate formed by the cooling process filtered off, and the filtrate evaporated to dryness on a water-bath. The residue was treated with 8 c.c. of water, and the solution filtered. It gave no precipitate on addition of chloride of platinum, but a strong precipitate on addition of chloride of rubidium solution. The first residue $(a)$ when boiled with water, furnished a solution, which when evaporated, and (the residue) treated with 8 c.c. of water, gave a filtrate which produced slight precipitates with both reagents.

\section{Experiments on Chloroplatinate of Ammonium.}

For the preparation of the necessary supply of pure sal-ammoniac, we started from a kind of commercial liquor ammonice, of $0.88 \mathrm{sp}$. gr., which had been sold to us as having been prepared from volcanic sal-ammoniac. 300 c.c. of this liquor were mixed with 65 c.c. of specially prepared hydrochloric acid of 20 per cent., so as to neutralise about $\frac{1}{10}$ th of the volatile alkali, and the mixture heated, to drive out the still uncombined ammonia, which was passed into 360 c.c. of the hydrochloric acid. The resulting liquid was alkaline. It was evaporated in a Berlin basin over a water-bath, until a considerable quantity of sal-ammoniac had crystallised out, the residue allowed to cool, the magma of crystals collected on a funnel connected with a Bunsen pump, and washed with small instalments of 10 per cent. ammonia solution, made from the volcanic liquor, by distilling off ammonia from it, and passing it into water. The salt was then transferred to a shallow basin, and kept under a bell-jar in an atmosphere of (dilute) ammonia, over caustic soda sticks, which latter were renewed from time to time. After about a fortnight, the sal-ammoniac was transferred to another bell-jar, and under it, kept over oil of vitriol for 24 hours, to remove the ammonia adhering to the crystals. After this last operation, the sal-ammoniac was assumed to be pure, and was bottled up for the experiments.

To make sure of its purity, however, a known weight of the salt (about 1.6 grms.), was dissolved in water to a known weight, and the chlorine determined in 
two aliquot parts, both gravimetrically (with a known weight of silver used as nitrate) and titrimetrically, i.e., by determining the silver left unprecipitated, by means of our form of VOLHARD's method.

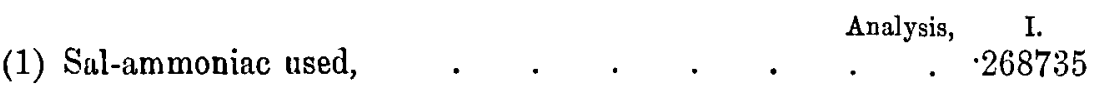

(2) Chlorine calculated from the weight of the $\mathrm{AgCl}$ obtained, -178059

(3) From the weight of the silver precipitated, . . . 178028

(4) Mean of (2) and (3), . . . . . . . . . 178044

(5) Substance analysed per 35.456 parts of chlorine, . $\quad 53.515 \quad 53.507$

Mean, . . . . . 53511

STAS' value is, . . . 53506

This sal-ammonic was used in the following experiments.

\section{Series.}

The general mode of operating was as follows :-

A known weight of the salt (about 0.54 grm.) weighed out as a standard solution, was mixed with a slight excess of chloroplatinic acid, the mixture evaporated to almost dryness over a water-bath, the residue treated with a mixture of equal volumes of absolute alcohol and absolute ether (both specially distilled with a little syrupy phosphoric acid, to eliminate any ammonia), and washed with such ether-alcohol until free of soluble chlorine. Only a small portion of the precipitate was allowed to get on the filter. To recover this small portion, it was allowed to dry in the air, then dried further at $100^{\circ}$, and what could not be removed mechanically, dissolved off with hot water; the solution was evaporated to dryness over a water-bath, the bulk of the precipitate added, and the whole kept in a drying-chamber at $100^{\circ}$ to $110^{\circ}$ until constant in weight. The chloroplatinate was then transferred to an Erlenmeyer flask, with some 300 c.c. of water, the platinum reduced out with hydrogen, filtered off, and weighed. The filtrate was diluted to a known weight, and a small portion utilised for a preliminary determination of the chlorine. A larger portion then served for the exact determination of the chlorine, by means of that combination of the gravimetric and VoLHARD's method, which had served for the analysis of the sal-ammoniac.

The ether-alcohol washings were collected and utilised for the determination of the small amount of ammonia which had escaped precipitation. These determinations, however, proved valueless, because we found out (only when all the work had been done) that the chloroplatinic acid used was contaminated appreciably with the nitroso-compound, $\mathrm{PtCl}_{6}(\mathrm{NO})_{2}$.

We therefore here satisfy ourselves with stating that the weights of unprecipitated sal-ammoniac appeared to amount to from 0.6 to $0.9 \mathrm{mg}$. per $0.54 \mathrm{grm}$. of sal-ammoniac operated upon.

Five experiments were made in this manner. The results are given in the 
following table, in which $\mathrm{P}$ stands for the weight of platinum used per $2 \mathrm{NH}_{4} \mathrm{Cl}$ parts of sal-ammoniac; A signifies the weight of sal-ammoniac used; $\mathrm{C}$, that of the chloroplatinate obtained; $p$, that of the platinum obtained from the chloroplatinate; $\mathrm{Pt}^{\prime}$ the weight of platinum present per $6 \times \mathrm{Cl}$ parts of total chlorine.

\begin{tabular}{|c|c|c|c|c|c|c|}
\hline $\mathrm{P}$ & Experiment, & I. & $\begin{array}{c}\text { II. } \\
4 \text { very } n\end{array}$ & $\begin{array}{c}\text { III. } \\
\text { throughou }\end{array}$ & IV. & v. \\
\hline$A: C$ & . & $\cdot 23873$ & .23970 & $(24139)$ & $\cdot 23948$ & $\cdot 23984$ \\
\hline A : $p$ & . & $\cdot 54464$ & .54658 & lost & $\cdot 54616$ & .54743 \\
\hline $\mathrm{Pt}^{\prime}$, & . & 196.90 & 196.59 & $\ldots$ & $1.96 \cdot 73$ & $196 \cdot 48$ \\
\hline$p: \mathrm{C}$ & . & $\cdot 43833$ & $\cdot 43854$ & $\ldots$ & -43847 & $\cdot 43812$ \\
\hline
\end{tabular}

Mean Values contrasted with those calculated from Seubert's $\mathrm{Pt}=194 \cdot 8$.

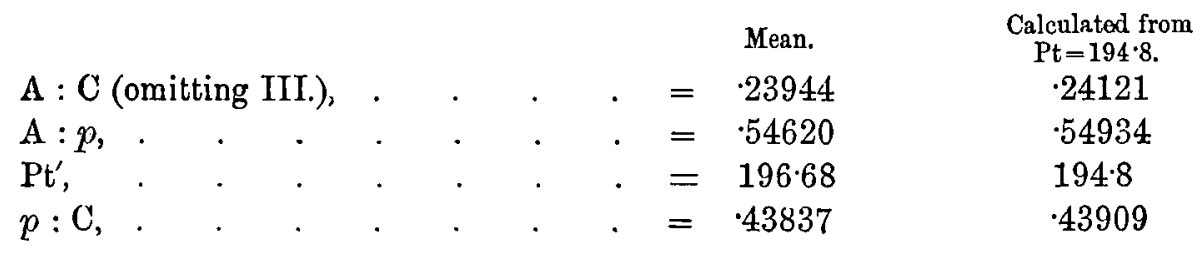

As the values $\mathrm{Pt}^{\prime}$, and more still those of $p: \mathrm{C}$, agree fairly; while those for $A: C$ and $A: p$ vary to an unpleasant extent, we thought that a variable part of the ammonia might have been lost in the evaporations, - the presence of nitroso-compound in the reagent had not been noticed yet,-and therefore tried the following experiment:-The same quantities of standard salammoniac and chloroplatinic acid solutions as has been used in Experiment V. were mixed in a retort connected with a Liebig's condenser, and distilled down in a current of air, while immersed in a steam-bath, and thus kept near $100^{\circ} \mathrm{C}$. This somewhat tedious operation was continued until only about 1 c.c. of liquid was left in the retort. In the distillate, after neutralisation with ammonia-free caustic soda, the ammonia was determined by NEssLER's colorimetric method. It amounted to only $0.01 \mathrm{mg}$. This tends to show that under the circumstances chloroplatinate of ammonium is not liable to dissociation (into $2 \mathrm{NH}_{3}$ and $\mathrm{PtCl}_{6} \mathrm{H}_{2}$ ); but then the ammonia, which otherwise would have escaped as such, may have been destroyed by the nitrosocompound.

\section{Series.}

The chloroplatinates obtained in the above experiments were obviously not of a constant composition, reducible to the general formula $\mathrm{PtCl}_{6}\left(\mathrm{NH}_{4}\right)_{2}$. Thinking that the irregularities were perhaps caused by the process of evaporation, we prepared two quantities of chloroplatinate of ammonium by mere precipitation in the cold. In one case (I.) the chloroplatinic acid was in excess, and the sal-ammoniac poured into it; in the other (II.), the sal- 
ammoniac solution was in excess, and the chloroplatinic acid poured into it. In either case, 3.00 grms. of platinum (as $\mathrm{PtCl}_{6} \mathrm{H}_{2}$ ) were operated upon, and the two solutions, before being mixed, attenuated by addition of quantities of water so adjusted that the total volume on both sides was 150 c.c. In both cases the mixture was allowed to stand over night. Next morning the clear liquid was decanted through a small filter; the precipitate was then washed by decanting filtration, first with small instalments of water until the soluble chlorine was reduced to a fraction of milligramme (by calculation), then with absolute alcohol until all the soluble chlorine was proved to be away (by testing with nitrate of silver). The precipitates were dried at $105^{\circ}$ to $110^{\circ}$ until constant in weight. Each of the two chloroplatinates was divided into two approximately equal parts, and each part analysed by itself; the platinum was reduced out, filtered off, and weighed; the filtrate divided into two aliquot parts, and in each the chlorine determined gravimetrically. VolHARD's method was not used in this case, as our stock of standard silver had become exhausted. All duplicate determinations made agreed very well with each other. In the following table we give the values, $\mathrm{C}: p ; \mathrm{Pt}^{\prime}=$ weight of platinum per $6 \times \mathrm{Cl}$ parts of total chlorine; $\mathrm{Pt}^{\prime \prime}=$ weight of platinum per $\mathrm{Cl}_{6}\left(\mathrm{NH}_{4}\right)_{2}$ $=248.84$ parts of non-platinum in the precipitate, also the approximate weight $\mathrm{P}$ of platinum employed per $2 \mathrm{NH}_{4} \mathrm{Cl}$ parts of sal-ammoniac.

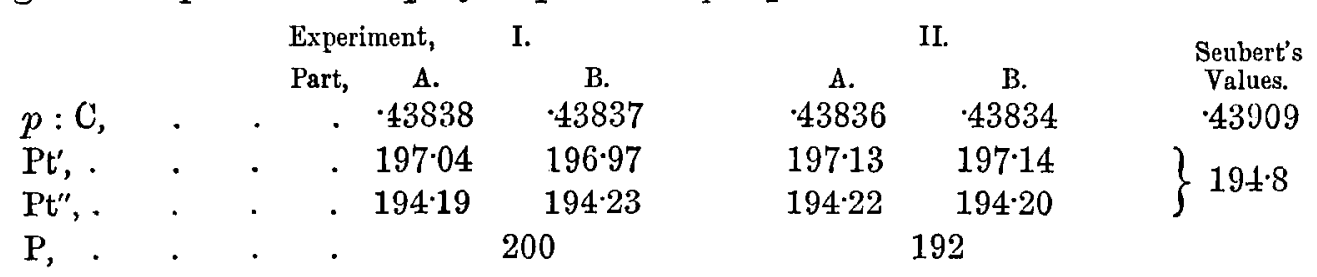

The most remarkable feature in these results is that the values $p: \mathrm{C}$, and $\mathrm{P}^{\prime}$, were the same in Experiment I. as in Experiment II. Clearly neither the mean of the values $\mathrm{Pt}^{\prime}$ nor that of the values $\mathrm{Pt}^{\prime \prime}$ can pretend to be a close approximation to the true $\mathrm{Pt}$. A reasonable hypothesis to account for the difference between $\mathrm{Pt}^{\prime}$ and $\mathrm{Pt}^{\prime \prime}$ is to assume that the chloroplatinate was a mixture of the composition $\mathrm{PtCl}_{6}\left(\mathrm{NH}_{4}\right)_{2}+x \mathrm{Pt}(\mathrm{OH})_{6} \mathrm{H}_{2}+y \mathrm{H}_{2} \mathrm{O}$. By calculating the four analyses as one, we found per $6 \times \mathrm{Cl}$ parts of chlorine-

$$
\begin{aligned}
& \text { Chloroplatinate, . . . . . }=\mathrm{M}=449.53 \\
& \text { Platinum, . . . . . . }=\mathrm{Pt}^{\prime}=197.06
\end{aligned}
$$

Assuming $6 \times \mathrm{Cl}$ to be associated with $2 \mathrm{NH}_{4} \mathrm{Cl}=107.01$, we have for the composition of the precipitate-

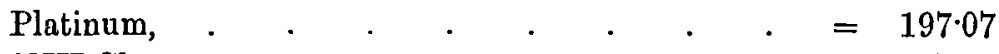

$$
\begin{aligned}
& 2 \mathrm{NH}_{4} \mathrm{Cl} \text {, . . . . . . . . . }=107.01 \\
& 4 \times \mathrm{Cl} \text {, . . . . . . . . }=141.82 \\
& \text { Unaccounted for, . . . . . . . . }=3.63 \\
& \mathrm{M}, \quad . \quad . \quad=\overline{449 \cdot 53}
\end{aligned}
$$


What value must we assign to $\mathrm{Pt}$, so that the $(\mathrm{OH})_{6} \mathrm{H}_{2}$, combined with the surplus platinum $(197.0 \overline{-}-\mathrm{Pt})$ equals these 3.63 parts ?

An easy calculation shows that we must take $\mathrm{Pt}=190 \cdot 4$, which, of course, is quite inadmissible, but part of these 3.63 parts may be water.

\section{Series.}

The Experiments reported on under I. and II. were made a good long time ago. Quite lately, when we were engaged in summing up results generally, it struck us that they had better be supplemented by new experiments made with a chloroplatinic acid prepared from metal by means of hydrochloric acid and chlorine gas, and we accordingly did so. The following three syntheses and analyses were made with the same kind of sal-ammoniac as had served for Series I. and II., and the general modus operandi was the same as in Series I. The ether-alcohol washings were subjected to distillation to remove the etheralcohol, the residue mixed with water, the platinum reduced out by hydrogen, and the mixture produced distilled with ammonia-free caustic soda, so as to concentrate the ammonia into a small quantity of hydrochloric solution. This solution was mixed with chloroplatinic acid, evaporated to dryness, and the residue washed with ether-alcohol, \&c., to recover the ammonia as chloroplatinate. The weight of sal-ammoniac operated upon in each experiment was about $0.814 \mathrm{grm}$., the weight of platinum used per $2 \mathrm{NH}_{4} \mathrm{Cl}$ was 227 parts, or about 1.15 times the quantity demanded by $\mathrm{Pt}=197$. The chloroplatinate precipitates were dried at $100^{\circ} \mathrm{C}$. until constant in weight.

The results are given in the following tables :-

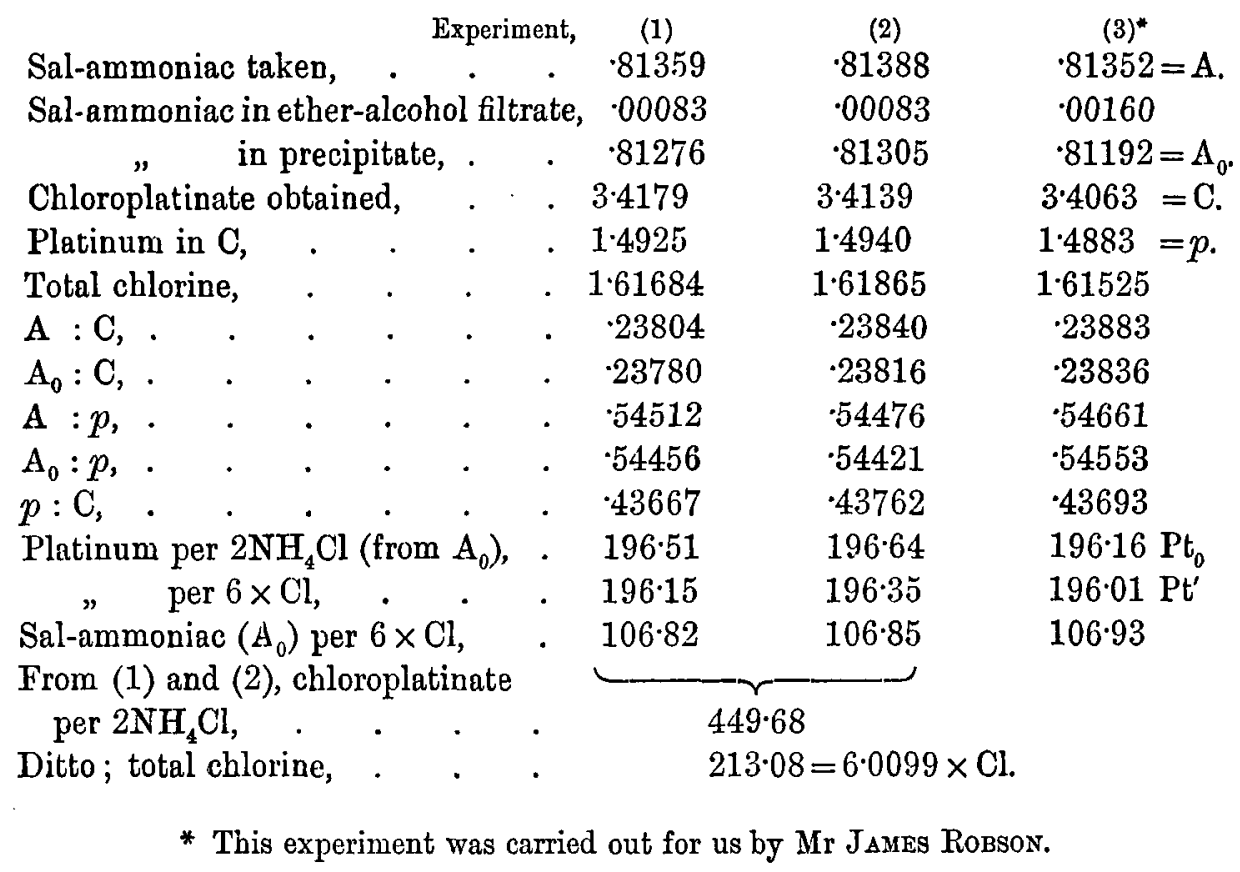


Hence, for the composition of the precipitate according to experiments (1) and (2)-

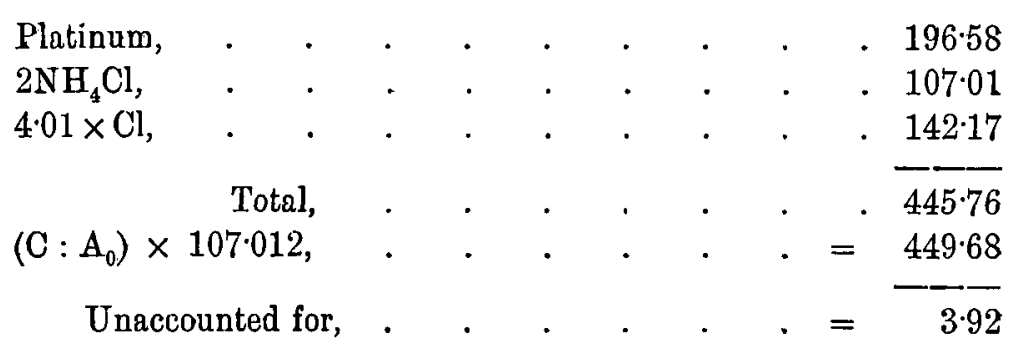

Probably part of the 196.58 parts of platinum is combined with the $0.01 \times \mathrm{Cl}$ and hydroxyl into $\mathrm{Pt}(\mathrm{Cl} \text { or } \mathrm{OH})_{6} \mathrm{H}_{2}$, which would account for part of the 3.92 of undetermined components.

These experiments again are compatible with any value for the atomic weight of platinum, from 196.58 down to about 190 .

Yet our experiments are of some value, because they show that if we weigh sal-amnoniac as chloroplatinate of ammonium, or as "platinum," we must not reduce our weighings to sal-ammoniac (or ammonia, or nitrogen) by means of factors calculated from the atomic weights concerned, but employ empirically determined factors.

According to our experiments, these factors are.

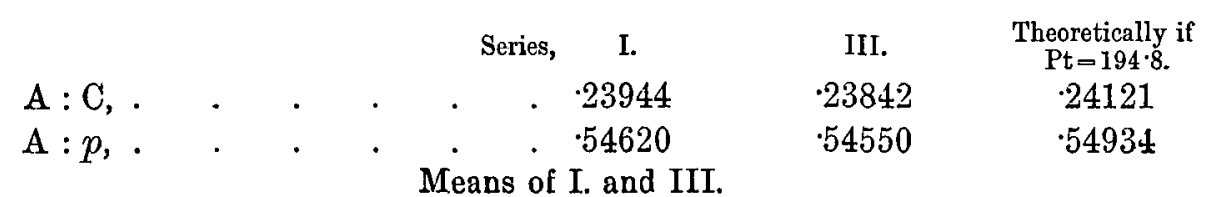

$$
\begin{aligned}
& \begin{array}{lllllll}
\mathrm{A}: \mathrm{C}, . & \cdot & \cdot & \cdot & \cdot & = & 23893 \\
\mathrm{~A}: p, & \cdot & \cdot & . & \cdot & = & 54585
\end{array}
\end{aligned}
$$

We are painfully aware that our individual determinations of these factors do not agree with one another as closely as we should wish. Yet we believe, when used as such in an analysis, they will afford a very fair approximation to the truth.

\section{Summary of Results.}

(1) The value $\mathrm{Pt}=194.8(\mathrm{O}=16)$, which Seubert deduced from his analyses of chloroplatinates, is too low; his own analyses, if properly interpreted, show that the true value is by a considerable fraction of a unit higher.

(2) From our own experiments on chloroplatinate of potassium, it appears that the true "Pt," though perhaps a shade below, lies close to, 195.5. This value falls in perfectly well with SeUbert's analyses; hence it, at present, is the most probable value.

(3) Taking " $\mathrm{Pt}$ " as the number which must be substituted for $\mathrm{Pt}$ in the 
CRITICAL EXPERIMENTS FOR DETERMINATION OF POTASSIUM, ETC. 633

calculation of the ratios $2 \mathrm{KCl}: \mathrm{Pt} ; 2 \mathrm{KCl}: \mathrm{PtCl}_{6} \mathrm{~K}_{2} ; 2 \mathrm{NH}_{4} \mathrm{Cl}: \mathrm{Pt}$, in order to obtain the correct factors for the reduction of analytically obtained chloroplatinate, or chloroplatinate-platinum, to chloride of potassium or ammonium, even our number 195.5 is too low ; 196 affords in general a closer approximation. But "Pt," taken in this sense, is no constant at all. Those factors must be determined directly by standard experiments. The results of our own standard experiments are given, and contrasted with theoretically calculated ratios, in the following table. The entries "Ta" refer to TatLock's method; the entries "F" to our own modification of FinkeneR's process as we used it for the analysis of the " $95,82,33 \cdot 3$ per cent." salt-mixtures; the entries " $N$ " to the customary platinum method for the determination of ammonia.

\begin{tabular}{|c|c|c|c|c|c|c|}
\hline \multirow{2}{*}{ Method. } & \multicolumn{4}{|c|}{ Theoretical Factors. } & \multirow{2}{*}{$\begin{array}{c}\text { D. \& M'A.'s } \\
\text { Empirical } \\
\text { Factors. }\end{array}$} & \multirow{2}{*}{ Note } \\
\hline & Symbols. & $\mathrm{Pt}=194 \cdot 8$ & $\begin{array}{l}\text { lues calculate } \\
\mathrm{Pt}=195 \cdot 5 \text {. }\end{array}$ & $\mathrm{Pt}=196$ & & \\
\hline Ta. & $2 \mathrm{KCl}: \mathrm{PtCl}_{8} \mathrm{~K}_{2}$ & ·30707 & $\cdot 30665$ & $\cdot 30633$ & $\cdot 30627$ & (1) \\
\hline Ta. & $2 \mathrm{KCl}: \mathrm{Pt}$ & $\cdot 76571$ & $\cdot 76307$ & $\cdot 76112$ & $\cdot 76016$ & (2) \\
\hline F. & $2 \mathrm{KCl}: \mathrm{Pt}$ & $\cdot 76571$ & $\cdot 76307$ & $\cdot 76112$ & $\cdot 76084$ & \\
\hline N. & $2\left(\mathrm{NH}_{4}\right) \mathrm{Cl}: \mathrm{PtCl}_{6}\left(\mathrm{NH}_{4}\right)_{2}$ & $\cdot 24123$ & $\cdot 24084$ & $\cdot 24057$ & $\cdot 2389$ & (3) \\
\hline N. & $2\left(\mathrm{NH}_{\triangleleft}\right) \mathrm{Cl}: \mathrm{Pt}$, & .54934 & $\cdot 54737$ & .54598 & 5459 & (4) \\
\hline \multicolumn{7}{|c|}{ Notes. } \\
\hline & $\begin{array}{l}\text { Refers to the chloride of po } \\
\text { Refers to the chloride of po } \\
\left.\text { and ( }{ }^{4}\right) \text { both refer to the sal-ar } \\
\text { cate. }\end{array}$ & tassium in & he substar & analysed. & pitate. & \\
\hline
\end{tabular}

